\title{
Boundary Element Analysis of Thick-Walled Spherical Vessels with Surface Cracks
}

by

\author{
Joshua Richard Armitage
}

\begin{abstract}
A Thesis submitted to
the Faculty of Graduate Studies and Research

in partial fulfillment of

the requirements for the degree of

Master of Applied Science
\end{abstract}

\author{
Ottawa-Carleton Institute for \\ Mechanical and Aerospace Engineering \\ Department of Mechanical and Aerospace Engineering \\ Carleton University \\ Ottawa, Ontario, Canada
}

September 2009

Copyright $($ C

2009 - Joshua Richard Armitage 


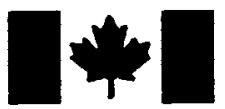

Library and Archives
Canada

Published Heritage Branch

395 Wellington Street Ottawa ON K1A ON4 Canada
Bibliothèque et

Archives Canada

Direction du

Patrimoine de l'édition

395 , rue Wellington

Ottawa ON K1A ON4

Canada
Your file Votre référence

ISBN: 978-0-494-60288-1

Our file Notre référence

ISBN: 978-0-494-60288-1

\section{NOTICE:}

The author has granted a nonexclusive license allowing Library and Archives Canada to reproduce, publish, archive, preserve, conserve, communicate to the public by telecommunication or on the Internet, loan, distribute and sell theses worldwide, for commercial or noncommercial purposes, in microform, paper, electronic and/or any other formats.

The author retains copyright ownership and moral rights in this thesis. Neither the thesis nor substantial extracts from it may be printed or otherwise reproduced without the author's permission.
AVIS:

L'auteur a accordé une licence non exclusive permettant à la Bibliothèque et Archives Canada de reproduire, publier, archiver, sauvegarder, conserver, transmettre au public par télécommunication ou par l'Internet, prêter, distribuer et vendre des thèses partout dans le monde, à des fins commerciales ou autres, sur support microforme, papier, électronique et/ou autres formats.

L'auteur conserve la propriété du droit d'auteur et des droits moraux qui protège cette thèse. $\mathrm{Ni}$ la thèse ni des extraits substantiels de celle-ci ne doivent être imprimés ou autrement reproduits sans son autorisation.
In compliance with the Canadian Privacy Act some supporting forms may have been removed from this thesis.

While these forms may be included in the document page count, their removal does not represent any loss of content from the thesis.
Conformément à la loi canadienne sur la protection de la vie privée, quelques formulaires secondaires ont été enlevés de cette thèse.

Bien que ces formulaires aient inclus dans la pagination, il n'y aura aucun contenu manquant. 


\section{ABSTRACT}

The boundary element method (BEM) is used for the elastic stress analysis of internally pressurized thick-walled spherical vessels with internal surface cracks. Spherical vessels with and without a radial crossbore are considered. The three-dimensional formulation of the BEM is first applied to the linear elastic fracture mechanics analysis of a spherical vessel without a crossbore with an internal surface crack. Normalized stress intensity factors are obtained for a number of geometric combinations, namely, spheres with radius ratios from 1.5 to 3 , crack depths from $20 \%$ to $80 \%$ of the vessel thickness, and crack aspect ratios from 1 to 0.6 . The normalized stress intensity factor around the periphery of an internal surface crack is observed to stay relatively constant until in proximity to the free surface. At this location, this quantity for small semi-circular cracks tend to gradually increase in magnitude, while for larger cracks with this form, the reverse is found to be true. This trend becomes more evident as the radius ratio of the sphere is increased. Decreasing the crack aspect ratio shows that semi-elliptical cracks exhibit an abrupt increase in the normalized stress intensity factor at the free surface. Polynomial influence coefficients are also obtained for all the cases, which permit stress intensity factors to be determined for any load case on the same crack geometry using the influence function method.

The stress analysis of an uncracked spherical vessel with a radial crossbore is also performed using the axisymmetric formulation of the BEM. Spheres with the same radius ratios as mentioned above are considered, and the circumferential stress factor variation is determined for a crossbore radius ranging from $10 \%$ to $25 \%$ of the internal radius of the sphere. The maximum circumferential stress factor typically occurs at the intersection of the internal surface and crossbore. However, for relatively large crossbores, the maximum value can occur at the exterior surface.

Surface cracks initiated at the crossbore are also investigated. A three-dimensional analysis is performed to obtain normalized stress intensity factors for a representative set of possible geometries. For this study, a radius ratio of 2 is considered with a crossbore radius ranging from $10 \%$ to $25 \%$ of the internal radius, and circular cracks spanning $20 \%$ to $80 \%$ of the wall thickness. For the set of cases treated, it is shown that when cracks 
emanate from the crossbore at the internal radius of the sphere, the maximum value of normalized stress intensity factor along the crack front occurs at this intersection. 


\section{Acknowledgments}

The author would like to acknowledge his thesis supervisor, Professor C.L. Tan, for his patience and guidance throughout this study, as well as his parents for all their support. 


\section{Table of Contents}

Page

List of Tables $\quad$ i

List of Figures $\quad$ vi

Nomenclature $\quad$ xii

1. Introduction 1

1.1 Previous Work 1

1.2 Proposed Solution Method 3

1.3 Outline 4

2. Review of the Boundary Element Method 5

2.1 Boundary Element Method Formulation 5

2.1.1 Numerical Solution of the Boundary Element Method 7

2.1.2 Numerical Example (A) - Spherical Vessel Subjected 9

to an Internal Pressure

2.1.3 Numerical Example (B) - Cylindrical Bar with a $\quad 10$

Spherical Cavity

2.2 Fracture Problems 11

$\begin{array}{ll}\text { 2.2.1 Crack-Front Boundary Elements } & 12\end{array}$

$\begin{array}{ll}\text { 2.2.2 Computation of Stress Intensity Factors } & 13\end{array}$

$\begin{array}{ll}2.3 \text { Numerical Examples - Fracture Problems } & 15\end{array}$

2.3.1 Elliptical Crack Embedded in an Infinite Body 15

2.3.2 Cylinder with a Semi-Elliptical Surface Crack 16

2.4 Influence Function Method for Obtaining Stress Intensity 17

Factors

2.4.1 Numerical Example - Cylinder with a Semi-Elliptical $\quad 18$

Surface Crack

$\begin{array}{ll}2.5 \text { Conclusions } & 19\end{array}$

3. Spherical Vessel with an Internal Surface Crack 41

3.1 Problem Definition 41

3.1.1 Numerical Model 42

$\begin{array}{ll}3.1 .2 \text { Results } & 42\end{array}$ 
3.2 Influence Coefficients $\quad 45$

3.2.1 Numerical Model $\quad 45$

3.2.2 Results 45

3.3 Conclusions 46

4. Spherical Vessel with a Crossbore $\quad 88$

4.1 Stress Analysis of a Spherical Vessel with a Crossbore $\quad 88$

4.1.1 Numerical Model $\quad 89$

4.1.2 Results $\quad 89$

4.2 Quarter-Circular Cracks at a Crossbore in a Spherical Vessel 91

4.2.1 Numerical Model 91

4.2.2 Results $\quad 92$

4.3 Conclusions 93

5. Conclusions 116

$\begin{array}{ll}\text { References } & 118\end{array}$

$\begin{array}{ll}\text { Appendix A } & 122\end{array}$

$\begin{array}{ll}\text { Appendix B } & 125\end{array}$ 


\section{List of Tables}

\section{Page}

Table 2.1. Stress concentration factors, $\sigma_{y} / \sigma$, for a cylindrical bar with a spherical cavity: $H / R_{b}=2$

Table 2.2. Normalized influence coefficients, $K_{l}^{(\mathrm{i})} / A_{i}(b / W)^{\mathrm{i}} \sqrt{ }(\pi b)$, for a thick-wall cylinder with a semi-elliptical surface crack: $k=2.5, b / a=0.8$, $b / W=0.4$

Table 3.1. Normalized stress intensity factors, $K_{l} / P \sqrt{ }(\pi b)$, for a spherical vessel subjected to an internal pressure with an internal surface crack, determined using the traction formula, $\mathrm{K}_{\mathrm{It}}$, and the displacement formulas, $\mathrm{K}_{\mathrm{Id}}: k=1.5, b / a=1$

Table 3.2. Normalized stress intensity factors, $K_{J} / P \sqrt{ }(\pi b)$, for a spherical vessel subjected to an internal pressure with an internal surface crack, determined using the traction formula, $\mathrm{K}_{\mathrm{l}}$, and the displacement formulas, $\mathrm{K}_{\mathrm{ld}}: k=1.5, b / a=0.8$

Table 3.3. Normalized stress intensity factors, $K_{l} / P \sqrt{ }(\pi b)$, for a spherical vessel subjected to an internal pressure with an internal surface crack, determined using the traction formula, $\mathrm{K}_{\mathrm{It}}$, and the displacement formulas, $\mathrm{K}_{\mathrm{ld}}: k=1.5, b / a=0.6$

Table 3.4. Normalized stress intensity factors, $K_{I} / P \sqrt{ }(\pi b)$, for a spherical vessel subjected to an internal pressure with an internal surface crack, determined using the traction formula, $\mathrm{K}_{\mathrm{It}}$, and the displacement formulas, $\mathrm{K}_{\mathrm{Id}}: k=2, b / a=1$ 
Table 3.5. Normalized stress intensity factors, $K_{I} / P \sqrt{ }(\pi b)$, for a spherical

vessel subjected to an internal pressure with an internal surface crack, determined using the traction formula, $\mathrm{K}_{\mathrm{It}}$, and the displacement formulas, $\mathrm{K}_{\mathrm{Id}}: k=2, b / a=0.8$

Table 3.6. Normalized stress intensity factors, $K_{I} / P \sqrt{ }(\pi b)$, for a spherical vessel subjected to an internal pressure with an internal surface crack, determined using the traction formula, $\mathrm{K}_{\mathrm{I}}$, and the displacement formulas, $\mathrm{K}_{\mathrm{Id}}: k=2, b / a=0.6$

Table 3.7. Normalized stress intensity factors, $K_{I} / P \sqrt{ }(\pi b)$, for a spherical vessel subjected to an internal pressure with an internal surface crack, determined using the traction formula, $\mathrm{K}_{\mathrm{I}}$, and the displacement formulas, $\mathrm{K}_{\mathrm{ld}}: k=2.5, b / a=1$

Table 3.8. Normalized stress intensity factors, $K_{I} / P \sqrt{ }(\pi b)$, for a spherical vessel subjected to an internal pressure with an internal surface crack, determined using the traction formula, $\mathrm{K}_{\mathrm{l}}$, and the displacement formulas, $\mathrm{K}_{\mathrm{Id}}: k=2.5, b / a=0.8$

Table 3.9. Normalized stress intensity factors, $K_{I} / P \sqrt{ }(\pi b)$, for a spherical vessel subjected to an internal pressure with an internal surface crack, determined using the traction formula, $\mathrm{K}_{\mathrm{lt}}$, and the displacement formulas, $\mathrm{K}_{\mathrm{Id}}: k=2.5, b / a=0.6$

Table 3.10. Normalized stress intensity factors, $K_{I} / P \sqrt{ }(\pi b)$, for a spherical vessel subjected to an internal pressure with an internal surface crack, determined using the traction formula, $\mathrm{K}_{\mathrm{It}}$, and the displacement formulas, $\mathrm{K}_{\mathrm{Id}}: k=3, b / a=1$ 
Table 3.11. Normalized stress intensity factors, $K_{I} / P \sqrt{ }(\pi b)$, for a spherical

vessel subjected to an internal pressure with an internal surface crack, determined using the traction formula, $\mathrm{K}_{\mathrm{lt}}$, and the displacement formulas, $\mathrm{K}_{\mathrm{Id}}: k=3, b / a=0.8$

Table 3.12. Normalized stress intensity factors, $K_{l} / P \sqrt{ }(\pi b)$, for a spherical vessel subjected to an internal pressure with an internal surface crack, determined using the traction formula, $\mathrm{K}_{\mathrm{It}}$, and the displacement formulas, $\mathrm{K}_{\mathrm{Id}}: k=3, b / a=0.6$

Table 3.13. Normalized stress intensity factors determined using influence coefficients, IC, and the direct method: $k=1.5, b / a=0.8, b / W=0.6$

Table 3.14. Normalized influence coefficients, $K_{I}^{(\mathrm{i})} / A_{i}(b / W)^{\mathrm{i}} \sqrt{ }(\pi b)$, for a spherical vessel subjected to an internal pressure with an internal surface crack: $k=1.5, b / a=1$

Table 3.15. Normalized influence coefficients, $K_{I}^{(i)} / A_{i}(b / W)^{i} \sqrt{ }(\pi b)$, for a 62 spherical vessel subjected to an internal pressure with an internal surface crack: $k=1.5, b / a=0.8$

Table 3.16. Normalized influence coefficients, $K_{l}^{(\mathrm{i})} / A_{i}(b / W)^{\mathrm{i}} \sqrt{ }(\pi b)$, for a 63 spherical vessel subjected to an internal pressure with an internal surface crack: $k=1.5, b / a=0.6$

Table 3.17. Normalized influence coefficients, $K_{I}^{(\mathrm{i})} / A_{i}(b / W)^{\mathrm{i}} \sqrt{ }(\pi b)$, for a 64 spherical vessel subjected to an internal pressure with an internal surface crack: $k=2, b / a=1$ 
Table 3.18. Normalized influence coefficients, $K_{I}^{(\mathrm{i})} / A_{i}(b / W)^{\mathrm{i}} \sqrt{ }(\pi b)$, for a spherical vessel subjected to an internal pressure with an internal surface crack: $k=2, b / a=0.8$

Table 3.19. Normalized influence coefficients, $K_{I}^{(\mathrm{i})} / A_{i}(b / W)^{\mathrm{i}} \sqrt{ }(\pi b)$, for a spherical vessel subjected to an internal pressure with an internal surface crack: $k=2, b / a=0.6$

Table 3.20. Normalized influence coefficients, $K_{l}^{(\mathrm{i})} / A_{i}(b / W)^{\mathrm{i}} \sqrt{ }(\pi b)$, for a spherical vessel subjected to an internal pressure with an internal surface crack: $k=2.5, b / a=1$

Table 3.21. Normalized influence coefficients, $K_{l}^{(\mathrm{i})} / A_{i}(b / W)^{\mathrm{i}} \sqrt{ }(\pi b)$, for a spherical vessel subjected to an internal pressure with an internal surface crack: $k=2.5, b / a=0.8$

Table 3.22. Normalized influence coefficients, $K_{I}^{(\mathrm{i})} / A_{i}(b / W)^{\mathrm{i}} \sqrt{ }(\pi b)$, for a spherical vessel subjected to an internal pressure with an internal surface crack: $k=2.5, b / a=0.6$

Table 3.23. Normalized influence coefficients, $K_{l}^{(\mathrm{i})} / A_{i}(b / W)^{\mathrm{i}} \sqrt{ }(\pi b)$, for a spherical vessel subjected to an internal pressure with an internal surface crack: $k=3, b / a=1$

Table 3.24. Normalized influence coefficients, $K_{I}^{(\mathrm{i})} / A_{i}(b / W)^{\mathrm{i}} \sqrt{ }(\pi b)$, for a spherical vessel subjected to an internal pressure with an internal surface crack: $k=3, b / a=0.8$

Table 3.25. Normalized influence coefficients, $K_{I}^{(\mathrm{i})} / A_{i}(b / W)^{\mathrm{i}} \sqrt{ }(\pi b)$, for a spherical vessel subjected to an internal pressure with an internal surface crack: $k=3, b / a=0.6$ 
Table 4.1. Circumferential stress factors, $K_{p}=\sigma_{\theta} P$, along the axial length

of a crossbore in a spherical vessel subjected to internal pressure: $k=1.5$

Table 4.2. Circumferential stress factors, $K_{p}=\sigma_{\theta} / P$, along the axial length

of a crossbore in a spherical vessel subjected to internal pressure: $k=2$

Table 4.3. Circumferential stress factors, $K_{p}=\sigma_{\theta} / P$, along the axial length

of a crossbore in a spherical vessel subjected to internal pressure: $k=2.5$

Table 4.4. Circumferential stress factors, $K_{p}=\sigma_{\theta} P$, along the axial length of a crossbore in a spherical vessel subjected to internal pressure: $k=3$

Table 4.5. Normalized stress intensity factors, $K_{I} / P \sqrt{ }(\pi b)$, determined using the traction formula, $K_{I t}$, and the displacement formulas, $K_{I d}$, around two circular cracks in the same plane at a crossbore in a spherical vessel subjected to internal pressure: $k=2, R_{S} / R_{I}=0.1$

Table 4.6. Normalized stress intensity factors, $K_{I} / P \sqrt{ }(\pi b)$, determined using the traction formula, $K_{I t}$, and the displacement formulas, $K_{I d}$, around two circular cracks in the same plane at a crossbore in a spherical vessel subjected to internal pressure: $k=2, R_{s} / R_{l}=0.15$

Table 4.7. Normalized stress intensity factors, $K_{I} / P \sqrt{ }(\pi b)$, determined using the traction formula, $K_{I t}$, and the displacement formulas, $K_{I d}$, around two circular cracks in the same plane at a crossbore in a spherical vessel subjected to internal pressure: $k=2, R_{s} / R_{l}=0.2$

Table 4.8. Normalized stress intensity factors, $K_{I} / P \sqrt{ }(\pi b)$, determined using the traction formula, $K_{I I}$, and the displacement formulas, $K_{I d}$, around two circular cracks in the same plane at a crossbore in a spherical vessel subjected to internal pressure: $k=2, R_{s} / R_{l}=0.25$ 


\section{List of Figures}

Page

Figure 2.1. Quadratic isoparametric eight-node quadrilateral element for three-dimensional BEM analysis

Figure 2.2. Quadratic isoparametric six-node triangular element for threedimensional BEM analysis

Figure 2.3. Quadratic isoparametric three-node line element for axisymmetric BEM analysis

Figure 2.4. Three-dimensional BEM mesh for a spherical vessel: (a) $k=$ 1.5 ; (b) $k=3$

Figure 2.5. Axisymmetric BEM mesh for a spherical vessel: (a) $k=1.5$;

(b) $k=3.0$

Figure 2.6. Variation of the normalized hoop stress, $\sigma_{\theta} P$, across the wall of a spherical vessel subjected to internal pressure: $k=1.5$

Figure 2.7. Variation of the normalized hoop stress, $\sigma_{\theta} / P$, across the wall of a spherical vessel subjected to internal pressure: $k=3$

Figure 2.8. Cylindrical bar with a spherical cavity

Figure 2.9(a). Axisymmetric BEM mesh for a cylindrical bar with a spherical cavity: $H / R_{b}=2, R_{a} / R_{b}=0.2$

Figure 2.9(b). Axisymmetric FEM mesh for a cylindrical bar with a spherical cavity: $H / R_{b}=2, R_{a} / R_{b}=0.2$ 
Figure 2.10. Three-dimensional BEM mesh for a cylindrical bar with a spherical cavity: $H / R_{b}=2, R_{a} / R_{b}=0.2$

Figure 2.11. Crack deformation modes

Figure 2.12. Stresses near the crack tip

Figure 2.13. Crack element notation for determining stress intensity factors directly

Figure 2.14. Three-dimensional BEM mesh for an elliptical crack in an infinite body: $b / a=0.8$

Figure 2.15. Definition of the parameters for an elliptical crack in an infinite body

Figure 2.16. Variation of the stress intensity factor, $K_{l}$, around the periphery of an elliptical crack in an infinite body: $b / a=0.8$

Figure 2.17. Diametrical section of a thick-wall cylinder with a semielliptical crack

Figure 2.18. Three-dimensional BEM mesh for a thick-wall cylinder with a semi-elliptical crack: $k=2.5, b / a=0.8, b / W=0.4$

Figure 2.19. Variation of the normalized stress intensity factor, $K_{I} / P \sqrt{ }(\pi b)$, around the periphery of a semi-elliptical surface crack in a thick-walled cylinder: $\mathrm{k}=2.5, \mathrm{~b} / \mathrm{a}=0.8, \mathrm{~b} / \mathrm{W}=0.4$

Figure 2.20. Superposition for determining stress intensity factors 
Figure 2.21. Variation of the normalized stress intensity factor, $K_{I} / P \sqrt{ }(\pi b)$, around the periphery of a semi-elliptical surface crack in a thick-walled cylinder determined directly and with influence coefficients: $\mathrm{k}=2.5, \mathrm{~b} / \mathrm{a}=$ $0.8, \mathrm{~b} / \mathrm{W}=0.4$

Figure 3.1. Diametrical section of a spherical vessel with an internal surface crack

Figure 3.2. Three-dimensional BEM mesh for a spherical vessel with an internal surface crack: $\mathrm{k}=1.5, \mathrm{~b} / \mathrm{a}=0.8, \mathrm{~b} / \mathrm{W}=0.4$

Figure 3.3. Variation of the normalized stress intensity factor, $K_{I} / P \sqrt{ }(\pi b)$, around the periphery of an internal surface crack in a spherical vessel subjected to an internal pressure: $k=1.5, b / a=1$

Figure 3.4. Variation of the normalized stress intensity factor, $K_{I} / P \sqrt{ }(\pi b)$, around the periphery of an internal surface crack in a spherical vessel subjected to an internal pressure: $k=2, b / a=1$

Figure 3.5. Variation of the normalized stress intensity factor, $K_{I} / P \sqrt{ }(\pi b)$, around the periphery of an internal surface crack in a spherical vessel subjected to an internal pressure: $k=2.5, b / a=1$

Figure 3.6. Variation of the normalized stress intensity factor, $K_{I} / P \sqrt{ }(\pi b)$, around the periphery of an internal surface crack in a spherical vessel subjected to an internal pressure: $k=3, b / a=1$

Figure 3.7. Variation of the normalized stress intensity factor, $K_{l} / P \sqrt{ }(\pi b)$, around the periphery of an internal surface crack in a spherical vessel subjected to an internal pressure: $k=1.5, b / a=0.8$ 
Figure 3.8. Variation of the normalized stress intensity factor, $K_{I} / P \sqrt{ }(\pi b)$, around the periphery of an internal surface crack in a spherical vessel subjected to an internal pressure: $k=1.5, b / a=0.6$

Figure 3.9. Variation of the normalized stress intensity factor, $K_{l} / P \sqrt{ }(\pi b)$, around the periphery of an internal surface crack in a spherical vessel subjected to an internal pressure: $k=2, b / a=0.8$

Figure 3.10. Variation of the normalized stress intensity factor, $K_{/} / P \sqrt{ }(\pi b)$, around the periphery of an internal surface crack in a spherical vessel subjected to an internal pressure: $k=2, b / a=0.6$

Figure 3.11. Variation of the normalized stress intensity factor, $K_{l} / P \sqrt{ }(\pi b)$, around the periphery of an internal surface crack in a spherical vessel subjected to an internal pressure: $k=2.5, b / a=0.8$

Figure 3.12. Variation of the normalized stress intensity factor, $K_{I} / P \sqrt{ }(\pi b)$, around the periphery of an internal surface crack in a spherical vessel subjected to an internal pressure: $k=2.5, b / a=0.6$

Figure 3.13. Variation of the normalized stress intensity factor, $K_{l} / P \sqrt{ }(\pi b)$, around the periphery of an internal surface crack in a spherical vessel subjected to an internal pressure: $k=3, b / a=0.8$

Figure 3.14. Variation of the normalized stress intensity factor, $K_{l} / P \sqrt{ }(\pi b)$, around the periphery of an internal surface crack in a spherical vessel subjected to an internal pressure: $k=3, b / a=0.6$ 
Figure 3.15. Variation of the normalized stress intensity factor, $K_{l} / P \sqrt{ }(\pi b)$, around the periphery of an internal surface crack in a thick-walled cylinder, a $90 \mathrm{o}$ degree section of curved tubing, and a thick-walled sphere subjected to an internal pressure: $k=2, b / a=0.8, b / W=0.4$

Figure 4.1. Diametrical cross-section of a spherical vessel with a crossbore

Figure 4.2. Axisymmetric BEM mesh of a spherical vessel with a crossbore: $k=2, R_{s} / R_{l}=0.15$

Figure 4.3. Variation of the circumferential stress factor, $K_{p}=\sigma_{\theta} / P$, along 104 the axial length of the crossbore in a pressurized spherical vessel determined using the axisymmetric BEM and FEM: $k=1.5, R_{s} / R_{l}=0.1$

Figure 4.4. Variation of the circumferential stress factor, $K_{p}=\sigma_{\theta} / P$, along the axial length of the crossbore in a pressurized spherical vessel: $k=1.5$

Figure 4.5. Variation of the circumferential stress factor, $K_{p}=\sigma_{\theta} P$, along the axial length of the crossbore in a pressurized spherical vessel: $k=2$

Figure 4.6. Variation of the circumferential stress factor, $K_{p}=\sigma_{d} P$, along the axial length of the crossbore in a pressurized spherical vessel: $k=2.5$

Figure 4.7. Variation of the circumferential stress factor, $K_{p}=\sigma_{\theta} P$, along 108 the axial length of the crossbore in a pressurized spherical vessel: $k=3$

Figure 4.8. Variation of the circumferential stress factor, $K_{p}=\sigma_{\theta} P$, along 109 the axial length of the crossbore in a pressurized cylinder and spherical vessel: $k=2, R_{s} / R_{l}=0.1$ 
Figure 4.9. Diametrical section of a spherical vessel with a crossbore containing two cracks on the same plane

Figure 4.10. Three-dimensional BEM mesh of a spherical vessel with a crossbore containing two circular cracks on the same plane: $k=2, R_{s} / R_{l}=$ $0.2, b / W=0.4$

Figure 4.11. Variation of the normalized stress intensity factor, $K_{l} / P \sqrt{ }(\pi b)$, around the periphery of circular cracks at a crossbore in a pressurized spherical vessel: $k=2, R_{S} / R_{I}=0.1$

Figure 4.12. Variation of the normalized stress intensity factor, $K_{l} / P \sqrt{ }(\pi b)$, around the periphery of circular cracks at a crossbore in a pressurized spherical vessel: $k=2, R_{S} / R_{I}=0.15$

Figure 4.13. Variation of the normalized stress intensity factor, $K_{L} / P \sqrt{ }(\pi b)$, around the periphery of circular cracks at a crossbore in a pressurized spherical vessel: $k=2, R_{s} / R_{I}=0.2$

Figure 4.14. Variation of the normalized stress intensity factor, $K_{/} / P \sqrt{ }(\pi b)$, around the periphery of circular cracks at a crossbore in a pressurized spherical vessel: $k=2, R_{s} / R_{I}=0.25$ 


\section{Nomenclature}

a Semi-major axis of the crack

$b \quad$ semi-minor axis of the crack

$k \quad$ Radius ratio $=R_{2} / R_{l}$

$l \quad$ Length of the crack element perpendicular to the crack front

$n_{i} \quad$ Unit outward normal

$p \quad$ Load point

$q \quad$ Field point

$r \quad$ Radial position; Perpendicular distance from the crack front

$r_{s} \quad$ Non-dimensional radial distance

$t_{j} \quad$ Boundary tractions

$u_{i} \quad$ Boundary displacements

$x_{i} \quad$ Cartesian coordinates

$z \quad$ Axial position

$A_{i} \quad$ Stress distribution coefficients

$C_{i j} \quad$ Geometry dependent coefficient

$G \quad$ Shear Modulus

$H \quad$ Half the length of a cylindrical bar

$J$ Jacobian of transformation

$K_{I} \quad$ Mode I stress intensity factor

$K_{J}^{\left({ }^{(}\right)} \quad$ Influence coefficients

$K_{p} \quad$ Circumferential stress factor

$N^{c} \quad$ Quadratic Shape Functions

$P \quad$ Internal pressure; Load point on the boundary

$Q \quad$ Field point on the boundary

$R_{I} \quad$ Internal radius

$R_{2} \quad$ External radius

$R_{a} \quad$ Spherical cavity radius

$R_{b} \quad$ Cylindrical bar radius

$R_{s} \quad$ Crossbore radius 
$T_{i j} \quad$ Traction solution for Kelvin's problem of a point force in an infinite body

$U_{i j} \quad$ Displacement solution for Kelvin's problem of a point force in an infinite body

$W \quad$ Thickness $=R_{2}-R_{l}$

$\delta_{i j} \quad$ Kronecker delta

$\Gamma \quad$ Boundary of a three-dimensional or two-dimensional domain

$\Omega \quad$ Three-dimensional or two dimensional domain

$\phi \quad$ Elliptical angle along the crack front

$v \quad$ Poisson's ratio

$\theta \quad$ Angle from the plane of the crack

$\sigma \quad$ Applied uniform Stress

$\sigma_{i j} \quad$ Stress Tensor

$\sigma_{\max } \quad$ Location of max stress

$\sigma_{r s} \quad$ Approximated polynomial of stress distribution

$\sigma_{\theta} \quad$ Hoop stress

$\xi \quad$ Local intrinsic coordinates 


\section{Chapter 1}

\section{Introduction}

Pressure vessels are used for many applications in the engineering industry. With advancements in technology, they are often required to withstand higher loads, thereby requiring them to have greater wall thicknesses. With increasing costs of raw materials, a more efficient use of material is highly desirable. The stresses in a spherical vessel due to an internal pressure are lower than those found in a cylindrical vessel of the same radius ratio. Thick-walled vessels are typically used in, for example, chemical processing industries and in the manufacture of synthetic diamonds. Failure of such high-pressure components would be catastrophic. This can occur when cracks are present in the

pressurized vessel. In order to pump a pressurized fluid into a spherical vessel, a crossbore needs to be introduced. This geometric discontinuity introduces stress concentrations which are usually the sites of crack initiation. The present study is concerned with the study of thick-walled internally pressurized spheres, with and without a crossbore, containing a surface crack or cracks. The primary objective is to obtain stress intensity factors for a relatively wide range of geometric parameters of the pressurized sphere.

\subsection{Previous Work}

Crack geometries, such as embedded and surface cracks in long prisms, were previously investigated by Blackburn and Hellen (1977) using the finite element method (FEM) incorporating special crack front elements. Also using the FEM, Atluri and Kathiresan (1979) determined stress intensity factors for thick-walled reactor pressure vessels with internal and external surface cracks of different depths and aspect ratios. The problem of a semi-elliptical surface crack in an internally pressurized thick-walled cylinder was also investigated by Tan and Fenner (1980) using the boundary element method (BEM). However, larger radius ratios and deeper cracks were considered, compared to the 
geometries analyzed by Atluri and Kathiresan (1979). Luchi and Poggialini (1983) developed special crack front elements that require no geometrical modifications. These elements have been incorporated in the BEM computer code used in the present study. Tan and Shim (1986) obtained influence coefficients for an internal surface crack in a thick-walled cylinder using the BEM. They were used to determine stress intensity factors for the same crack geometries due to thermal loads and the residual stresses caused by autofrettage. Also using the BEM, Plante (2008) determined the effects of bends in thick-walled tubing when cracks are present by obtaining stress intensity factors for a number of cases. Influence coefficients were obtained for the same crack geometries, which were used to investigate the existence of cracks in curved tubing subjected to autofrettage.

Numerical techniques have also been employed to determine the stress distribution in thick-walled cylinders with crossbores. Tan and Fenner (1980) considered cylinders containing one crossbore as well as two symmetrical crossbores. Side branches extending from crossbores were also investigated using the BEM by Fenner and Nadiri (1984). Tan (1986) also determined the stress re-distribution in a thick-walled cylinder due to the addition of a crossbore after autofrettage using the BEM and involving the superposition principle. Using the FEM, Masu (1997) and Makulsawatudom et al. (2004) determined stress concentration factors for offset and elliptical crossbores. Comlecki et al. (2007) performed similar analyses on relatively small radial circular crossbores.

The presence of cracks at the connection of a crossbore and the main bore of a thickwalled cylinder has also been investigated by Tan \& Fenner (1980) using the BEM. Two cracks were assumed to exist on the same plane, thereby adding a plane of symmetry to the problem. Stress intensity factors were obtained for different crossbore radii and crack aspect ratios.

Although much literature can be found on the topic of cracked pressurized thick-walled cylinders, the same cannot be said for thick-walled spherical vessels. Numerical and analytical procedures were determined by Parker \& Huang (2007) for stress and strain analysis of an autofrettaged spherical vessel. Adibi-Asi (2007) derived analytical expressions for the stress and strain in a sphere during the autofrettage process which were then used in the design for static and cyclic loading conditions. Perl (2007) 
developed an equivalent thermal load that simulates the residual stress distribution in an autofrettaged spherical vessel. It was then implemented using the FEM to accurately determine the stress distribution in an ideal autofrettage field, and one incorporating the Bauschinger effect. However, to the author's knowledge, fracture mechanics analyses of thick-walled spherical vessels with surface cracks have not previously been reported in the literature, and spherical vessels with cracks at stress concentrations such as crossbores have also not yet been investigated.

\subsection{Proposed Solution Method}

The Lamé solution for the stress fields in a plain spherical vessel subjected to an internal pressure is well known. However, the addition of a crossbore or surface defects makes the problem analytically intractable; recourse to numerical methods is usually necessary for such circumstances.

The boundary element method (BEM) is well established as an efficient numerical technique for the linear elastic stress analysis of problems with rapidly varying stresses, such as those near stress concentrations and cracks. With the BEM, only the boundary of the domain needs to be discretized into elements, as compared to the more commonly used finite element method (FEM) which requires modelling of the entire domain. This reduces the size of the numerical model, decreasing solution times and data preparation. Also, fewer elements are usually needed on the boundary for a given level of accuracy. If required, solutions at particular points in the interior of the domain can also be determined; in most problems however, including the present study, this is not necessary as the maximum stresses are usually on the surface of the domain. If problems contain planes of symmetry, advantage can be taken of this fact to reduce the size of the numerical problem, and solutions for stresses and displacements at points on these planes which are modelled can be obtained. The BEM will be used to carry out the numerical stress analysis of the spherical vessel in this study. 


\subsection{Outline}

A brief review of the BEM for three-dimensional and axisymmetric stress analyses will first be presented in the next chapter. Also in Chapter 2, linear elastic fracture mechanics is reviewed, along with the application of BEM to crack problems. Some examples are also provided to demonstrate the modelling techniques and the veracity of the solutions obtained. In addition, the influence function method is introduced in the next chapter; it allows stress intensity factors to be determined for the same crack geometry under an arbitrary load case.

In Chapter 3, a spherical vessel with an internal surface crack is investigated. The radius ratio of the sphere, crack aspect ratio, and relative crack depth are varied, and stress intensity factors are determined along the crack periphery for each geometric combination. Influence coefficients are also obtained for each case.

The stress distribution of an internally pressurized spherical vessel with a crossbore is analyzed in Chapter 4. Circumferential stress factors along the crossbore are obtained for different values of the two key radii, namely, the radius ratio of the sphere and the crossbore radius. Also in Chapter 4, cracks are introduced at the crossbore and stress intensity factors along the crack fronts are obtained for a representative set of the possible crack geometries. 


\section{Chapter 2}

\section{Review of the Boundary Element Method}

\subsection{Introduction}

The boundary element method (BEM) is a powerful tool for solving elastostatic problems, particularly ones with stress concentrations and cracks. Compared to other numerical techniques that require modelling the interior of the domain, such as the finite element method (FEM), fewer elements and nodes are required to achieve a given level of accuracy. This in turn decreases the size of the numerical problem, thereby reducing the time required to prepare and process the data. For the present study, threedimensional and axisymmetric BEM analyses were performed. A review of these two formulations is presented in the next section. As crack problems were also analyzed in the present study, it is useful to review the application of the BEM to fracture problems in this chapter. A more detailed description of the BEM and its applications can be found in the literature (see, e.g. Tan and Fenner (1979), and Becker (1992)). Also included in this chapter are some numerical examples, which help validate the selection of the BEM for this study, and show how the BEM would be applied to the problems mentioned above.

\subsection{BEM Formulation}

Let $\Gamma$ be the boundary of a three-dimensional domain, $\Omega$. Neglecting body forces, the boundary displacements, $u_{i}$, and tractions, $t_{j}$, can be related using the boundary integral equation, (see Cruse (1969)) expressed as

$$
C_{i j}(P) u_{j}(P)+\int_{\Gamma} T_{i j}(P, Q) u_{j}(Q) d \Gamma(Q)=\int_{\Gamma} U_{i j}(P, Q) t_{j}(Q) d \Gamma(Q)
$$


where $i, j=1,2,3$ in three-dimensions. However, if the problem is axisymmetric, $\Gamma$ becomes the boundary of the radial plane, that when rotated about the vertical axis $z$, will form the axisymmetric solid. The boundary integral equation (Equation 2.1) then becomes (see Tan and Selvadurai (1986))

$$
\begin{aligned}
C_{i j}(P) u_{j}(P) & +2 \pi \int_{\Gamma} T_{i j}(P, Q) u_{j}(Q) r d \Gamma(Q) \\
& =2 \pi \int_{\Gamma} U_{i j}(P, Q) t_{j}(Q) r d \Gamma(Q)
\end{aligned}
$$

where $i, j=r, z$ with $\mathrm{r}$ being the radial axis. $P$ and $Q$ are source and field points on the boundary; $C_{i j}$ is a coefficient that depends on boundary geometry at $P$, which may be written as

$$
C_{i j}(P)=\lim _{\varepsilon \rightarrow 0} \int_{\Gamma \varepsilon} T_{i j}(P, Q) d \Gamma
$$

If $P$ lies on a smooth surface,

$$
C_{i j}(P)=1 / 2 \delta_{i j}
$$

where $\delta_{i j}$ is the Kronecker delta. In Equations 2.1 and 2.2, $U_{i j}$ and $T_{i j}$ are the displacement and traction solutions, respectively, for Kelvin's problem of a point force in an infinite body. In three dimensions,

$$
\begin{gathered}
U_{i j}(p, q)=\frac{1}{16 \pi G(1-v) r}\left[(3-4 v) \delta_{i j}+r_{, i} r_{j}\right] \\
T_{i j}(p, q)=\frac{-(1-2 v)}{8 \pi(1-v) r^{2}}\left\{\frac{\partial r}{\partial n}\left[\delta_{i j}+\frac{3}{1-2 v} r_{i} r_{, j}\right]-r_{, i} n_{j}+r_{, j} n_{i}\right\}
\end{gathered}
$$

where $v$ is the Poisson's ratio, and $G$ is the shear modulus of the elastic body. Also, in Equations 2.5 and 2.6, $r$ is the distance between the field point $q$ and the load point $p$, written as 


$$
r=\sqrt{\left(x_{q i}-x_{p i}\right)\left(x_{q i}-x_{p i}\right)}
$$

Also

$$
r_{, i}=\frac{\partial r}{\partial x_{i}}=\frac{1}{r}\left(x_{q i}-x_{p i}\right)
$$

If $n_{i}$ is the unit outward normal vector, then

$$
\frac{\partial r}{\partial n}=\frac{\partial r}{\partial x_{i}} n_{i}=\frac{1}{r}\left(x_{q i}-x_{p i}\right) n_{i}
$$

The fundamental solutions, $U_{i j}$ and $T_{i j}$, for axisymmetry are much more complex (see e.g. Tan (1983)). They are presented in Appendix A.

The boundary integral equation (Equations 2.1 and 2.2) represents an integral constraint, relating the surface tractions and surface displacements. Since an exact analytical solution is not usually possible for most practical problems, a numerical solution of this equation needs to be performed. This is discussed in the next section.

\subsubsection{Numerical Solution of the BEM}

In the present study, the BEM involves modelling the surface of the numerical solution domain by discretizing it into quadratic isoparametric elements. In three-dimensions, eight-node quadrilateral and six-node triangular elements are used, as shown in Figures 2.1 and 2.2. However, when solving axisymmetric problems, only the radial plane of the axisymmetric body needs to be modelled using line elements. Three-noded quadratic isoparametric elements, as shown in Figure 2.3, are used here.

Quadratic shape functions in terms of local intrinsic coordinates, $\left(\xi_{1}, \xi_{2}\right)$ in threedimensions and $\xi$ for axisymmetry, are applied to the elements to represent the geometry and unknown tractions or displacements (see Tan and Fenner (1979) and Tan and Selvadurai (1986)), as shown below. 


$$
\begin{aligned}
& x_{i}(\xi)=N^{c}(\xi) x_{i}^{c} \\
& u_{i}(\xi)=N^{c}(\xi) u_{i}^{c} \\
& t_{i}(\xi)=N^{c}(\xi) t_{i}^{c}
\end{aligned}
$$

where

$$
\begin{gathered}
c=1,2, \ldots, 8 \text { for quadrilateral elements } \\
c=1,2, \ldots, 6 \text { for triangular elements } \\
c=1,2,3 \text { for axisymmetric line elements }
\end{gathered}
$$

and $N^{c}(\xi)$ are the quadratic shape functions, given in Appendix B. If there are $m$ elements and $q$ distinct nodes representing the surface, the three-dimensional boundary integral equation (Equation 2.1) becomes

$$
\begin{aligned}
C_{i j}\left(P^{a}\right) u_{j}\left(P^{a}\right) & +\sum_{b=1}^{m} \sum_{c=1}^{k} u_{j}\left(P^{d(b, c)}\right) \int_{\Gamma} T_{i j}\left(P^{a}, Q(\xi)\right) N^{c}(\xi) J(\xi) d \xi \\
& =\sum_{b=1}^{m} \sum_{c=1}^{k} t_{j}\left(P^{d(b, c)}\right) \int_{\Gamma} U_{i j}\left(P^{a}, Q(\xi)\right) N^{c}(\xi) J(\xi) d \xi
\end{aligned}
$$

and the axisymmetric boundary integral equation (Equation 2.2) becomes

$$
\begin{aligned}
C_{i j}\left(P^{a}\right) u_{j}\left(P^{a}\right) & +2 \pi \sum_{b=1}^{m} \sum_{c=1}^{k} u_{j}\left(P^{d(b, c)}\right) \int_{\Gamma} T_{i j}\left(P^{a}, Q(\xi)\right) N^{c}(\xi) J(\xi) d \xi \\
& =2 \pi \sum_{b=1}^{m} \sum_{c=1}^{k} t_{j}\left(P^{d(b, c)}\right) \int_{\Gamma} U_{i j}\left(P^{a}, Q(\xi)\right) N^{c}(\xi) J(\xi) d \xi
\end{aligned}
$$

where $P^{a}$ is the $a$ th node $(a=1, q), d(b, c)$ is the number of the $c$ th node of the $b$ th element, $J(\xi)$ is the Jacobian of transformation; $k=6$ for triangular elements, 8 for quadrilateral elements, and 3 for axisymmetric line elements. Equations 2.11 and 2.12 represent a set of algebraic equations in terms of the unknown displacements and tractions. In three- 
dimensions there are $6 q$ values of nodal displacements and tractions, half of which are known. Equation 2.11 represents the set of $3 q$ equations needed to solve for the other unknown displacements and tractions. When taking advantage of axisymmetry, there are $4 q$ values of nodal displacement and traction, half of which are known. Equation 2.12 then represents a set of $2 q$ equations for the other unknown displacements and tractions.

\subsubsection{Numerical Example (A) - Spherical Vessel Subject to Internal Pressure}

A spherical vessel with an internal pressure was analyzed using the three-dimensional program, BIE3D6, and the axisymmetric program, AXBIE2S to demonstrate how the BEM was applied in this study. The results were validated by comparing them to the theoretical solution (see Manning and Labrow (1974)). This problem was selected for demonstration purposes because the present study deals with an internally pressurized spherical vessel, but with surface cracks and/or cross-bores. Therefore this example helps demonstrate the efficiency of the BEM for the present study. Two spherical vessels were analyzed with radius ratios, $k=R_{2} / R_{l}$, equal to 1.5 and 3 , with $R_{1}$ and $R_{2}$ being the internal and external radii. Poisson's ratio was assumed to be 0.3 , and an internal pressure, $P$, was applied.

In three dimensions, a one-eighth model was used to represent the physical problem. It was constrained from displacement normal to the planes of symmetry. For the $k=1.5$ case, 36 elements and 104 nodes were used, as shown in Figure 2.4(a). For the $k=3$ case, 60 elements and 182 nodes were used, as shown in Figure 2.4(b).

For the axisymmetric solution, only half of the radial plane of the physical problem was modelled, as shown in Figure 2.5(a) for $k=1.5$, and Figure 2.5(b) for $k=3$. To account for symmetry, the bottom surface was constrained from displacement in the $\mathrm{z}$ - direction. In $\mathrm{BEM}$, it is not necessary to restrict rigid body motion and account for symmetry in the radial direction, as it is inherent in the numerical formulation. Also for the same reason, it is not necessary to model any surface that lies on the axis of rotational symmetry at $r=0$. The normalized hoop stress variations, $\sigma_{\theta} P$, across the thickness of the sphere determined using the three-dimensional and axisymmetric BEM are shown with the 
theoretical solution in Figures 2.6 and 2.7, respectively. Despite the relatively coarse meshes used, it can be seen that the two methods provide accurate representations of the theoretical solution. The discrepancy between the three-dimensional BEM results and the theoretical solution at the interior surface is less than $3.6 \%$, and less than $3.4 \%$ at the exterior surface. Comparing the axisymmetric BEM results to the theoretical solutions, a discrepancy of less than $1 \%$ at the interior and exterior surfaces was obtained. Here the axisymmetric solution is more accurate compared to the three-dimensional solution because more elements across the thickness of the sphere were used. Refining the axisymmetric mesh by simply adding one line element and two nodes considerably reduced the error in the BEM solution.

\subsubsection{Numerical Example (B) - Cylindrical Bar with Spherical Cavity}

Since the present study includes spherical vessels with cross-bores, it is necessary to demonstrate the efficiency of the BEM at analyzing stress concentrations. The problem of a cylindrical bar under tension with a spherical cavity was solved using the threedimensional and axisymmetric BEM. For comparison, the problem was also solved using the FEM software ANSYS 11. The results of the BEM and FEM were compared with the approximate analytical solution from Wang (2002). The cylindrical bar has an $H / R_{b}$ ratio equal to 2 , where $2 H$ is the bar's length and $R_{b}$ is the radius of the cylindrical bar, as shown in Figure 2.8. Three values of the spherical cavity radius, $R_{a}$, were considered with $R_{a} / R_{b}$ ratios equal to $0.2,0.4$, and 0.6 . Poisson's ratio was assumed to be 0.3 .

For the axisymmetric solution, half of the radial plane was modelled to take further advantage of the symmetry about the axial plane at $\mathrm{z}=0$. The mesh employed has 16 line elements and 33 nodes, as shown in Figure 2.9(a). The model was constrained from displacement in the normal direction on the plane of symmetry. A uniform tensile stress of $\sigma$ in the $\mathrm{z}$ direction was applied on the top face of the cylindrical bar. Note that near the spherical cavity the element size was reduced in order to accurately capture the stress concentration. Also, as shown in Figure 2.9(b), the mesh for the axisymmetric FEM solution required 104 elements and 361 nodes to obtain similar accuracy. 
The three-dimensional problem was represented using a one-eighth model with 103 elements and 307 nodes, as shown for the $R_{a} / R_{b}=0.2$ case in Figure 2.10. Displacement was constrained in the normal directions on all three planes of symmetry, and a uniform tensile stress $\sigma$ in the $\mathrm{x}_{3}$ direction was applied on top of the cylindrical bar.

The maximum stress for this problem occurs at the ring caused by the intersection of the cavity and the thinnest section of material, shown by $\sigma_{\max }$ in Figure 2.8. The stress concentration, $\sigma_{y} / \sigma$, at this location was recorded for each case. Table 2.1 shows both BEM solutions, together with the FEM and analytical results. Despite the relative coarseness of the BEM meshes, as compared to the FEM one, the results from the BEM solutions for the smaller cavity sizes are within $1.5 \%$ of the analytical solution. For the $R_{a} / R_{b}=0.6$ case, the discrepancies between the BEM results and the analytical solution are still within $4.5 \%$. Also, there is good agreement between both BEM results and FEM results, with discrepancies within $1 \%$.

\subsection{Fracture Problems}

In linear elastic fracture mechanics, the deformation of a crack can be characterized in terms of three modes, as shown in Figure 2.11. Mode I is the opening mode, mode II is the sliding mode, and mode III is the shear or tearing mode. For the purpose of this study, only mode I will be investigated, as it is the most common mode of fracture encountered in practice.

The displacement and stress fields in the crack region can be expressed by an infinite

power series $r^{1 / 2}, r, r^{3 / 2}, \ldots$ and $r^{-1 / 2}, r^{0}, r^{1 / 2}, \ldots$, respectively. Although in the vicinity of the crack front, only the first term of the series is retained, (see Luchi and Rizzuti (1987)) and these fields are characterized by the stress intensity factor $\mathrm{K}_{1}$. In mode I deformation, the stresses, $\sigma_{i j}$, near the crack front can be expressed as 


$$
\begin{aligned}
& \sigma_{11}=\frac{1}{\sqrt{2 \pi r}}\left[K_{I} \cos 1 / 2 \theta(1-\sin 1 / 2 \theta \sin 3 / 2 \theta)\right] \\
& \sigma_{22}=\frac{1}{\sqrt{2 \pi r}}\left[K_{I} \cos 1 / 2 \theta(1+\sin 1 / 2 \theta \sin 3 / 2 \theta)\right] \\
& \sigma_{33}=2 v\left(\sigma_{11}+\sigma_{22}\right) \\
& \sigma_{12}=\frac{1}{\sqrt{2 \pi r}}\left[K_{I} \sin 1 / 2 \theta \cos 1 / 2 \theta \cos 3 / 2 \theta\right] \\
& \sigma_{23}=\sigma_{31}=0
\end{aligned}
$$

and the displacements near the crack front are given by

$$
\begin{aligned}
& u_{1}=\frac{1+v}{4 E} \sqrt{\frac{2 r}{\pi}} K_{l}[(5-8 v) \cos 1 / 2 \theta-\cos 3 / 2 \theta] \\
& u_{2}=\frac{1+v}{4 E} \sqrt{\frac{2 r}{\pi}} K_{l}[(7-8 v) \sin 1 / 2 \theta-\sin 3 / 2 \theta] \\
& u_{3}=0
\end{aligned}
$$

In equations 2.14 and $2.15, r$ is the perpendicular distance from the crack front, and $\theta$ is the angle from the plane of the crack, as shown in Figure 2.12. It is evident from the equations that the displacements vary according to $\sqrt{r}$, and the stresses are singular, varying according to $1 / \sqrt{r}$. These behaviours are incorporated using special crack-front elements in the boundary element analysis. This will be discussed in the next section.

\subsubsection{Crack-Front Boundary Elements}

Special crack-front elements are required to accurately model the $\sqrt{r}$ displacement field and $1 / \sqrt{r}$ traction field near the crack front (Tanaka M. and Itoh H. (1987)).

In one type of crack-front element, the regular shape functions, as described in Section 2.1.1, are used with the only modification being the mid-side nodes adjacent to the crackfront being shifted to the quarter-point locations. This introduces the $\sqrt{r}$ displacement variation near the crack-front, but does not incorporate the $1 / \sqrt{r}$ traction singularity. 
However, this can be obtained by multiplying the shape functions by $l / \sqrt{r}$ (see e.g. Martinez and Dominguez (1984)).

The BEM code used for this study, BIE3D6, uses crack-front elements formulated by Luchi, and Poggialini (1983), which require no geometrical changes from the regular isoparametric quadrilateral elements. In order to represent the proper displacement and traction variations, special shape functions, different from those in Section 2.1.1, are applied to the crack-front elements. These shape functions are listed in Appendix B.

With the displacements and tractions properly defined, it is possible to accurately determine the stress intensity factor along the crack front, which governs crack propagation and stability.

\subsubsection{Computation of Stress Intensity Factors}

With the use of special crack-front elements, it is possible to derive formulas for determining the stress intensity factors directly using the computed tractions $t_{i}$, or displacements $u_{i}$ (Liu and Tan (1995)). These formulas are obtained by equating the tractions and displacements, as represented using the special shape functions, with the classical field solutions. With reference to Figure 2.13, the formulas are as follows. For corner nodes,

Traction Formula:

$$
K_{l}^{A}=\bar{t}_{1}^{A} \sqrt{\pi l}
$$

One-Point Displacement

Formula:

$$
K_{I}^{A}=\frac{2 G}{\kappa+1} \sqrt{\frac{\pi}{l}}\left(u_{1}^{D}-u_{1}^{\prime}\right)
$$

General Displacement

Formula:

$$
K_{l}^{A}=\frac{G(2+\sqrt{2})}{\kappa+1} \sqrt{\frac{\pi}{l}}\left[2\left(u_{1}^{D}-u_{1}^{I}\right)-\left(u_{1}^{E}-u_{1}^{J}\right)\right]
$$

where the superscripts refer to particular nodes as in Figure $2.13, l$ is the length of the crack element perpendicular to the crack front, and 


$$
\begin{aligned}
& \kappa=(3-4 v) \text { for plane strain } \\
& \kappa=\frac{3-v}{(1+v)} \text { for plane stress }
\end{aligned}
$$

Plane stress conditions are assumed at the free surface, but plane strain conditions are assumed at all other points on the crack front. For mid-side nodes, the formulas are as follows.

Traction Formula:

$$
K_{1}^{B}=\bar{t}_{1}^{-B} \sqrt{\pi l}
$$

One-Point Displacement

Formula:

$$
K_{I}^{B}=\frac{2 G}{\kappa+1} \sqrt{\frac{\pi}{2 l}}\left(u_{1}^{F}-u_{1}^{K}\right)
$$

General Displacement

Formula:

$$
\begin{aligned}
K_{I}^{B}= & \frac{G}{\kappa+1} \sqrt{\frac{\pi}{l}}\left[(2+\sqrt{2})\left(u_{1}^{D}+u_{1}^{H}-u_{1}^{I}-u^{M}\right)-(1+\sqrt{2})\right. \\
& \left.\times\left(u_{1}^{E}+u_{1}^{G}-u_{1}^{J}-u^{L}\right)+\sqrt{2}\left(u_{1}^{F}-u_{1}^{K}\right)\right]
\end{aligned}
$$

In equations 2.16 and $2.19, \bar{t}_{1}$ represents the traction coefficient at the representative crack front nodes. As can be seen from these equations, the traction formula is the easiest to use. It has also been shown to be the least mesh sensitive of the three (Liu and Tan (1995)). However, the advantage of using the traction formula together with one of the displacement formulas is that with a good mesh design, there is a good agreement between the stress intensity factors obtained. Therefore, to verify the results in the present study, all the stress intensity factors were determined using both computed tractions and displacements. Also worth noting is that the $1 / \sqrt{r}$ singularity does not exist at the free surface; the interpretation of the stress intensity factor at this location is thus somewhat ambiguous, and it should be used with caution. 


\subsection{Numerical Examples - Fracture Problems}

In order to validate the selection of the BEM for the crack problems in the present study, a number of test problems were analyzed. Two of these examples are discussed below.

\subsubsection{Elliptical Crack Embedded in an Infinite Body}

The problem of an elliptical crack embedded in an infinite body was one of the first examples analyzed, since the present study includes modelling of semi-elliptical cracks. The elliptical crack examined has an aspect ratio, $b / a=0.8$, where $b$ is the semi-minor axis and $a$ is the semi-major axis. Poisson's ratio was assumed to be 0.3 . Taking advantage of symmetry, only one-eighth of the problem needed to be modelled. The crack was modelled on the corner of a cube, ten times the size of the semi-major axis of the crack, to represent the infinite body. The mesh for this model is shown in Figure 2.14; it has 102 elements and 298 nodes. Displacement constraints were applied to all three planes of symmetry in their normal directions. This also prevented rigid body motion. A uniform stress was applied on the bottom face of the cube, normal to the plane of the crack, therefore causing mode I crack deformation.

The analytical solution to this example is (see e.g. Anderson (2005))

$$
K_{l}=\sigma \sqrt{\frac{\pi b}{Q a}}\left[\left(\sin ^{2} \phi+\left(\frac{b}{a}\right)^{2}\right) \cos ^{2} \phi\right]^{1 / 4}
$$

where $\sigma$ is the applied stress, $\phi$ is the elliptical angle in radians shown in Figure 2.15, and

$$
Q=1+1.464\left(\frac{b}{a}\right)^{1.65}
$$

Figure 2.16 shows the variation of the stress intensity factor, $K_{I}$, around the crack front determined using the traction formula, the general displacement formula, and the analytical solution. From this plot, it is evident that there is a very good agreement 
between both formulas and the analytical solution. The traction formula results are more accurate with discrepancies from the exact solution of within $1.2 \%$. The discrepancies between the displacement formula results and the analytical solution are between $2.25 \%$ and $3.8 \%$.

\subsubsection{Cylinder with a Semi-Elliptical Surface Crack}

Since the present study includes the analysis of spherical vessels with semi-elliptical surface cracks, a suitable example to test the BEM would be a thick-walled cylinder with a semi-elliptical surface crack, previously investigated by Tan and Shim (1986). The diametrical section of the problem is shown in Figure 2.17. The radius ratio of the cylinder is defined as $k=R_{2} / R_{l}$, the aspect ratio of the semi-elliptical crack is $b / a$, and the relative crack depth is $b / W$, where $W=R_{2}-R_{l}$. The case that was tested has the combination $k=2.5, b / a=0.8$, and $b / W=0.4$. Taking into account the symmetry of the crack, only one quarter of the problem was modelled. The mesh for this model, shown in Figure 2.18, has 119 elements and 337 nodes. A uniform pressure, $P$, was applied on the internal surface of the cylinder and the crack surface. Poisson's ratio was assumed to be 0.3. Displacement was constrained in the normal direction of the planes of symmetry, which prevented rigid body motion in the $\mathrm{x}_{1}$ and $\mathrm{x}_{3}$ directions, but not in the $\mathrm{x}_{2}$ direction. Therefore $\mathrm{x}_{2}$ displacement constraints were applied to the nodes on the vertical line created by the intersection of the external surface and the $x_{1}-x_{3}$ plane. Along this line, the tractions in the $\mathrm{x}_{2}$ direction are zero, and therefore this constraint did not restrict deformation, only rigid body motion. Also, the cylinder was constrained with plane strain end conditions.

The stress intensity factors obtained were normalized as $K_{I} / P \sqrt{ }(\pi b)$. Figure 2.19 shows the variation of the normalized stress intensity factor along the crack periphery as defined by angle $\phi$ in Figure 2.17. The results determined using the traction and general displacement formula are compared with those presented by Tan and Shim (1986). It can be seen that the results from both the traction formula and displacement formula are slightly higher than those obtained by Tan and Shim (1986), who had used the extrapolation technique to determine the stress intensity factors. Graphically, all the 
results show similar trends, and the discrepancies between the traction formula results and those of Tan and Shim (1986) are generally well within 6\%. Also, the discrepancies between the traction formula results and displacement formula results are within $4 \%$, except at the free surface, where as discussed earlier, values should be used with caution.

\subsection{Influence Function Method for Obtaining Stress Intensity Factors}

The influence function method is a simple technique that allows stress intensity factors for various load cases to be obtained for a given cracked geometry without performing new analyses of the whole problem (see Tan and Shim (1986)). Using the principle of superposition, the method involves the summation of the stress analysis of the uncracked component with the same external loads, and the cracked component with the loading only on the crack faces, as illustrated in Figure 2.20. The stress intensity factor can then be written as

$$
K_{l}=K_{l}^{()}+K_{l}^{(!)}
$$

The loads acting on the crack faces are simply equal and opposite to the loads that would exist on the crack face if the crack was not present. Since the stress intensity factor for the uncracked component is zero $\left(K_{I}^{\left({ }^{(}\right)}=0\right)$, the entire problem can be reduced to loading directly on the crack faces. Therefore,

$$
K_{i}=K_{i}^{(")}
$$

It is possible to determine the stress intensity factor in terms of polynomial influence coefficients, $K_{l}^{(i)}$, by

$$
K_{I}=K_{I}^{(0)}+K_{I}^{(1)}+K_{I}^{(2)}+K_{I}^{(3)}
$$


These influence coefficients are stress intensity factors of the problem with uncracked stress distributions applied directly to the crack faces. The distributions are: uniform ( $\sigma=$ $\left.-A_{0}\right)$, linear $\left(\sigma=-A_{l} r_{s}\right)$, quadratic $\left(\sigma=-A_{2} r_{s}{ }^{2}\right)$, and cubic $\left(\sigma=-A_{3} r_{s}{ }^{3}\right)$, where $r_{s}$ is a nondimensionalized distance, and $A_{i}$ are the coefficients of the distributions with units of stress. This assumes that the uncracked stress distribution on the prospective crack plane due to external loads can be expressed by a third degree polynomial as,

$$
\sigma_{r s}=A_{0}+A_{1} r_{s}+A_{2} r_{s}^{2}+A_{3} r_{s}^{3}
$$

The example in the following section shows how these influence functions are obtained, and how they are used to determine the stress intensity factors.

\subsubsection{Numerical Example - Cylinder with Semi-Elliptical Surface Crack}

The problem of the thick-walled cylinder with a semi-elliptical crack considered in Section 2.3.2 was used to illustrate the use of the influence function method. Using the same mesh and boundary conditions, four separate load cases were created. The computer code used, BIE3D6, is capable of handling multiple load cases, so the computational analysis of the four load cases only required one computer run. The load cases were uniform, linear, quadratic, and cubic tractions applied to the crack face, each normalized with $r_{s}$ and set to unity at $r=b$. The influence coefficients were determined, and then normalized with $A_{i}(b / W)^{i} \sqrt{ }(\pi b)$, as shown in Table 2.2 .

To obtain the uncracked stress distribution across the thickness of the cylinder, the Lame solution for a thick-walled cylinder under pressure was approximated as a cubic polynomial as follows

$$
\sigma_{r s}=P\left(1.3702-3.0401 r_{s}+3.7635 r_{s}^{2}-1.7545 r_{s}^{3}\right)
$$

The uncracked stress distribution coefficients in Equation 2.28 were multiplied by the appropriate normalized influence coefficients, and using Equation 2.26, they were then 
summed together to determine the stress intensity factors. As an example, the process is shown below for $\phi=0^{\circ}$, where $\phi$ is the angle defined in Figure 2.17.

$$
\begin{aligned}
& K_{I}^{(0)}=K_{I}^{(0)^{*}} A_{0} P(b / W)^{0} \sqrt{\pi b}=0.70321(1.3702 P) \sqrt{0.4 \pi}=1.0801 P \\
& K_{I}^{(1)}=K_{I}^{(1)^{*}} A_{1} P(b / W)^{1} \sqrt{\pi b}=0.49234(-3.0401 P)(0.4) \sqrt{0.4 \pi}=-0.6711 P \\
& K_{I}^{(2)}=K_{I}^{(2)^{*}} A_{2} P(b / W)^{2} \sqrt{\pi b}=0.40378(3.7635 P)\left(0.4^{2}\right) \sqrt{0.4 \pi}=0.2726 P \\
& K_{I}^{(3)}=K_{I}^{(3)^{*}} A_{3} P(b / W)^{3} \sqrt{\pi b}=0.35152(-1.7545 P)\left(0.4^{3}\right) \sqrt{0.4 \pi}=-0.0443 P
\end{aligned}
$$

In this example, there is also pressure acting on the crack face. Therefore an additional term for the uniform pressure is required.

$$
\begin{aligned}
& K_{I}=K_{I}^{(0)^{*}} P \sqrt{\pi b}+\sum_{i=0}^{3} K_{I}^{(i)} \\
& K_{I}=0.7032 P \sqrt{0.4 \pi}+(1.0801-0.6711+0.2726-0.0443) P \\
& K_{I}=1.4256 P \\
& K_{I} /(P \sqrt{\pi b})=1.2717
\end{aligned}
$$

Figure 2.21 shows the normalized stress intensity factors obtained using the influence function method together with those obtained directly as presented in Section 2.3.2. It can be seen from the plot that the results are in very good agreement, with deviations of less than $1 \%$. This small amount of error stems from the estimation of the third degree polynomial for the uncracked stress distribution.

\subsection{Conclusions}

The BEM formulations for three-dimensions and axisymmetry in elastostatics, along with their numerical applications, were reviewed. By solving a number of test problems, it was demonstrated that the BEM is an efficient tool for solving stress concentration and linear elastic fracture mechanics problems. With this validation, a spherical vessel with a semielliptical surface crack subjected to internal pressure will be analyzed next. 


\begin{tabular}{|c|c|c|c|c|}
\hline & \multicolumn{3}{|c|}{$\sigma_{y} / \sigma$} \\
\hline$R_{a} / R_{b}$ & AXBIE2S & BIE3D6 & FEM & Wang (2002) \\
\hline 0.2 & 2.0693 & 2.0490 & 2.063 & 2.053 \\
0.4 & 2.2173 & 2.2052 & 2.205 & 2.183 \\
0.6 & 2.7198 & 2.7092 & 2.686 & 2.6 \\
\hline
\end{tabular}

Table 2.1. Stress concentration factors, $\sigma_{y} / \sigma$, for a cylindrical bar with a spherical cavity: $H / R_{b}=2$

\begin{tabular}{|c|c|c|c|c|c|c|c|c|c|}
\hline \multirow{3}{*}{$K_{I}^{(\mathrm{i})^{*}}$} & \multicolumn{8}{|c|}{$\phi$} \\
\cline { 2 - 9 } & $0^{\circ}$ & $12.64^{\circ}$ & $25^{\circ}$ & $35.20^{\circ}$ & $45^{\circ}$ & $56.73^{\circ}$ & $68^{\circ}$ & $80.30^{\circ}$ & $90^{\circ}$ \\
\hline$K_{I}^{(0)^{*}}$ & 0.70321 & 0.70214 & 0.69040 & 0.68182 & 0.67188 & 0.66024 & 0.63356 & 0.66976 & 0.65948 \\
$K_{I}^{(1)^{*}}$ & 0.49234 & 0.48424 & 0.45697 & 0.42364 & 0.38143 & 0.31553 & 0.23702 & 0.16381 & 0.10107 \\
$K_{I}^{(2)^{*}}$ & 0.40378 & 0.39119 & 0.35369 & 0.30727 & 0.25223 & 0.17775 & 0.10717 & 0.06071 & 0.03940 \\
$K_{I}^{(3)^{*}}$ & 0.35152 & 0.33516 & 0.29020 & 0.23638 & 0.17687 & 0.10757 & 0.05425 & 0.02931 & 0.02075 \\
\hline
\end{tabular}

Table 2.2. Normalized influence coefficients, $K_{I}^{(\mathrm{i})} / A_{i}(b / W)^{\mathrm{i}} \sqrt{ }(\pi b)$, for a thick-wall cylinder with a semi-elliptical surface crack: $k=2.5, b / a=0.8, b / W=0.4$ 


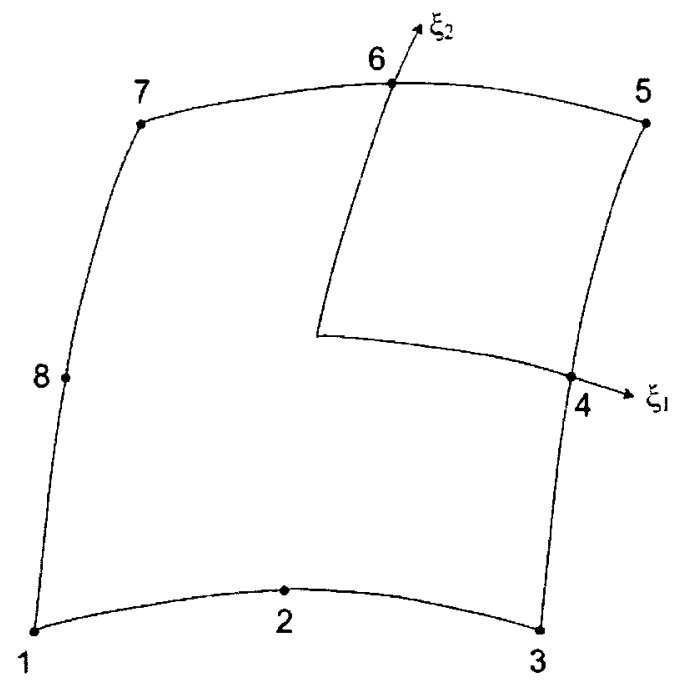

Figure 2.1. Quadratic isoparametric eight-node quadrilateral element for threedimensional BEM analysis

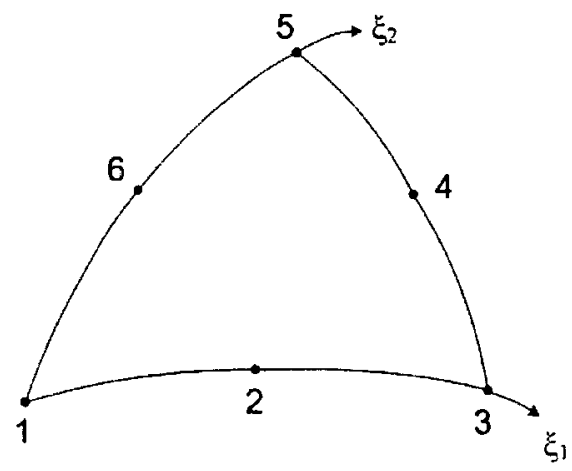

Figure 2.2. Quadratic isoparametric six-node triangular element for three-dimensional BEM analysis

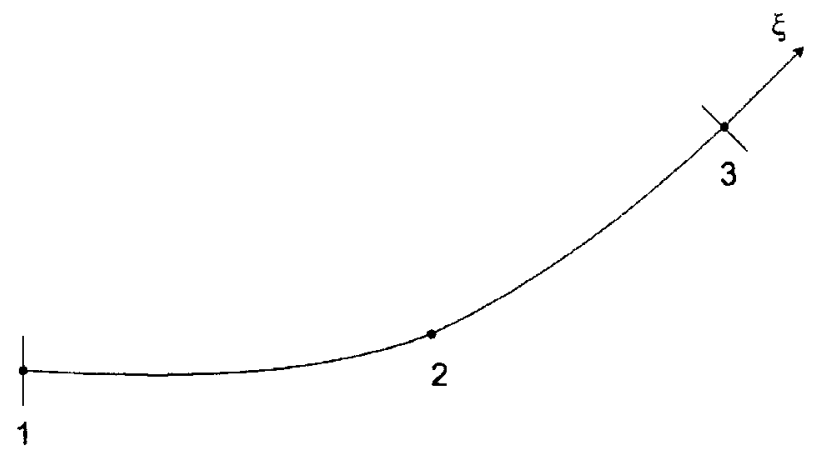

Figure 2.3. Quadratic isoparametric three-node line element for axisymmetric BEM analysis 


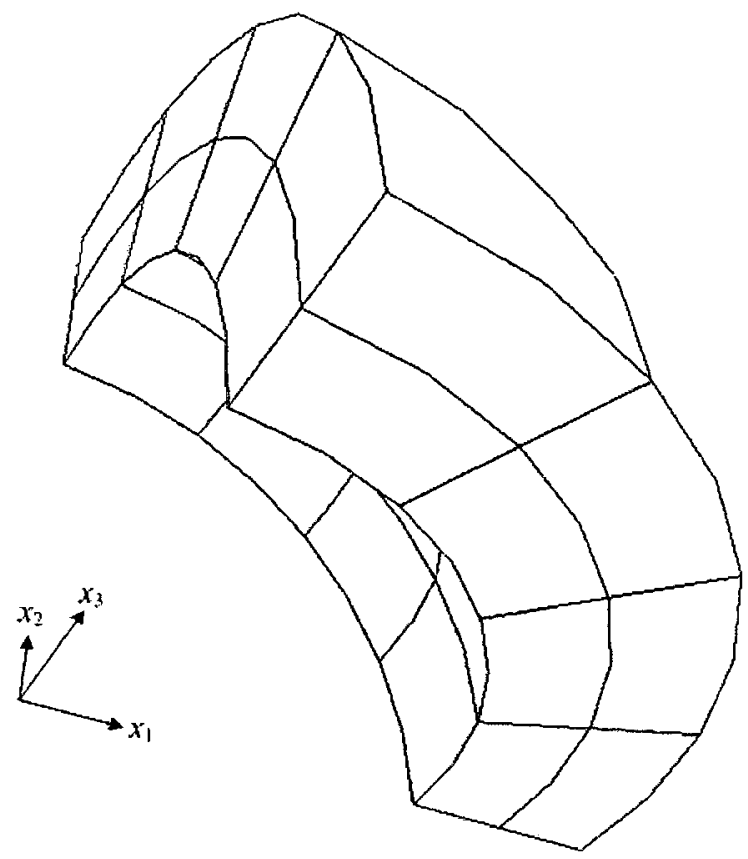

(a)

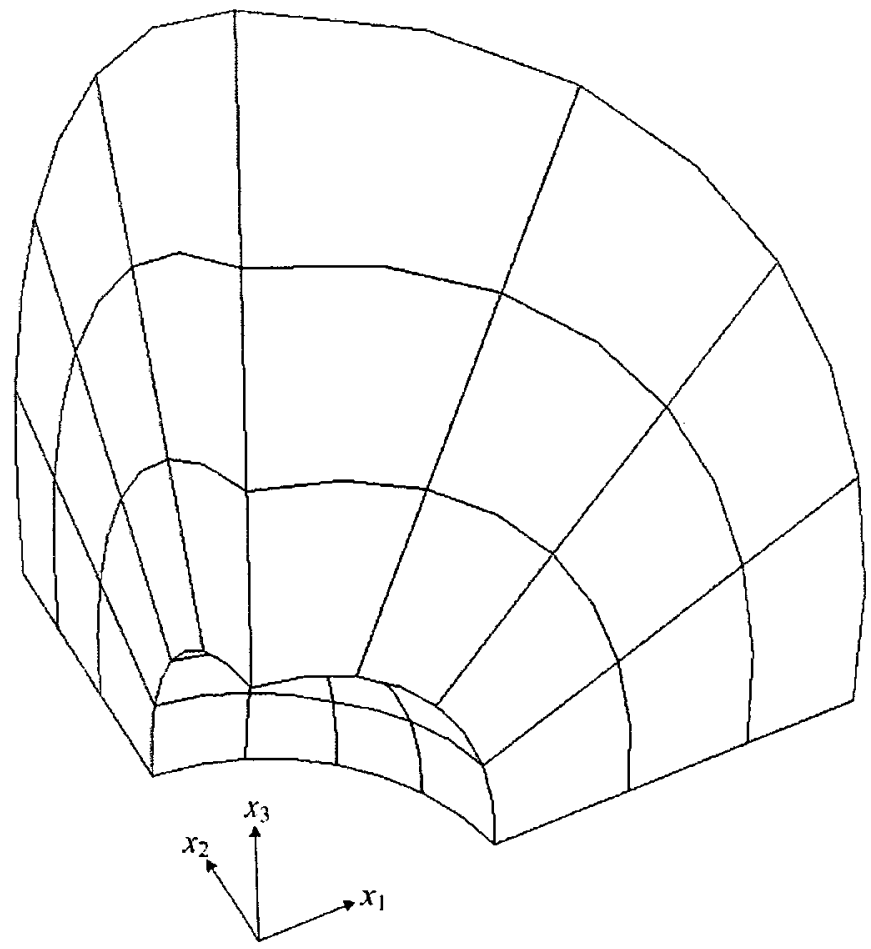

(b)

Figure 2.4. Three-dimensional BEM mesh for a spherical vessel: (a) $k=1.5 ;$ (b) $k=3$ 


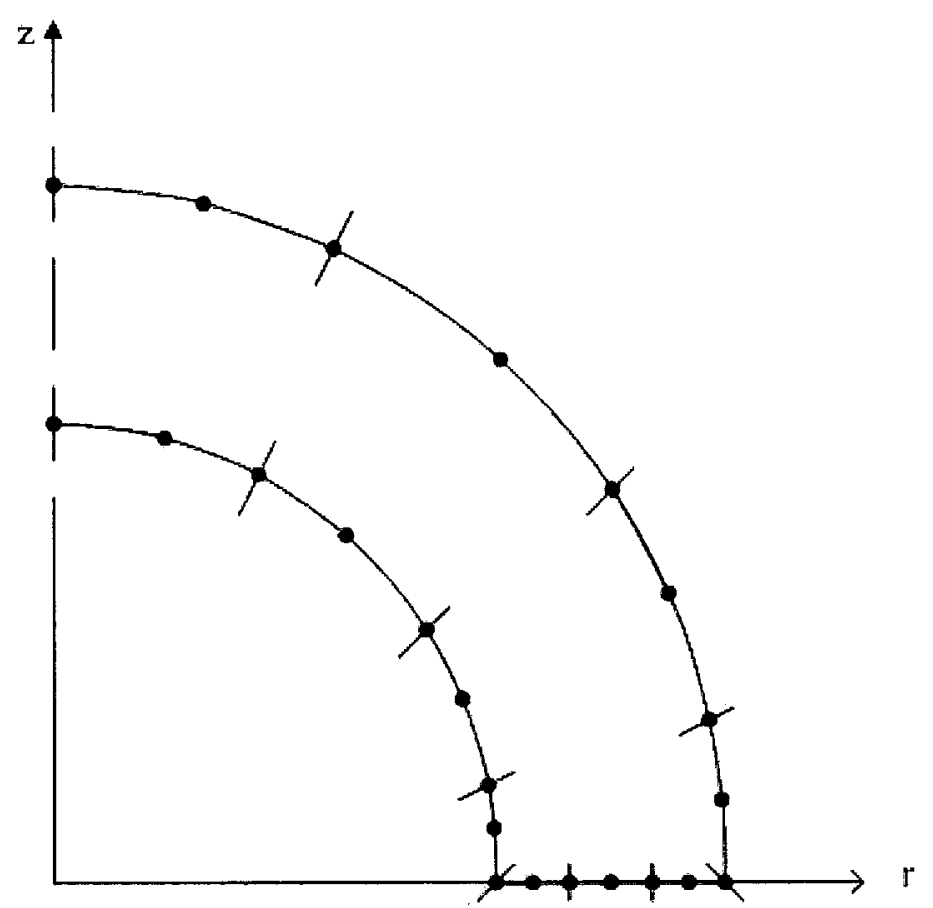

(a)

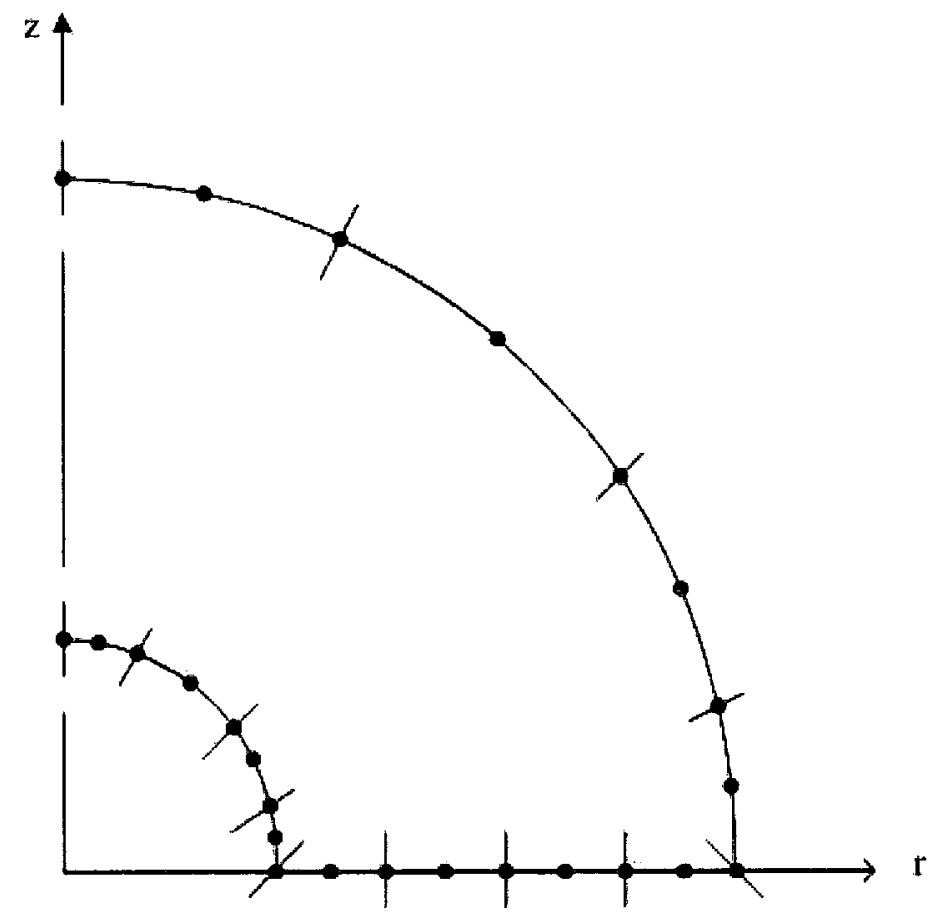

(b)

Figure 2.5. Axisymmetric BEM mesh for a spherical vessel: (a) $k=1.5$; (b) $k=3.0$ 


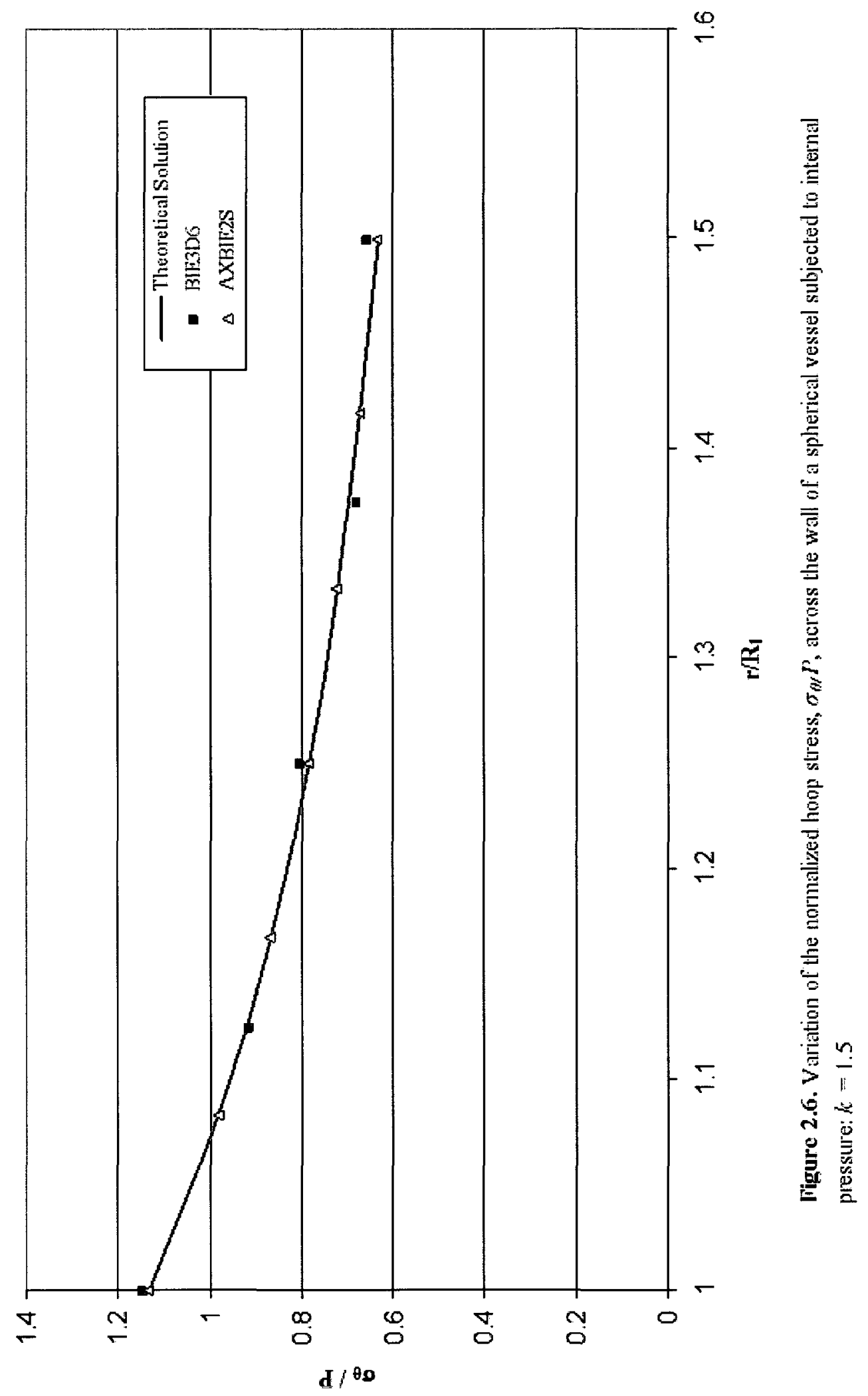




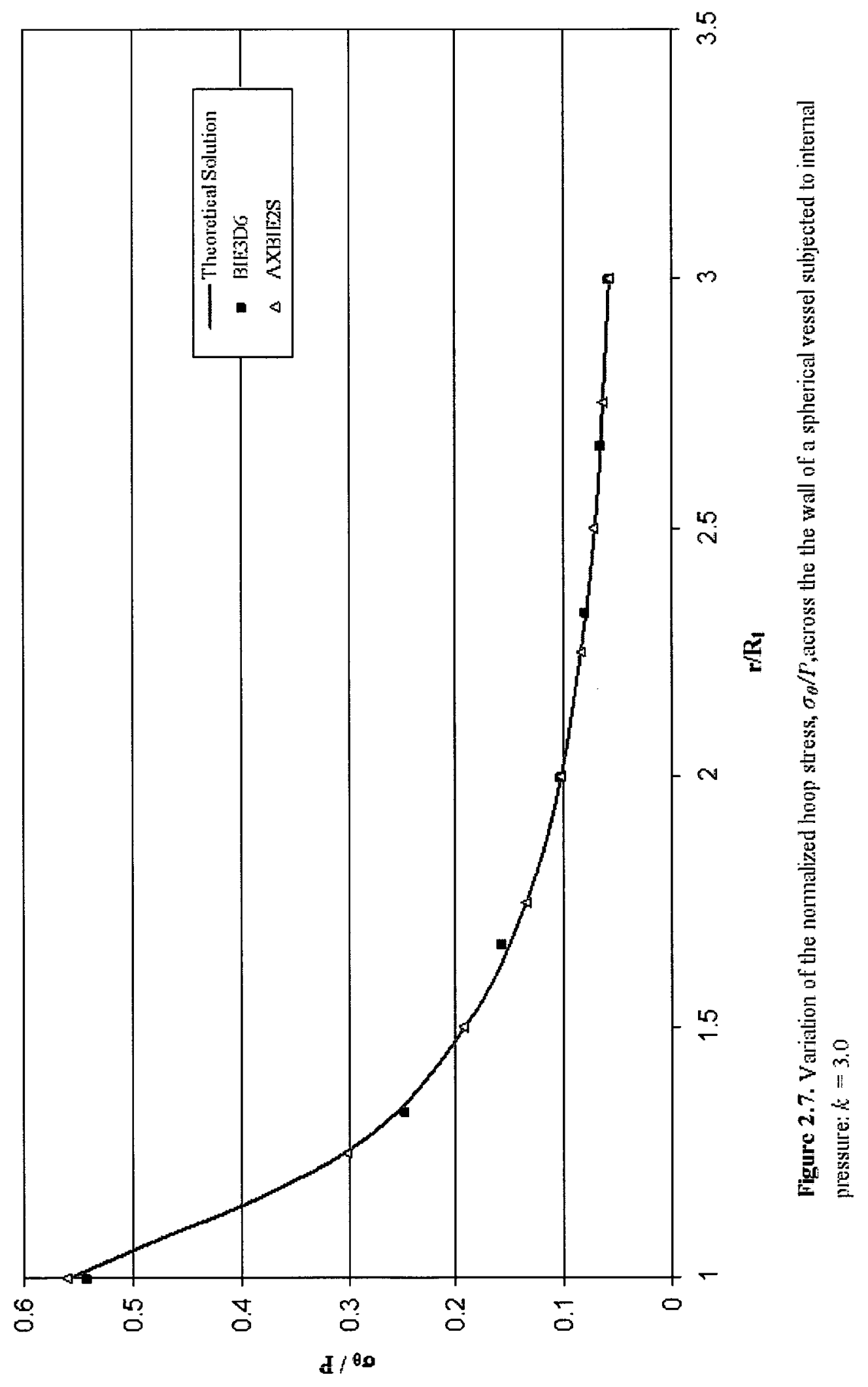




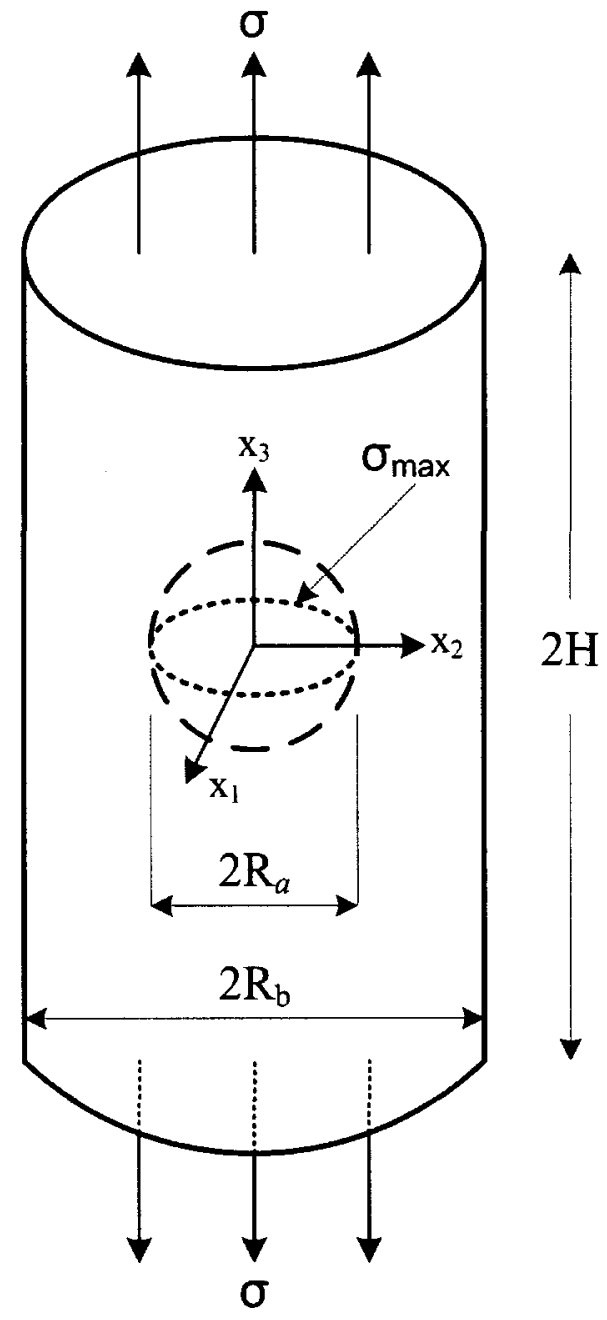

Figure 2.8. Cylindrical bar with a spherical cavity 


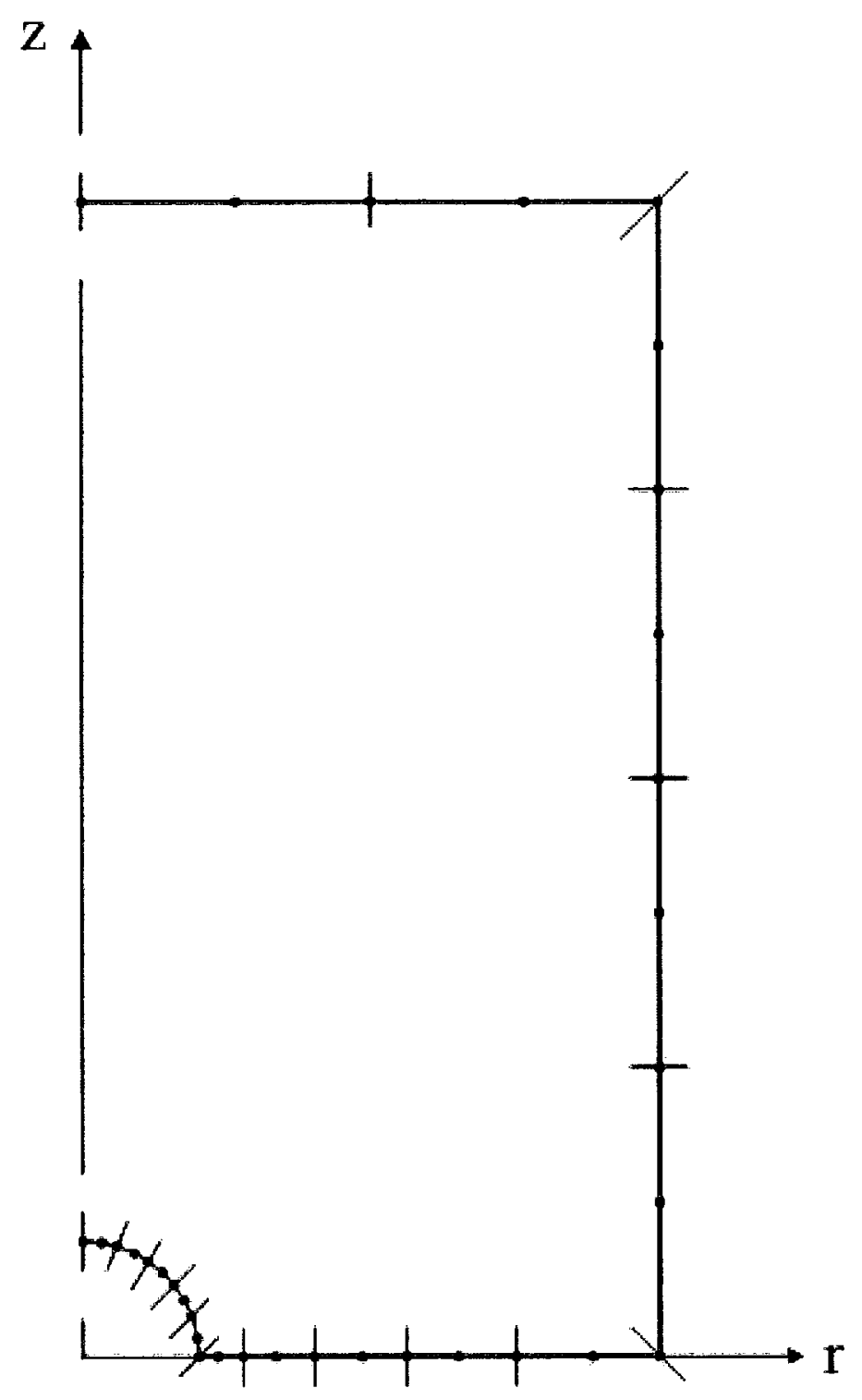

Figure 2.9(a). Axisymmetric BEM mesh for a cylindrical bar with a spherical cavity: $H / R_{b}=2, R_{a} / R_{b}=0.2$ 


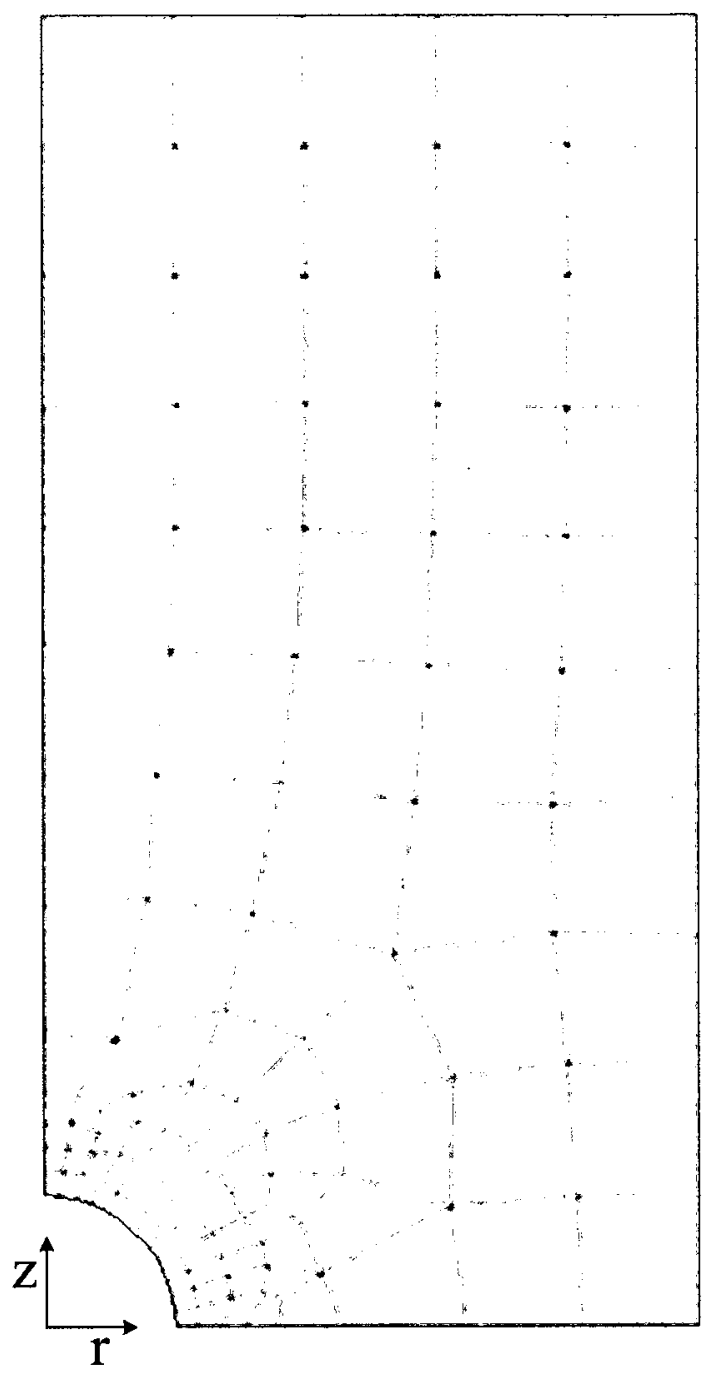

Figure 2.9(b). Axisymmetric FEM mesh for a cylindrical bar with a spherical cavity: $H / R_{b}=2, R_{a} / R_{b}=0.2$ 


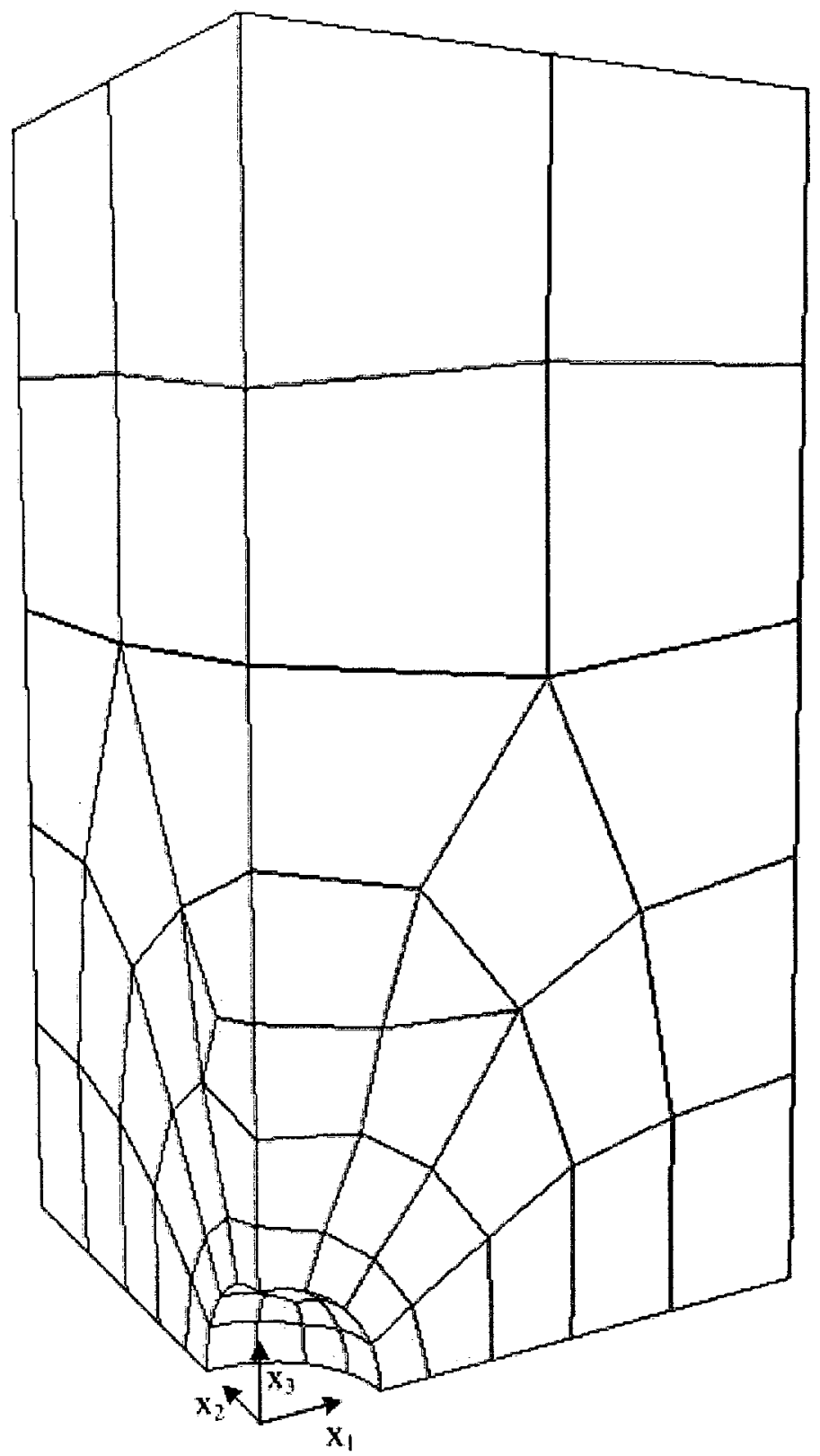

Figure 2.10. Three-dimensional BEM mesh for a cylindrical bar with a spherical cavity: $H / R_{b}=2, R_{a} / R_{b}=0.2$ 

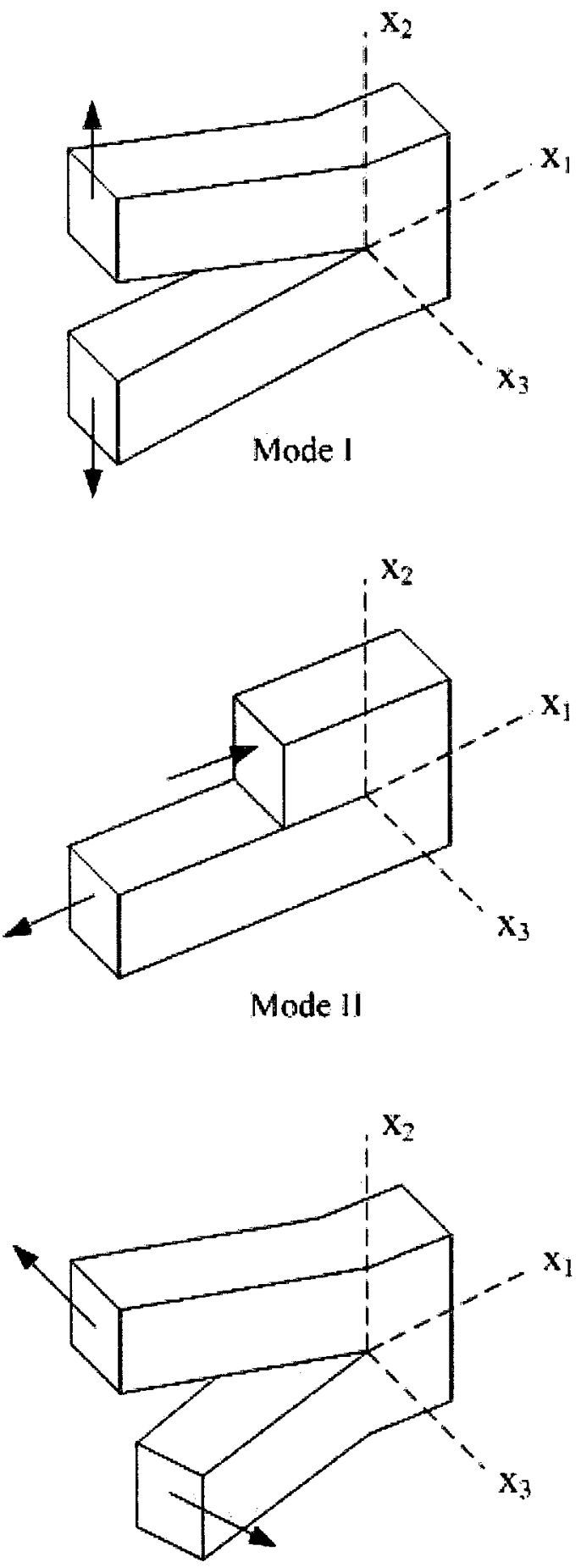

Mode III

Figure 2.11. Crack deformation modes 


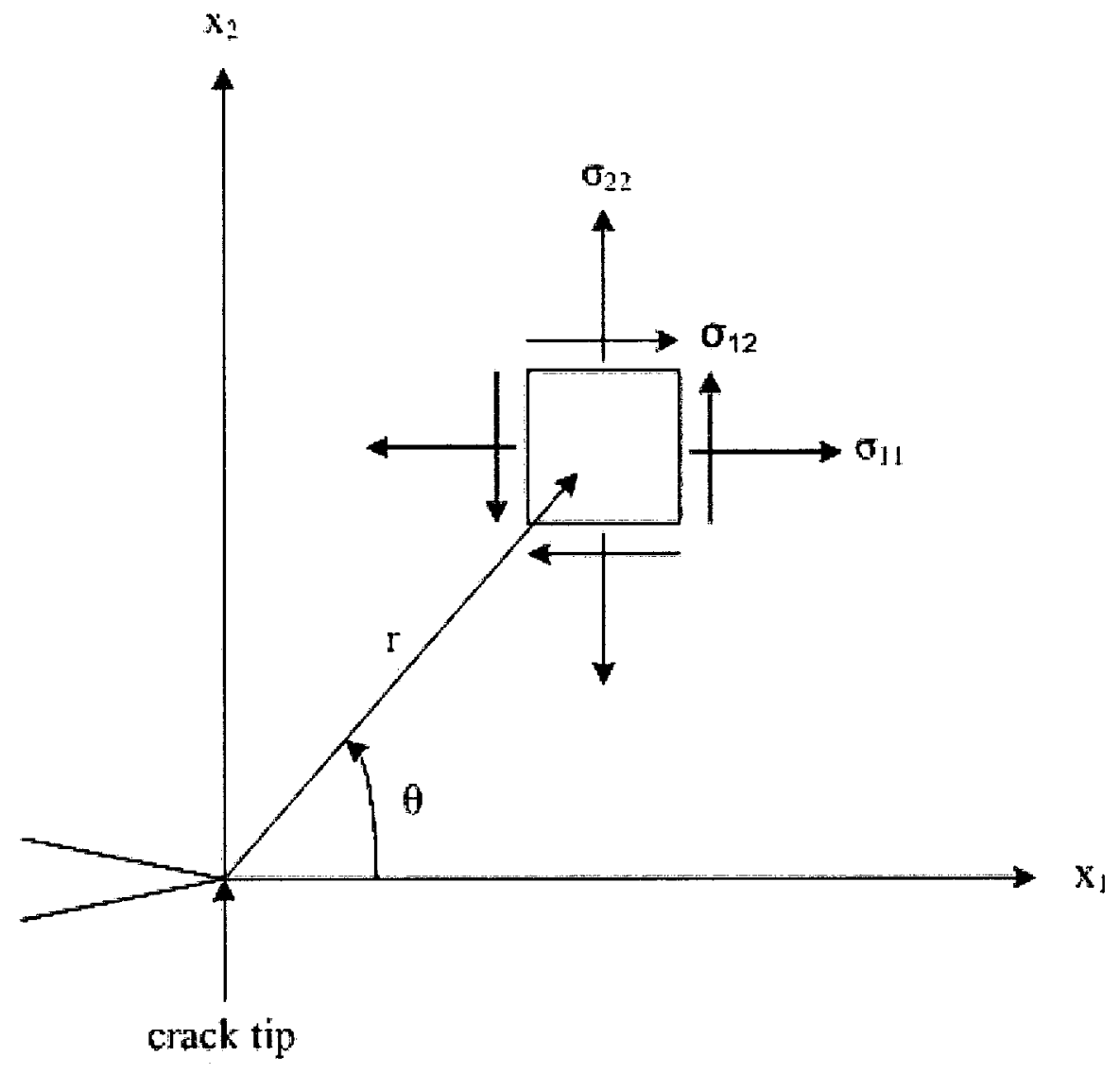

Figure 2.12. Stresses near the crack tip 


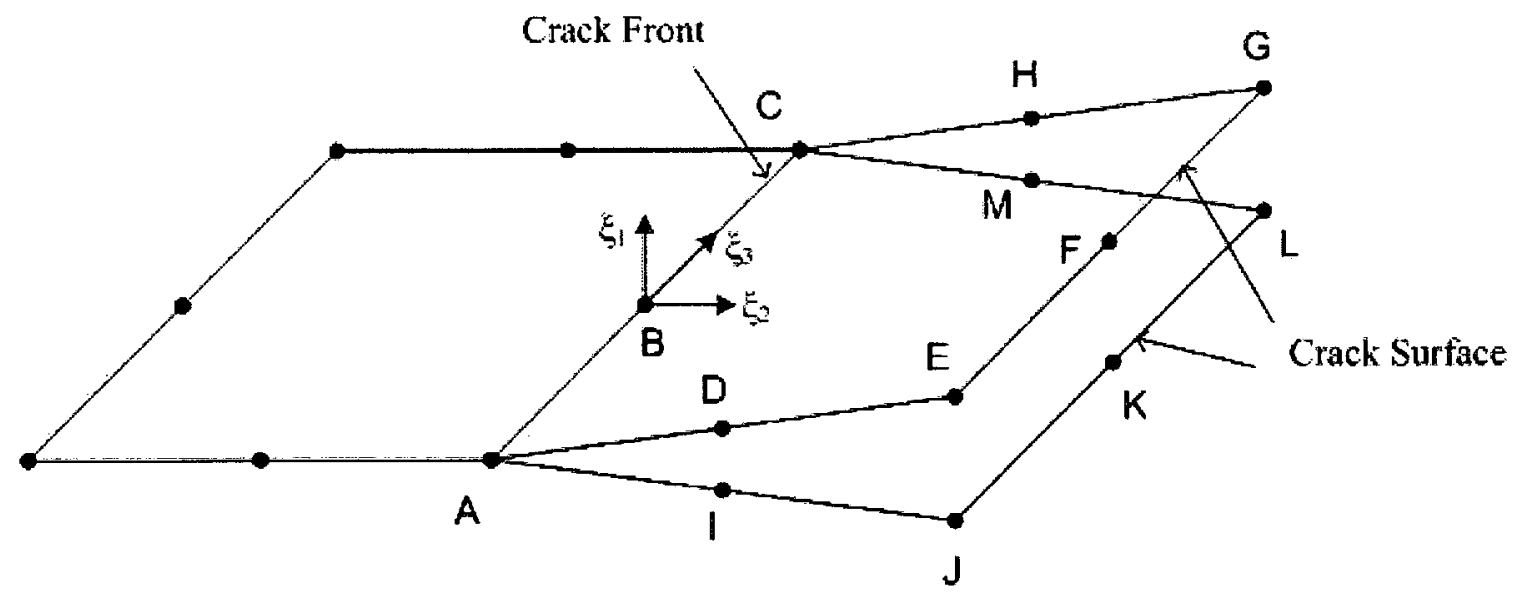

Figure 2.13. Crack element notation for determining stress intensity factors directly 


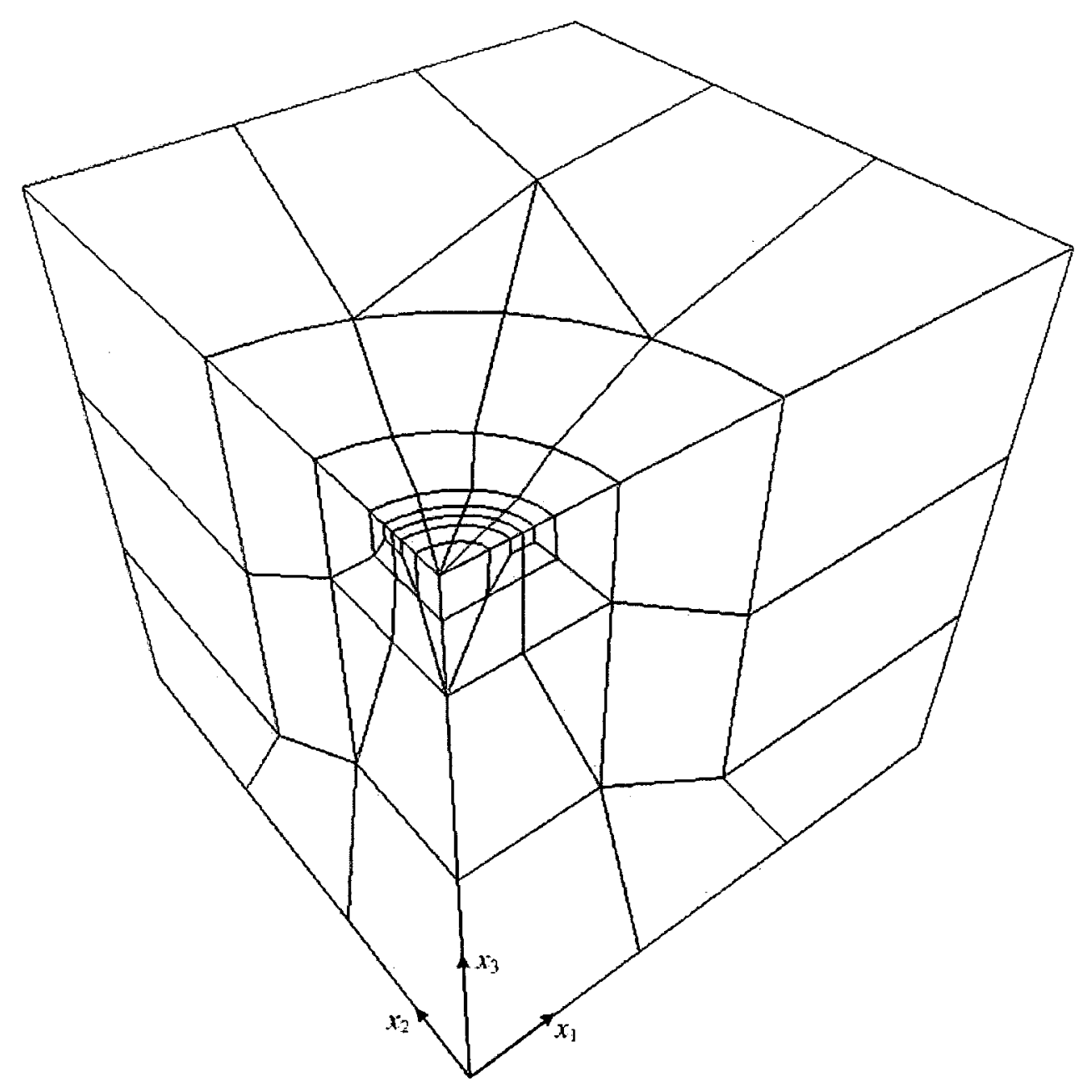

Figure 2.14. Three-dimensional BEM mesh for an elliptical crack in an infinite body: $b / a=0.8$ 


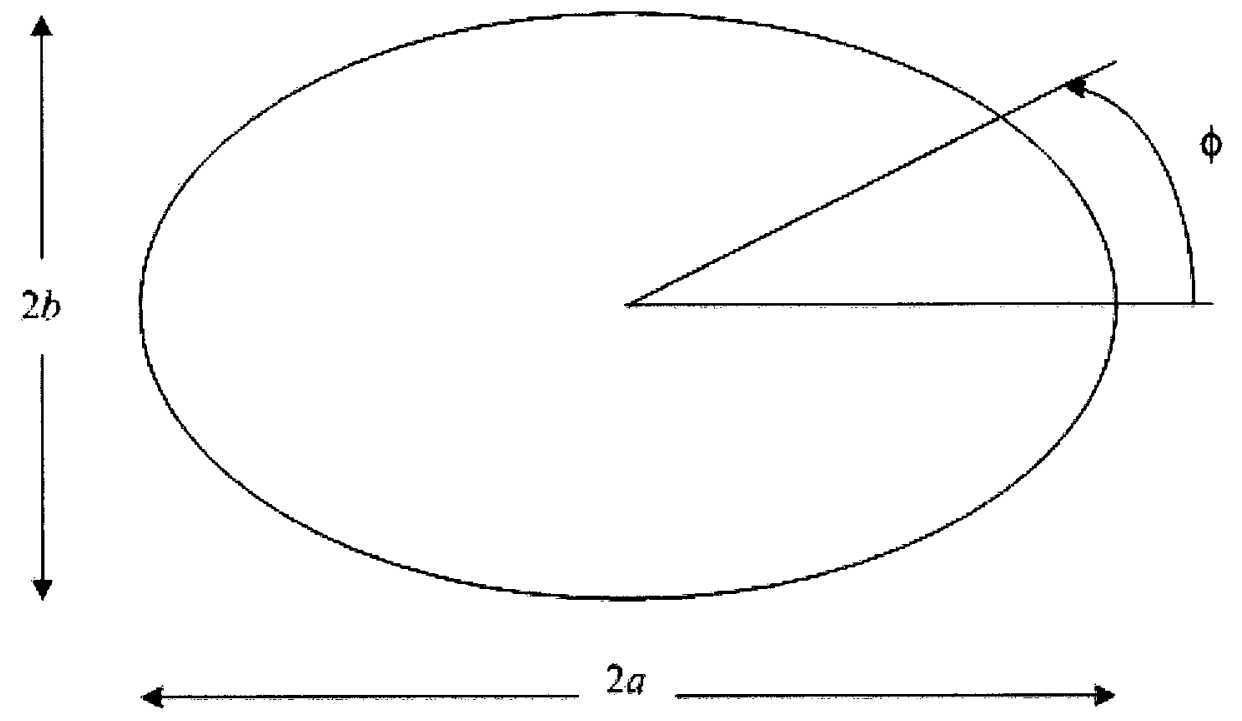

Figure 2.15. Definition of the parameters for an elliptical crack in an infinite body 


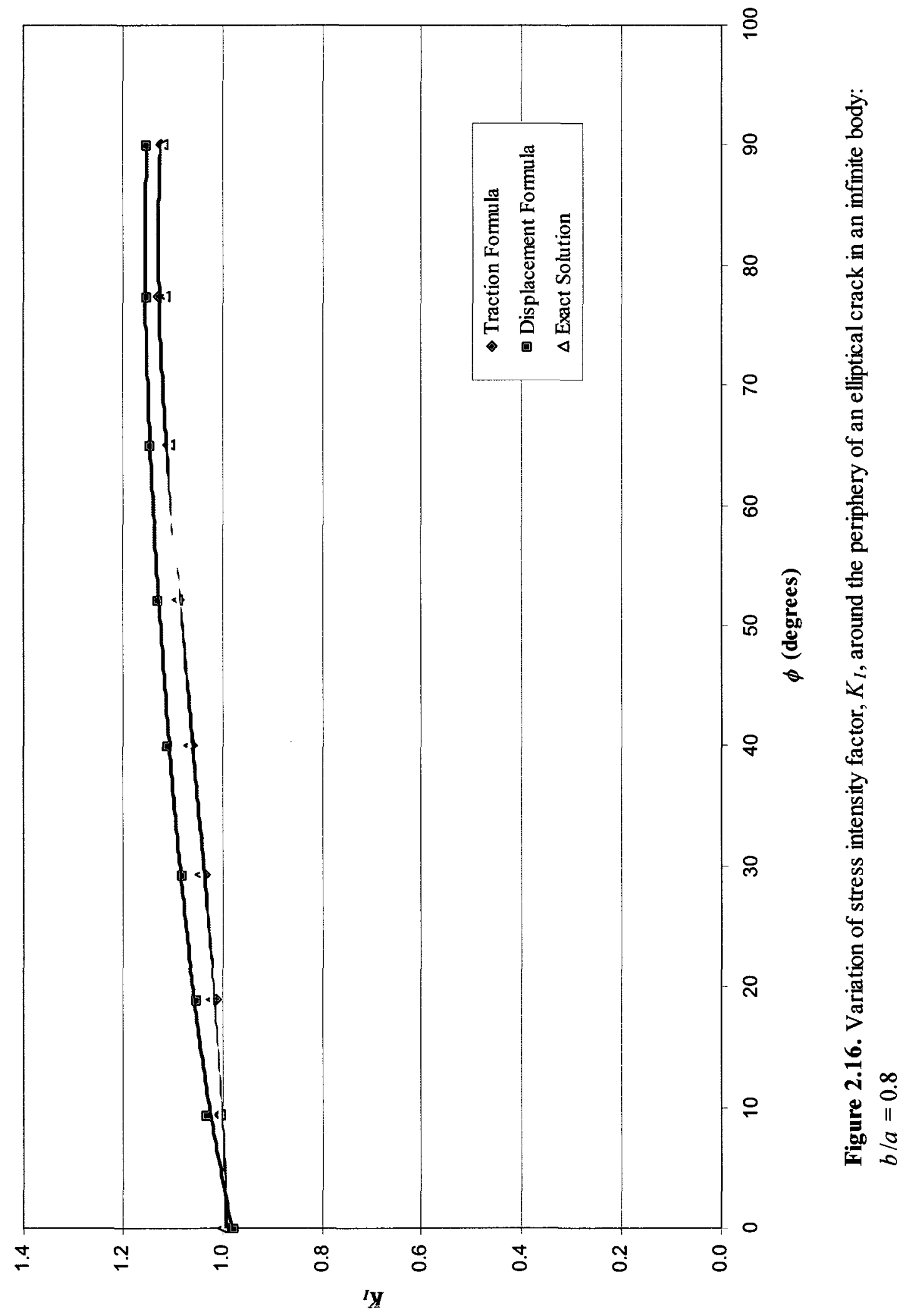




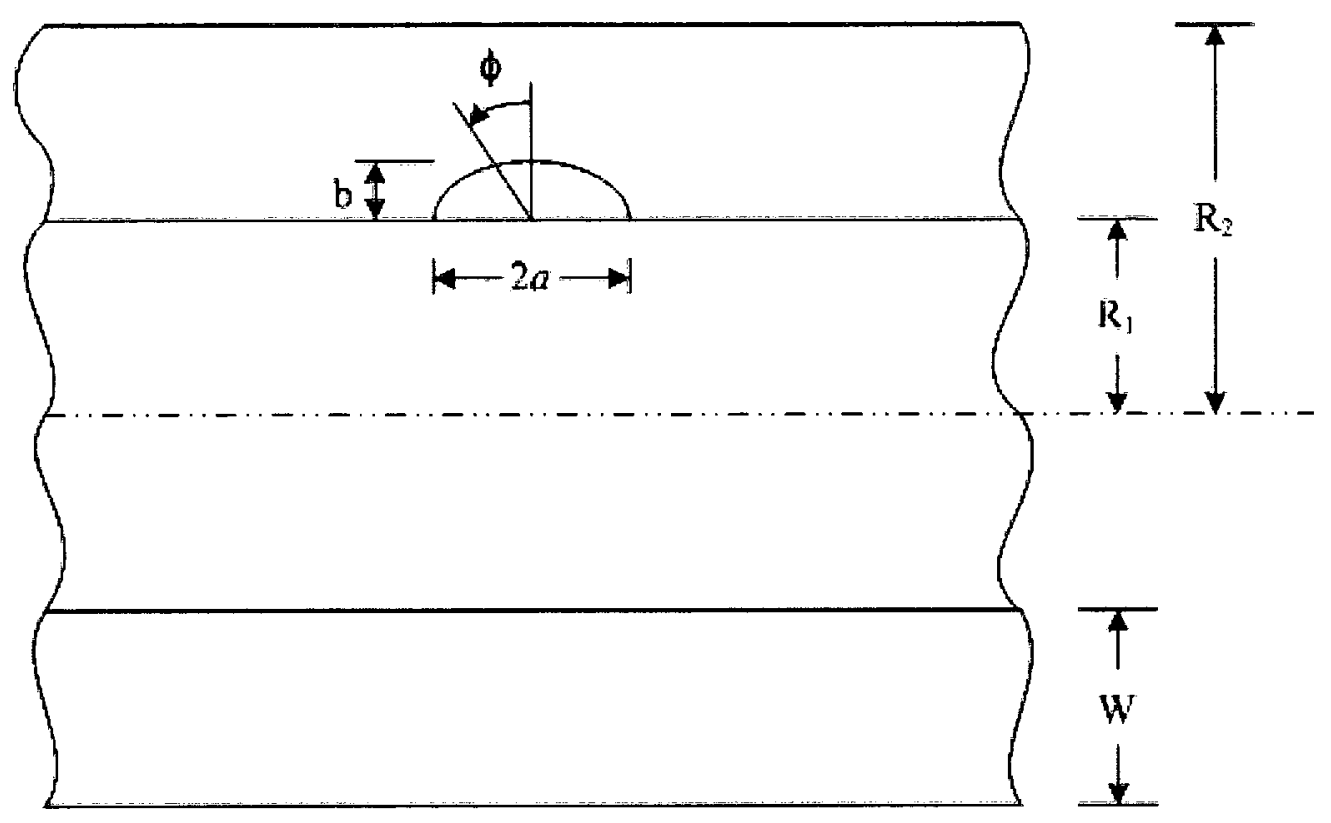

Figure 2.17. Diametrical section of a thick-wall cylinder with a semi-elliptical crack 


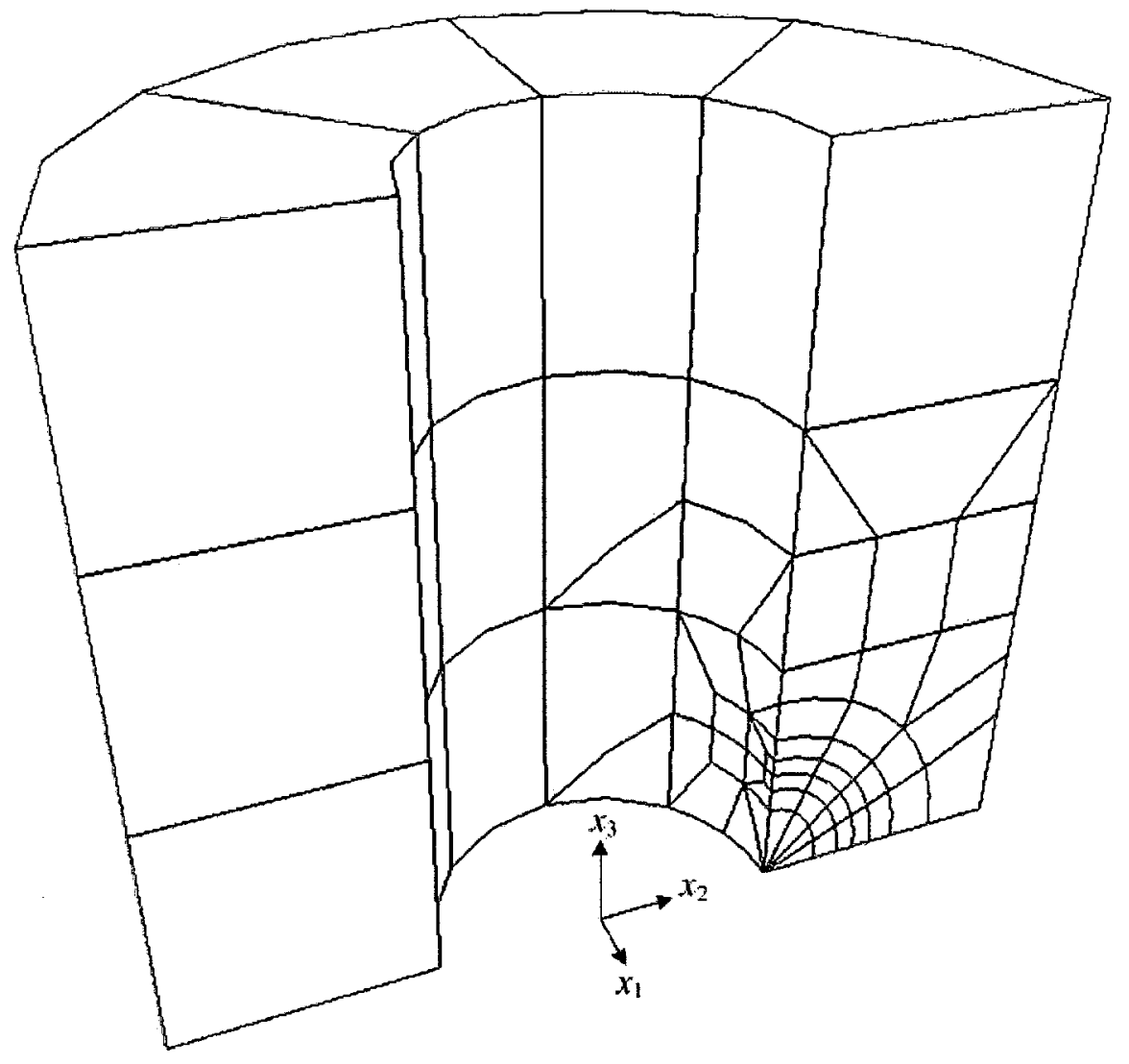

Figure 2.18. Three-dimensional BEM mesh for a thick-wall cylinder with a semielliptical crack: $k=2.5, b / a=0.8, b / W=0.4$ 


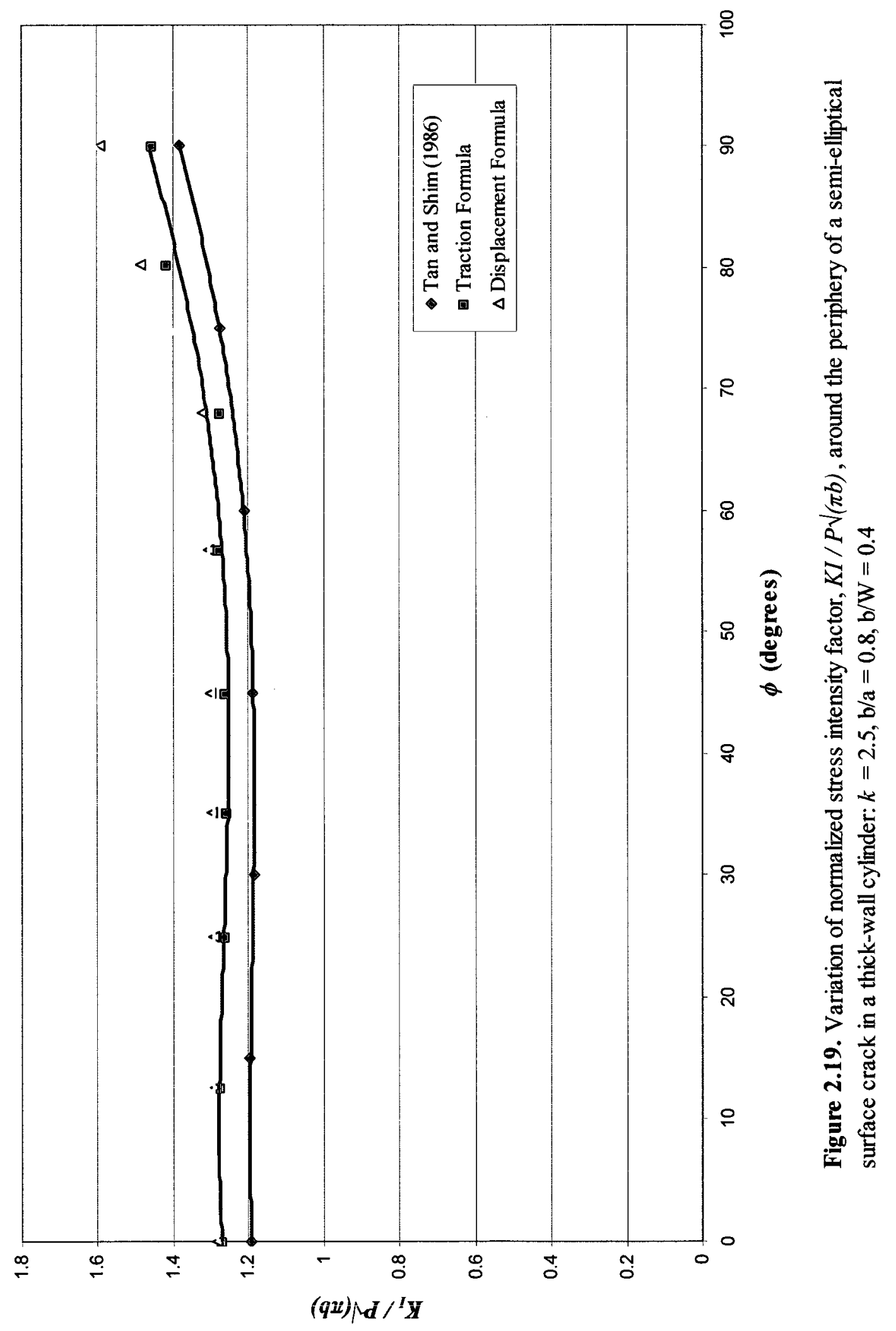




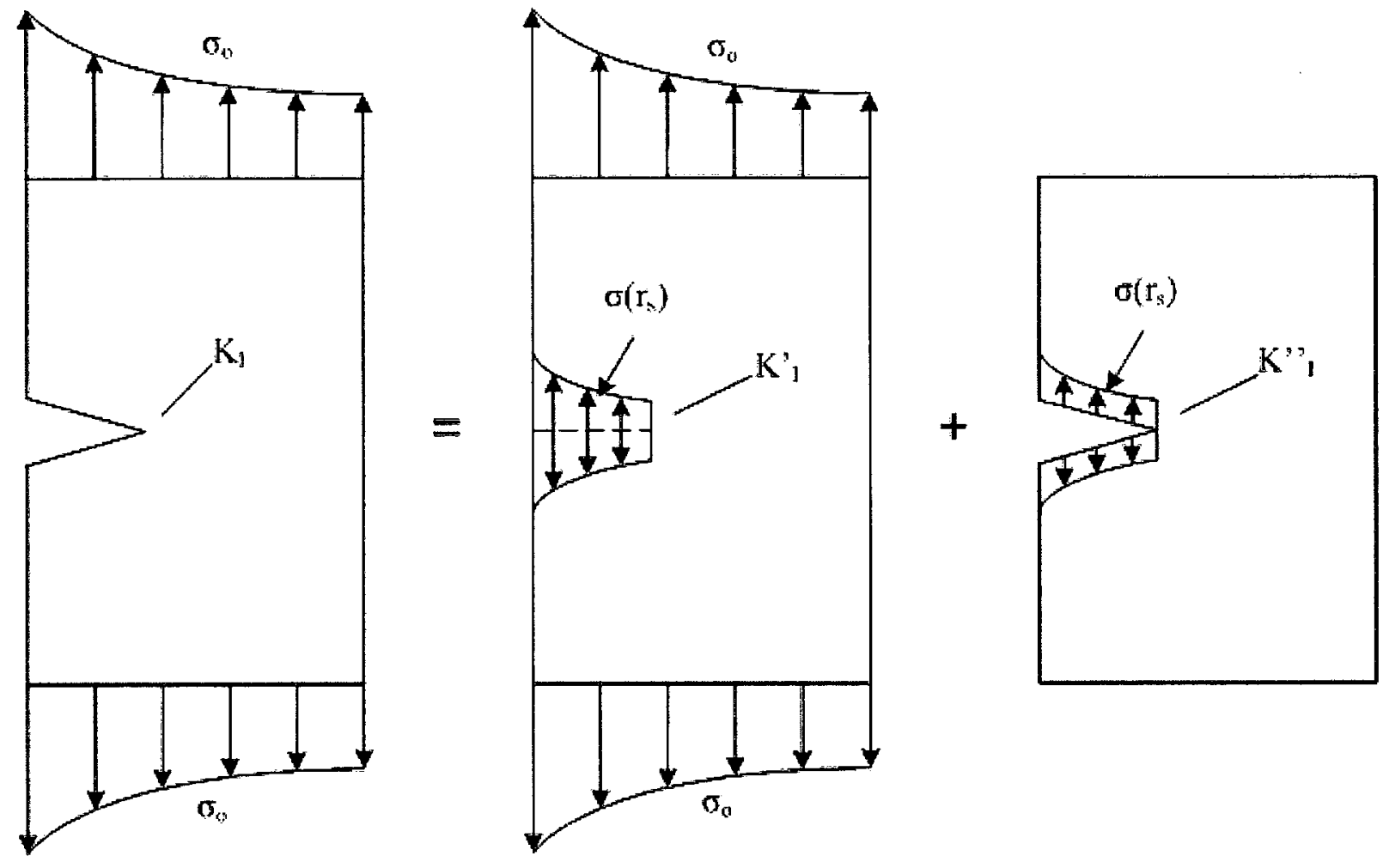

Figure 2.20. Superposition for determining stress intensity factors 


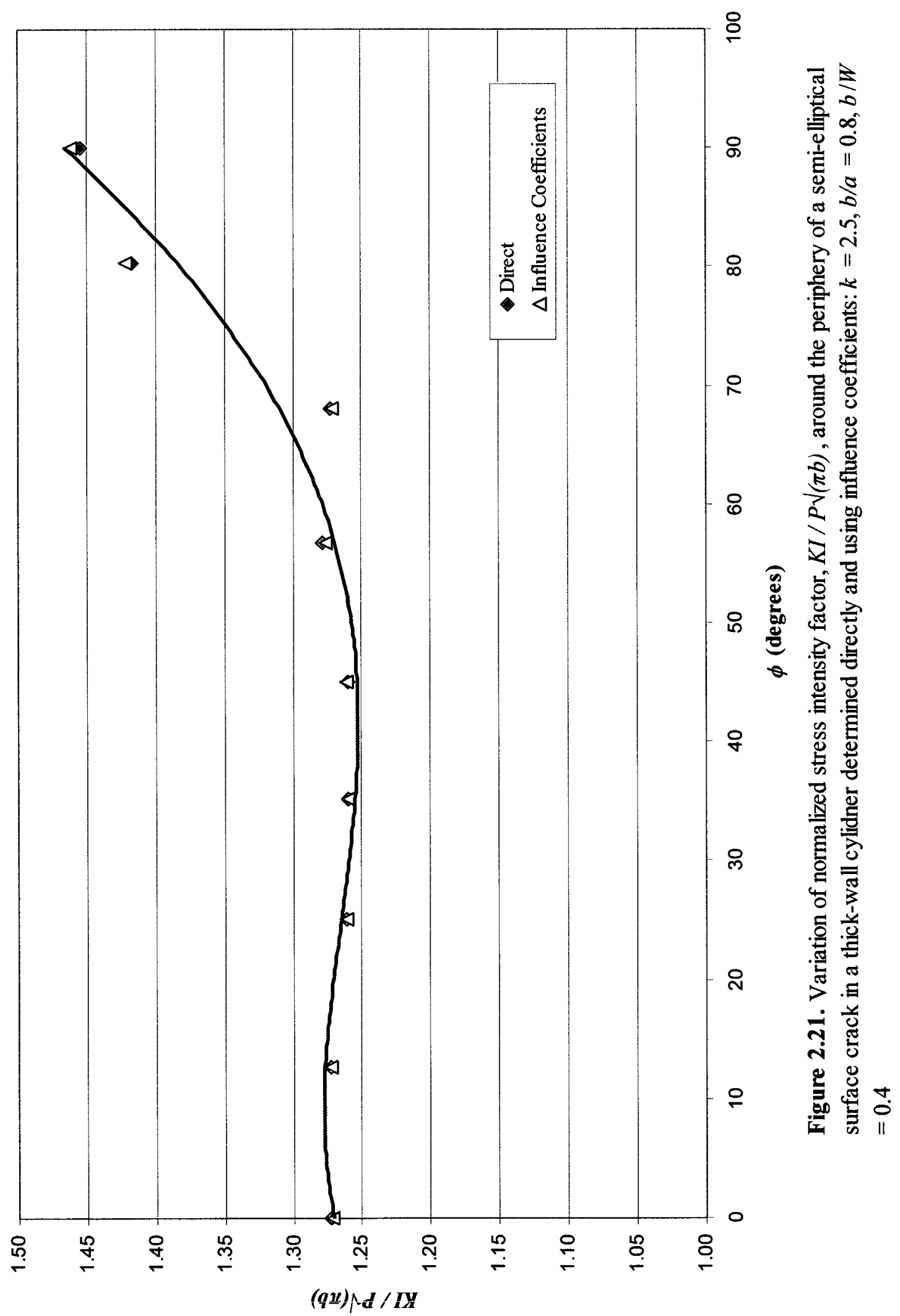

40 


\section{Chapter 3}

\section{Spherical Vessel with an Internal Surface Crack}

\subsection{Introduction}

In this chapter, a spherical vessel with an internal surface crack, subjected to an internal pressure is investigated. The physical problem is first defined, and the three-dimensional BEM modelling process is described. Stress intensity factors are determined directly for a wide range of geometric cases. Influence coefficients are also obtained to calculate this fracture parameter.

\subsection{Problem Definition}

Figure 3.1 shows the problem of a spherical vessel with an internal surface crack. The spheres investigated in this study have radius ratios of

$$
k=R_{2} / R_{l}=1.5,2,2.5,3
$$

containing internal surface cracks with aspect ratios of

$$
b / a=1.0,0.8,0.6
$$

and relative depths of

$$
b / W=0.2,0.4,0.6,0.8
$$

The only combinations not considered are $k=2.5, b / a=0.6, b / W=0.8$, and $k=3, b / a=$ $0.6, b / W=0.8$, where the crack would almost extend through the entire thickness of the sphere; it is unlikely that this size of crack would be allowed to be reached in practice. 
Stress intensity factors were determined for 46 cases of the problem of a cracked thickwalled sphere under internal pressure, $P$. It is assumed that in all the cases treated, the internal pressure also acts on the crack faces, which represents the worst load case for this crack geometry. Poisson's ratio was taken to be 0.3 .

\subsubsection{Numerical Model}

Similar three-dimensional modelling strategies from the numerical examples in Section 2.3 were used to model the spherical vessel with an internal surface crack. Taking advantage of symmetry, only one-quarter of the problem was modelled, as shown for $k=$ $1.5, b / a=0.8, b / W=0.4$ in Figure 3.2. The size of the numerical model varied from 96 elements and 278 nodes to 111 elements and 319 nodes, depending on the geometry. The length, $l$, of the crack elements normal to the crack front was set to $0.15 a$ for all cases (see, e.g. Luchi and Rizzuti (1987)). The elements were then gradually increased in size to match the larger elements in the model away from the crack. The number of elements along the crack front varied from four to six, depending on the accuracy of the obtained results. Assessment of the results is discussed in the next section. It was found that for relatively larger crack sizes, it was necessary to add more elements along the crack front near the free surface. Displacement was constrained on the planes of symmetry in the normal directions, which prevented rigid body motion in the $\mathrm{x}_{2}$ - and $\mathrm{x}_{3}$ - directions. To restrict rigid body motion in the $\mathrm{x}_{1}$ - direction, displacement constraints in this direction were applied to the nodes at the intersection of the exterior surface and the $\mathrm{x}_{2}-\mathrm{x}_{3}$ plane. Here the tractions in the $\mathrm{x}_{1}$ - direction are zero, thus these nodal constraints only restrict rigid body motion. A uniform pressure, $P$, was applied to the internal surface and crack face.

\subsubsection{Results}

Since a theoretical solution does not exist for this problem, the accuracy of the results was assessed by comparing the stress intensity factors determined using the traction formula and displacement formulas. If good agreement was established between the 
obtained values, the mesh employed was assumed to be acceptable for the problem. The normalized stress intensity factors, $K_{I} / P \sqrt{ }(\pi b)$, determined using both the traction formula and displacement formulas are presented with respect to the elliptical angle, $\phi$, in Tables 3.1 to 3.12 . It is worth noting here that since the internal surface of the spherical vessel is curved in the diametrical plane, its intersection with the crack occurs at a different elliptical angle for each case, unlike the straight cylinder from Section 2.3.2. Hence the different range of $\phi$ for each geometric combination. It is clear that around the periphery of the crack front, with the exception of the free surface and the adjacent mid-side node, there is very good agreement between the results obtained using the traction and displacement formulas, with discrepancies between nodal values within $3 \%$. The large discrepancies between the values for those points at the free surface seen in the previous chapter were again evident here; this was attributed to the $1 / \sqrt{ } r$ traction singularity not being valid at the intersection of the crack and the free surface. These results should thus be used with caution. In some cases, the result for the adjacent mid-side node was also affected. However, refining the mesh or adding another crack element near the free surface generally resulted in a discrepancy between the results obtained using both formulas of well within $6 \%$ at this nodal location. The values obtained using the displacement formula are presented graphically throughout the present study.

The effects of crack depth, $b / W$, on the normalized stress intensity factor of a semicircular crack are presented graphically in Figures 3.3 to 3.6, where it is apparent that it increases with relative crack depth around most of the crack periphery, as denoted by $\phi$. It can be seen in Figure 3.3 for case $k=1.5, b / a=1$, that the normalized stress intensity factor remains somewhat constant around the semi-circular crack until approximately $\phi=$ $45^{\circ}$, where as expected, it increases as the free surface is approached. Interestingly, at the free surface, the normalized stress intensity factor for the larger crack depths, $b / W=0.6$ and 0.8 , begins to level off. This trend becomes more evident for all crack depths as the radius ratio, $k$, of the vessel increases. Figures 3.4 to 3.6 show the normalized stress intensity factor for the thicker spherical vessels with a semi-circular crack. For $k=2$, the normalized stress intensity factors are generally uniform, except for a minor increase at approximately $\phi=60^{\circ}$, and a gradual decrease at the free surface. As shown, this decrease 
in stress intensity factor near the free surface becomes more abrupt for the $k=2.5$ and 3 vessels.

Figures 3.7 to 3.14 present the normalized stress intensity factors if the surface crack is of the semi-elliptical form. As with the semi-circular cases, the $b / W=0.2$ and 0.8 crack depths generally resulted in larger values around most of the crack periphery. Although it is evident that lowering the aspect ratio generally increases the normalized stress intensity factors. The $k=1.5, b / a=0.8$ plot, Figure 3.7, shows that the normalized stress intensity factor slowly decreases until approximately $\phi=70^{\circ}$, where it increases to the free surface. If the aspect ratio of the crack is decreased further to $b / a=0.6$, similar observations can be made. Figure 3.8 shows that the normalized stress intensity factor decreases until approximately $\phi=75^{\circ}$, where it increases very rapidly to the free surface. However, these fluctuations are reduced as the radius ratio of the vessel is increased. Figures 3.9 and 3.10 show that there is less variation of the normalized stress intensity factor around the crack periphery for a vessel with a radius ratio of $k=2$. The increase in the stress intensity factor near the free surface is still present, but is more gradual than for the $k=1.5$ cases. These trends are even more evident as the radius ratio of the vessel is further increased, as shown in Figures 3.11 to 3.14. Similar trends were observed for the same crack geometry in straight tubing by Tan and Fenner (1980), and in curved tubing by Tan and Plante (2008). This is illustrated in Figure 3.15, which shows the normalized stress intensity factor variation around the periphery of a semi-elliptical surface crack $(b / a=0.8, b / W=$ 0.4 ) in a spherical vessel, straight tubing, and a $90^{\circ}$ section of curved tubing with a radius of curvature equal to five times its internal radius. Of interest to note as well is that the normalized stress intensity factor at a given position on the crack front in the sphere is lower than that of a straight tubing, which in turn is lower than that of a curved tubing. This is as expected because of the higher hoop stress acting on the crack in the straight and curved cylinders, respectively. Unexpectedly, in the spherical vessel with a radius ratio of $k=3$ with an elliptical crack spanning most of its thickness, the normalized stress intensity factor was found to decrease near the free surface, similar to the behaviours observed for semi-circular cracks. This trend was not quite expected since a straight or curved thick-walled cylinder with an internal surface crack shows no decrease in the normalized stress intensity factor near the free surface. 


\subsection{Influence Coefficients}

As explained in Section 2.4, the stress intensity factors can also be determined using the influence function method. This allows the same crack geometry under different loading conditions to be solved without performing an entirely new BEM analysis. In this section, influence coefficients will be determined for a spherical vessel with an internal surface crack. The stress intensity factors are then determined using the influence coefficients, and compared to the directly obtained values from the previous section for verification.

\subsubsection{Numerical Model}

For each geometric combination, the same mesh from the direct BEM model in the previous section was used to determine the influence coefficients. Uniform $\left(\sigma=-A_{0}\right)$, linear $\left(\sigma=-A_{1} r_{s}\right)$, quadratic $\left(\sigma=-A_{2} r_{s}{ }^{2}\right)$, and cubic $\left(\sigma=-A_{3} r_{s}{ }^{3}\right)$ traction distributions were separately applied to the crack face, where $r_{s}$ is a non-dimensional distance, as shown in Figure 3.1. Each traction distribution was normalized to reach unity at the crack depth equivalent to $b$. The normalized stress intensity factors for each distribution, $K_{I}^{(i)^{*}}$, were then determined.

\subsubsection{Results}

The complete set of influence coefficients for a spherical vessel subjected to an internal pressure with a surface crack are shown in Tables 3.14 to 3.25. These were then used to produce the stress intensity factors to assess the results. To obtain the uncracked stress distribution, $\sigma_{r s}$, the Lamé solution of a thick-walled sphere was approximated by a third degree polynomial as

$$
\sigma_{r s}=P\left(1.1297-1.0129 r_{s}+0.7715 r_{s}^{2}-0.2582 r_{s}^{3}\right)
$$


The coefficients of the polynomial were then multiplied by the appropriate normalized influence coefficients, and using equation 2.26 , they were then summed together to determine the stress intensity factors, as demonstrated below for $k=1.5, b / a=1, b / W=$ 0.4 at $\phi=0^{\circ}$.

$$
\begin{aligned}
& K_{l}^{(0)}=K_{l}^{(0) *} A_{0} P(b / W)^{0} \sqrt{\pi b}=0.650(1.1297 P) \sqrt{0.4 \pi}=0.8232 P \\
& K_{l}^{(1)}=K_{l}^{(1)^{*}} A_{1} P(b / W)^{1} \sqrt{\pi b}=0.467(-1.0129 P)(0.4) \sqrt{0.4 \pi}=-0.2121 P \\
& K_{I}^{(2)}=K_{l}^{(2)^{*}} A_{2} P(b / W)^{2} \sqrt{\pi b}=0.388(0.7715 P)\left(0.4^{2}\right) \sqrt{0.4 \pi}=0.05369 P \\
& K_{l}^{(3)}=K_{l}^{(3)^{*}} A_{3} P(b / W)^{3} \sqrt{\pi b}=0.340(-0.2582 P)\left(0.4^{3}\right) \sqrt{0.4 \pi}=-0.006298 P
\end{aligned}
$$

$$
\begin{aligned}
& K_{I}=K_{I}^{(0 * *} P \sqrt{\pi b}+\sum_{i=0}^{3} K_{I}^{(i)} \\
& K_{I}=0.650 P \sqrt{0.4 \pi}+(0.8232-0.2121+0.05369-0.006298) P \\
& K_{I}=1.3871 P \\
& K_{I} /(P \sqrt{\pi b})=1.2374
\end{aligned}
$$

The discrepancy between values obtained from the influence function method and the direct method for $k=1.5, b / a=0.8, b / W=0.6$, shown in Table 3.13, is representative of the entire set. As shown, there is excellent agreement between the two methods, with deviations within $1.5 \%$.

\subsection{Conclusions}

A number of geometric combinations of a spherical vessel with a surface crack under internal pressure were analyzed using the BEM. The effects of radius ratio, crack aspect ratio, and relative crack depth on the normalized stress intensity factors were investigated. It was determined that relative crack depths, $b / W=0.2$ and 0.8 generally produced higher values of this quantity. The normalized stress intensity factor around the periphery of a semi-circular crack tended to be relatively uniform until near the free surface, where it either gradually increased or decreased, depending on the crack depth. 
For larger relative crack depths, there would be a reduction of the normalized stress intensity factor near the free surface. However, if the surface crack was of the semielliptical form, the normalized stress intensity factor was less uniform around the crack front, with an abrupt increase near the free surface. Decreasing the aspect ratio of the crack, added to the magnitude of the variation of this quantity. As expected, increasing the radius ratio of the sphere reduced these variations and the normalized stress intensity factors. 


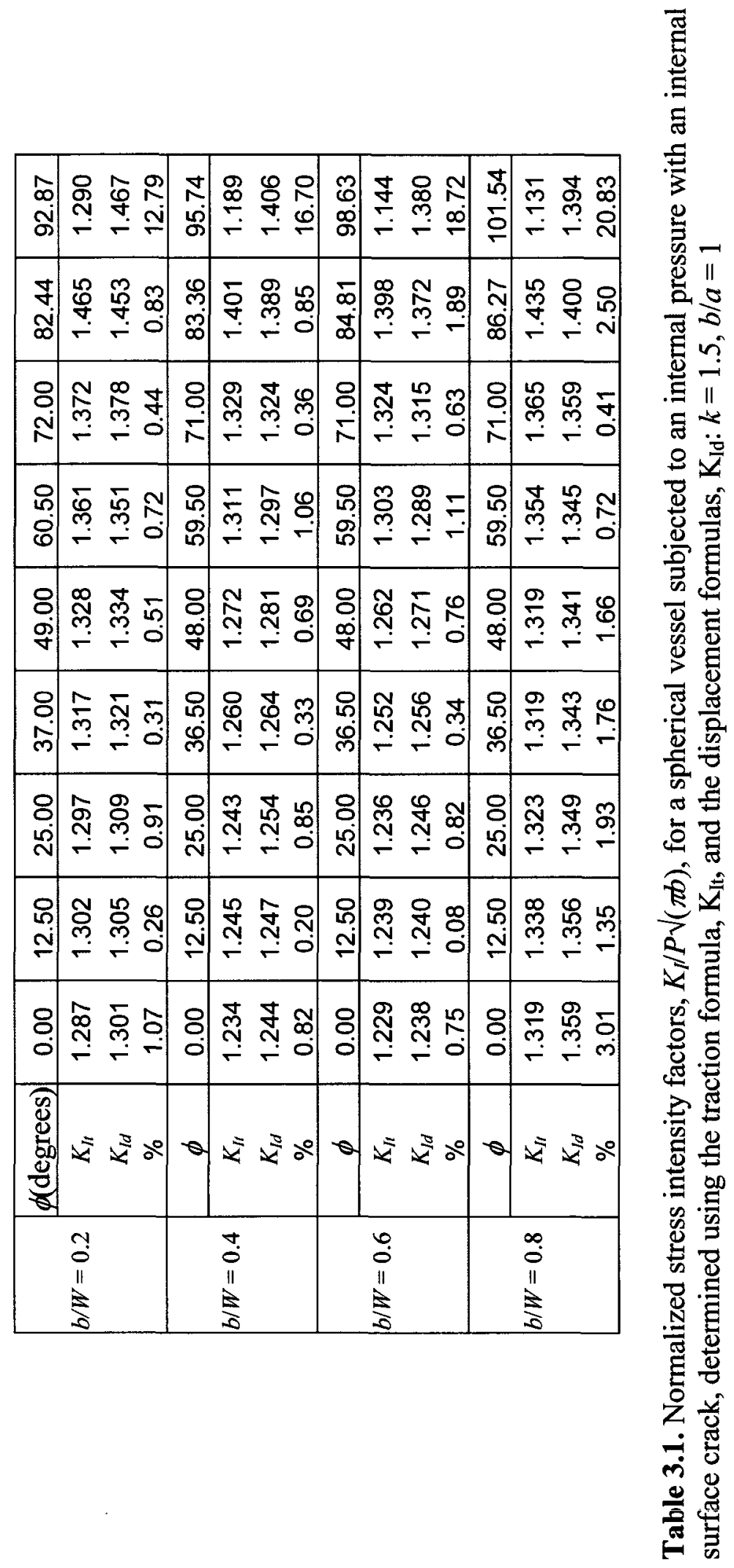




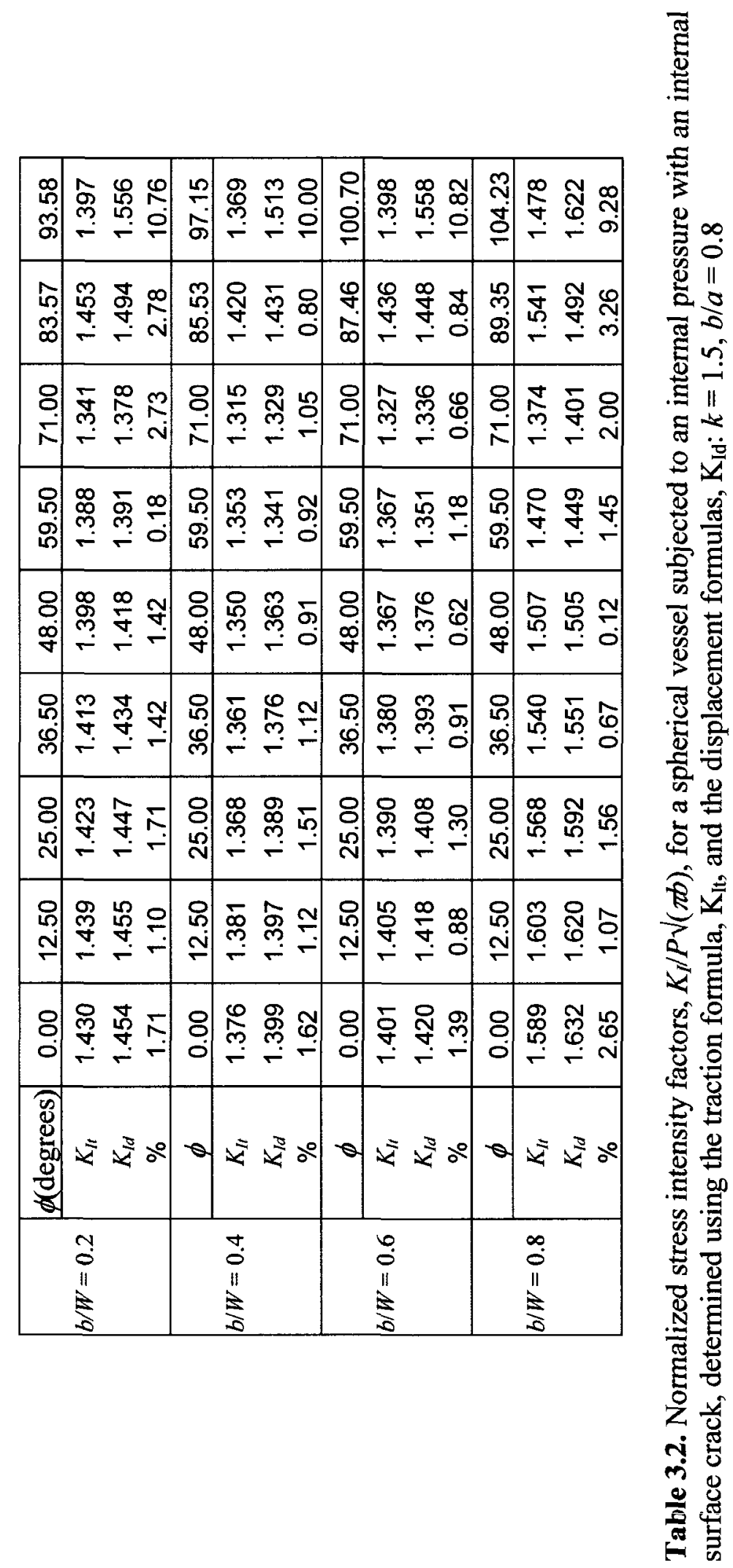




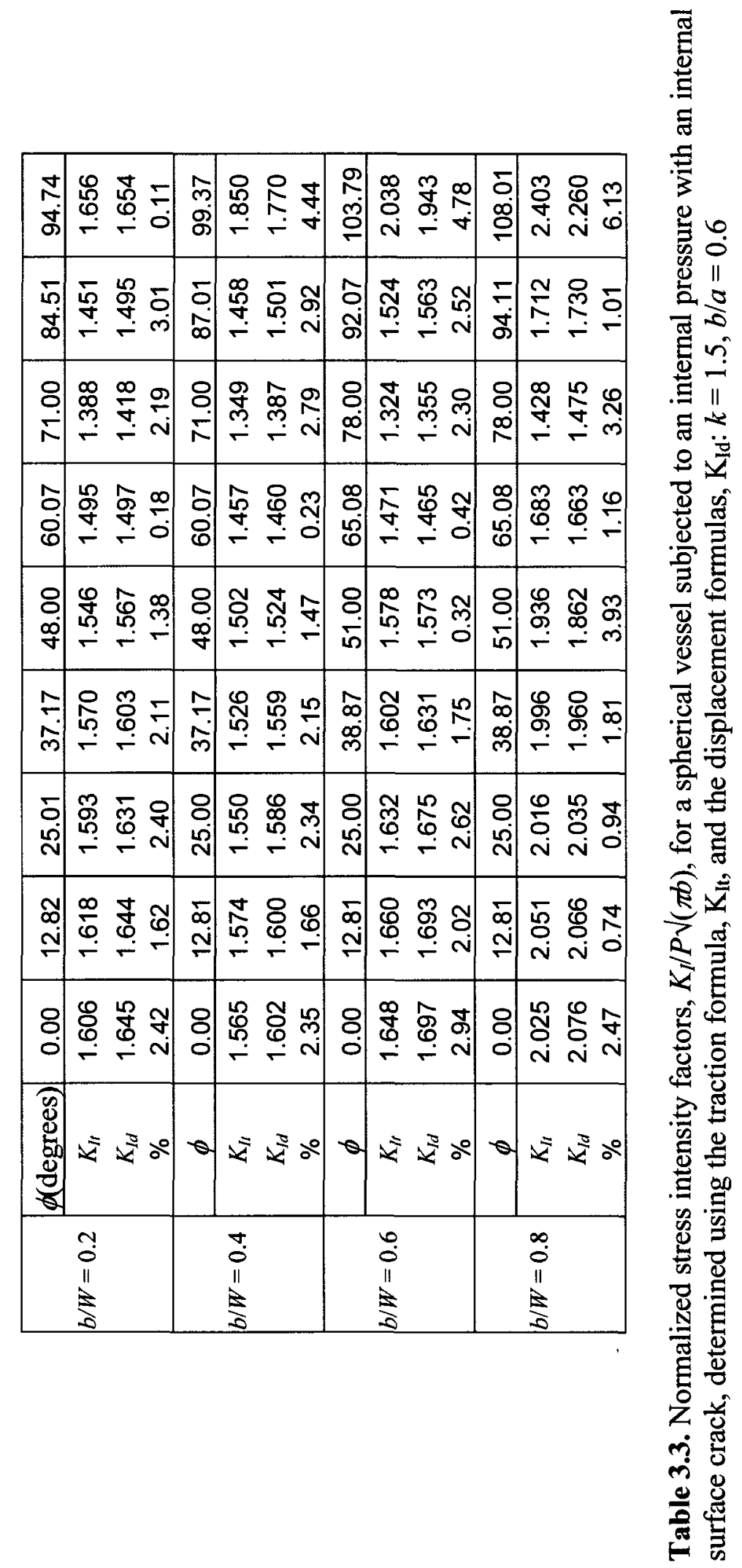




\begin{tabular}{|c|c|c|c|c|c|c|c|}
\hline $\begin{array}{l}N \\
\text { L } \\
\text { L }\end{array}$ & 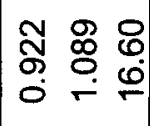 & 节 & 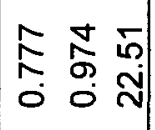 & 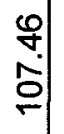 & 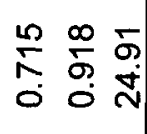 & 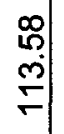 & 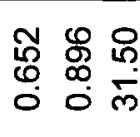 \\
\hline $\begin{array}{l}\hat{\infty} \\
\text { ஸे } \\
\infty\end{array}$ & 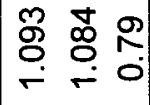 & 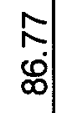 & 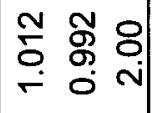 & $\begin{array}{c}\frac{n}{2} \\
8\end{array}$ & 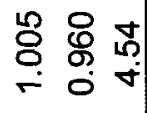 & $\begin{array}{l}9 \\
\stackrel{9}{\phi}\end{array}$ & 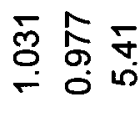 \\
\hline $\begin{array}{l}8 \\
\text { N̦}\end{array}$ & $\begin{array}{lll}\infty & \hat{m} & 0 \\
\tilde{O} & 0 & 0 \\
& 0 & 0\end{array}$ & 엄 & $\mid \begin{array}{lll}\hat{\mathscr{O}} & 0 & 0 \\
\mathscr{O} & 0 & 0 \\
0 & 0 & 0\end{array}$ & $\begin{array}{l}8 \\
\dot{+} \\
+\end{array}$ & 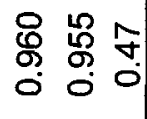 & $\begin{array}{l}8 \\
\\
0\end{array}$ & $\stackrel{0}{\circ} \stackrel{0}{\circ} \underset{0}{\circ}$ \\
\hline $\begin{array}{l}0 \\
\text { ํ. } \\
8 \\
\end{array}$ & ڤิ & $\begin{array}{l}0 \\
0 \\
0 \\
0\end{array}$ & 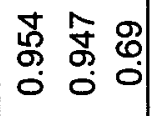 & $\begin{array}{l}8 \\
\text { ภิ }\end{array}$ & 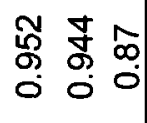 & $\begin{array}{l}8 \\
8 \\
8\end{array}$ & 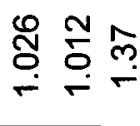 \\
\hline $\begin{array}{l}8 \\
9 \\
9\end{array}$ & 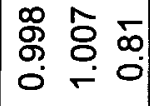 & $\begin{array}{l}8 \\
8 \\
8\end{array}$ & 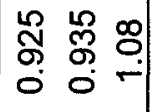 & $\begin{array}{l}8 \\
8 \\
0\end{array}$ & 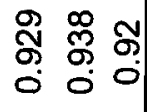 & $\begin{array}{c}8 \\
\text { กิ } \\
\text { ஸे }\end{array}$ & ָㅜㅁ \\
\hline $\begin{array}{l}8 \\
\text { n. } \\
\text { | }\end{array}$ & 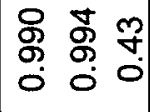 & $\begin{array}{l}8 \\
0 \\
m\end{array}$ & 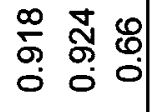 & 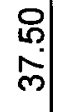 & 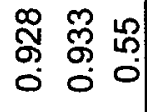 & $\begin{array}{l}0 \\
\infty \\
\infty \\
\infty\end{array}$ & స̃ \\
\hline $\begin{array}{l}8 \\
\text { กิ }\end{array}$ & 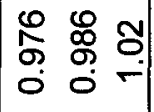 & $\begin{array}{c}8 \\
0 \\
\text { ஸी }\end{array}$ & 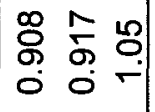 & $\begin{array}{c}8 \\
\stackrel{1}{n}\end{array}$ & 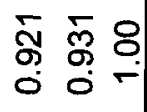 & $\begin{array}{l}8 \\
\text { ஸุ } \\
\text { N }\end{array}$ & 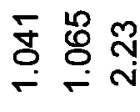 \\
\hline $\begin{array}{l}\stackrel{0}{0} \\
\stackrel{\leftrightarrow}{\sim}\end{array}$ & \begin{tabular}{lll}
$\hat{N}$ & $\bar{\infty}$ & \multirow{4}{*}{} \\
$\sigma$ & 0 \\
0 & 0 & 0
\end{tabular} & 유 & 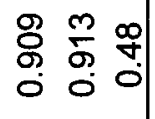 & $\begin{array}{c}\text { 오 } \\
\stackrel{N}{*}\end{array}$ & 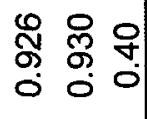 & 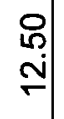 & 市 \\
\hline $\begin{array}{l}8 \\
0 \\
0\end{array}$ & 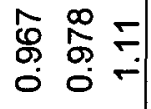 & 임 & 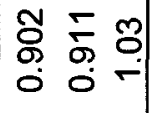 & 8 & 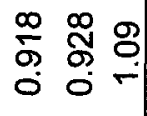 & 8 & 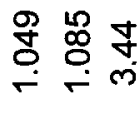 \\
\hline $\begin{array}{l}0 \\
0 \\
0 \\
0 \\
0 \\
0 \\
0\end{array}$ & $\bar{x} \times$ & of & \pm i & $\theta$ & \pm 2 & $\theta$ & 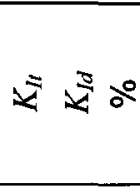 \\
\hline & 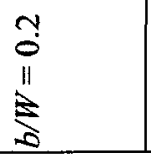 & & $\begin{array}{l}+ \\
0 \\
11 \\
0 \\
0\end{array}$ & & $\begin{array}{l}0 \\
0 \\
11 \\
0 \\
0\end{array}$ & & $\begin{array}{l}\infty \\
0 \\
11 \\
0\end{array}$ \\
\hline & & & & & & & \\
\hline
\end{tabular}




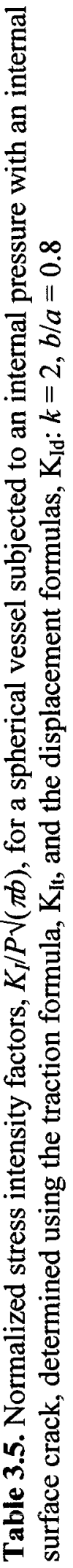




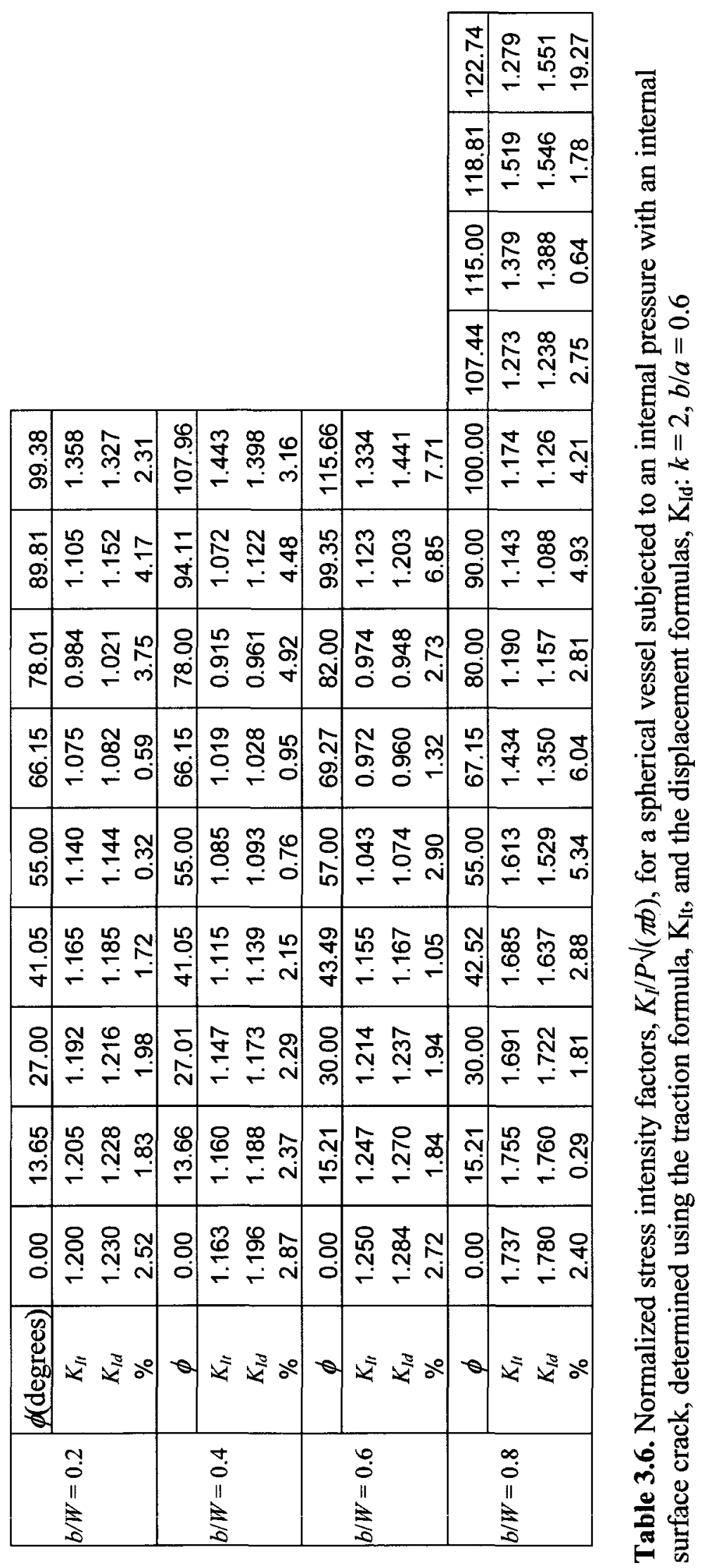




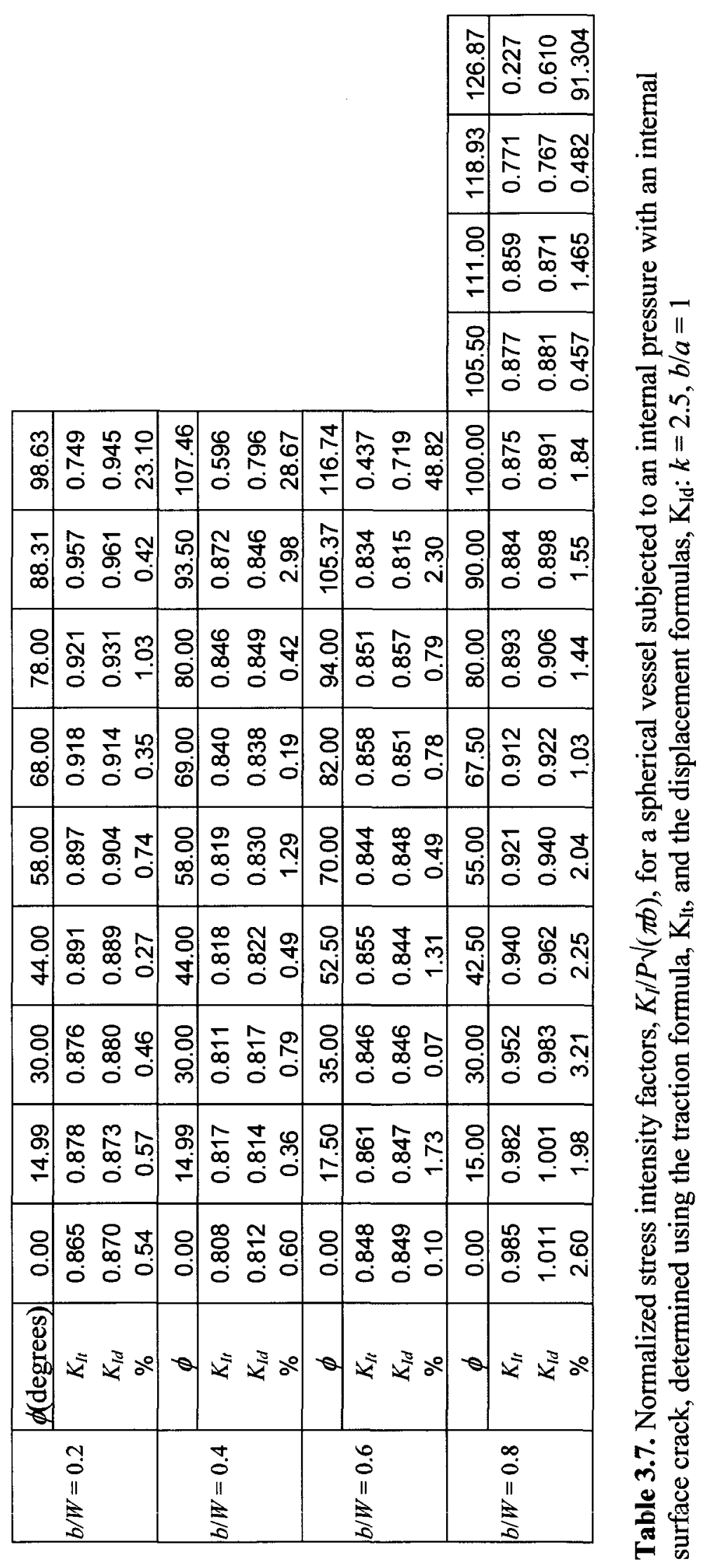




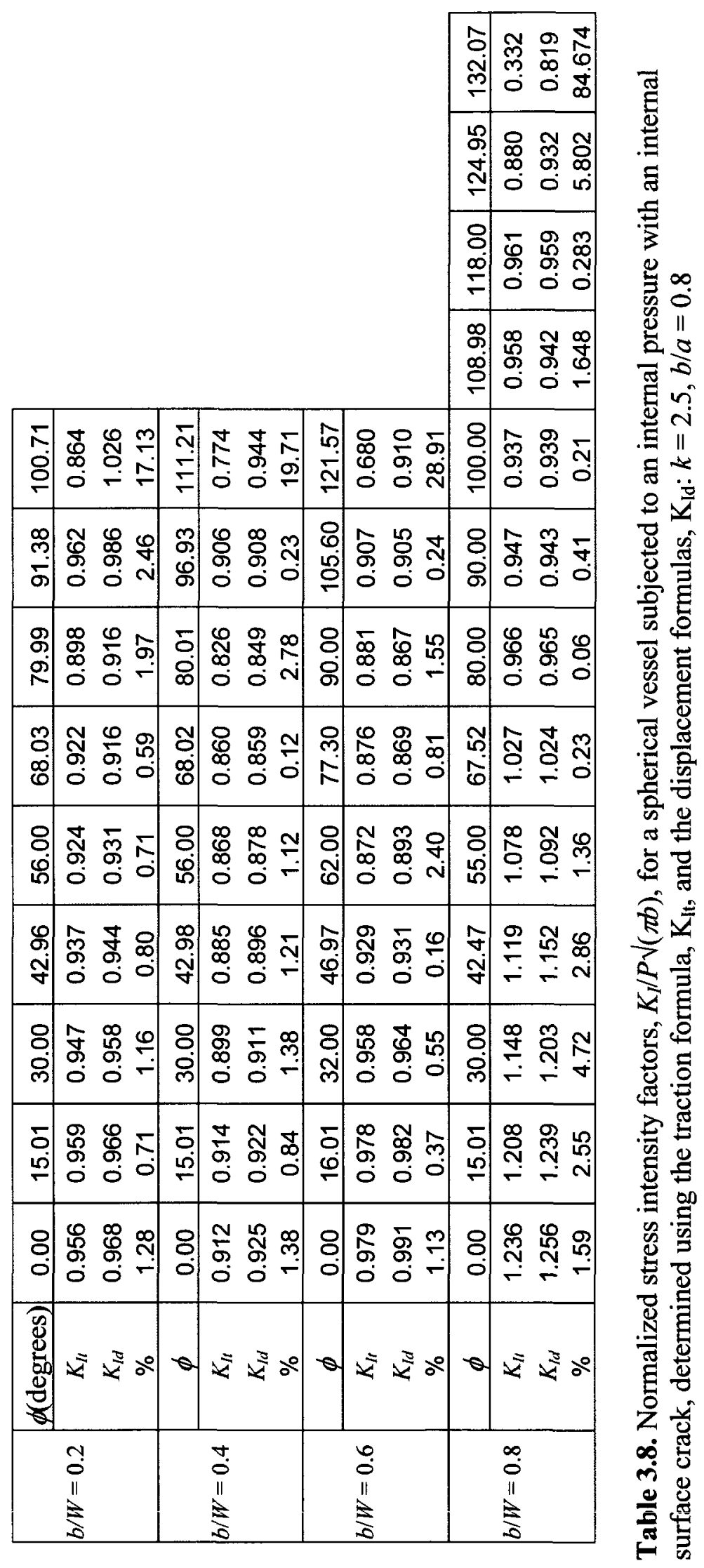




\begin{tabular}{|c|c|c|c|c|c|c|}
\hline & & & & $\begin{array}{l}\stackrel{m}{\leftarrow} \\
\stackrel{\leftrightarrow}{v}\end{array}$ & 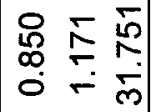 & \\
\hline & & & & $\begin{array}{l}\mathcal{N} \\
\stackrel{\mathfrak{S}}{\leftarrow}\end{array}$ & 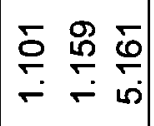 & \\
\hline & & & & \begin{tabular}{c}
8 \\
8 \\
$i \infty$ \\
\hdashline
\end{tabular} & 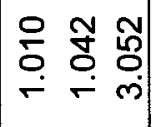 & \\
\hline & & & & 竎 & 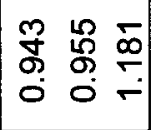 & \\
\hline $\begin{array}{l}9 \\
\text { \%े } \\
\stackrel{\circ}{\circ}\end{array}$ & 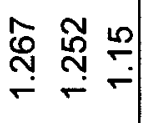 & $\begin{array}{l}\mathscr{E} \\
\stackrel{5}{ } \\
\square\end{array}$ & 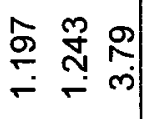 & $\begin{array}{l}8 \\
8 \\
8 \\
\end{array}$ & 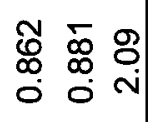 & \\
\hline $\begin{array}{l}\text { ภे } \\
\text { ภ่ }\end{array}$ & 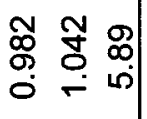 & $\begin{array}{l}\mathbf{N} \\
8 \\
\circ\end{array}$ & 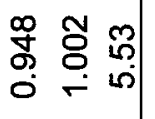 & $\begin{array}{l}\text { ৪. } \\
\text { த. }\end{array}$ & 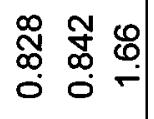 & \\
\hline $\begin{array}{l}8 \\
8 \\
8 \\
\infty\end{array}$ & 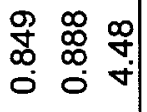 & $\begin{array}{l}8 \\
10 \\
\infty\end{array}$ & $\begin{array}{lll}\infty & \mathscr{8} & \\
\mathfrak{N} & \delta & 0 \\
0 & 0 & 0 \\
0 & 0 & 0\end{array}$ & $\begin{array}{l}8 \\
8 \\
8 \\
0\end{array}$ & $\begin{array}{lll}5 & 0 & 0 \\
\infty & \infty & 0 \\
0 & 0 & 0 \\
0 & 0\end{array} \mid$ & \\
\hline $\begin{array}{l}\mathscr{1} \\
\infty \\
0\end{array}$ & 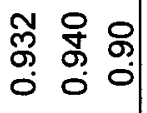 & 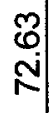 & $\begin{array}{lll}8 & 8 & 1 \\
0 & 0 & 0 \\
0 & 0 & -\end{array}$ & 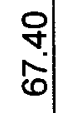 & 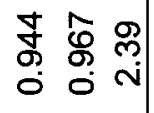 & \\
\hline $\begin{array}{l}\text { S } \\
\text { in }\end{array}$ & 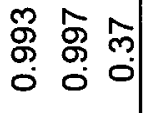 & $\begin{array}{l}8 \\
8 \\
8\end{array}$ & 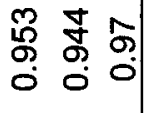 & $\begin{array}{l}8 \\
8 \\
5 \\
6\end{array}$ & 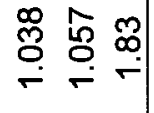 & \\
\hline $\begin{array}{l}\bar{\sigma} \\
\dot{\gamma} \\
\forall\end{array}$ & 융 웜 윰 & $\begin{array}{l}\infty \\
\infty \\
\dot{j}\end{array}$ & 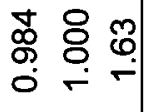 & 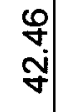 & 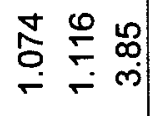 & \\
\hline $\begin{array}{l}8 \\
\text { : } \\
\text {. }\end{array}$ & 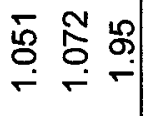 & $\begin{array}{l}8 \\
\text { : } \\
\text { ఏ. }\end{array}$ & 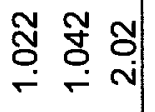 & 염 & 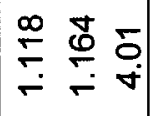 & \\
\hline$\frac{m}{60}$ & 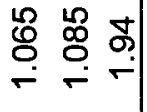 & $\frac{m}{20}$ & 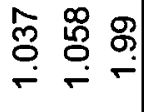 & $\frac{m}{i n}$ & 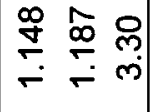 & \\
\hline $\begin{array}{l}8 \\
0 \\
0\end{array}$ & 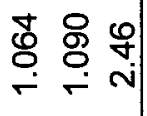 & 웅 & 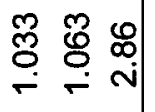 & 잉 & 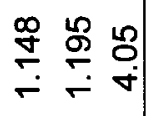 & \\
\hline $\begin{array}{l}0 \\
0 \\
0 \\
0 \\
0 \\
0 \\
0 \\
0\end{array}$ & 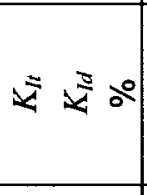 & $\theta$ & $\sqrt{2}<0$ & $\theta$ & $\bar{\nu}$ & \\
\hline & $\begin{array}{l}3 \\
0 \\
11 \\
\vdots \\
\vdots\end{array}$ & & $\begin{array}{l}+ \\
0 \\
11 \\
0 \\
0 \\
0\end{array}$ & & $\begin{array}{l}0 \\
0 \\
0 \\
11 \\
0 \\
0\end{array}$ & \\
\hline
\end{tabular}




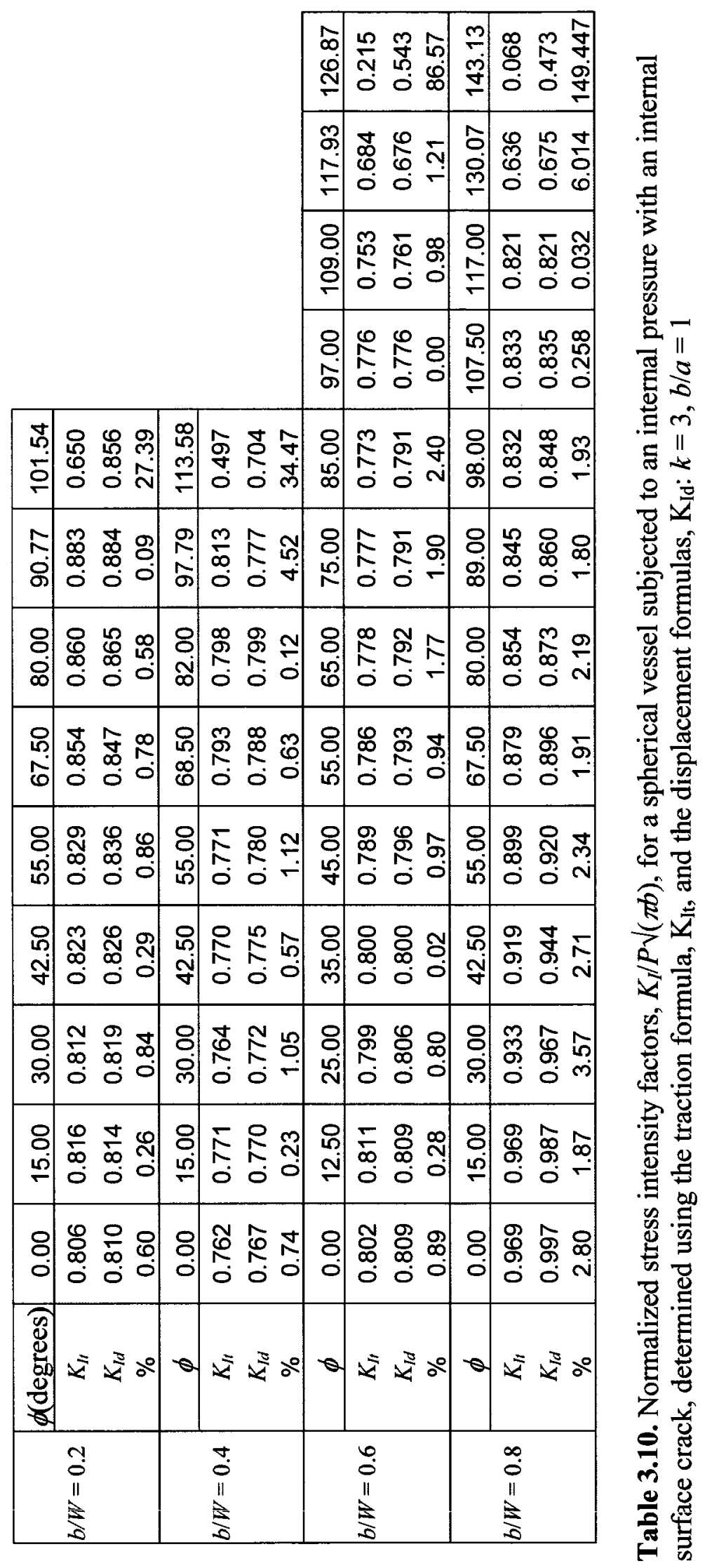




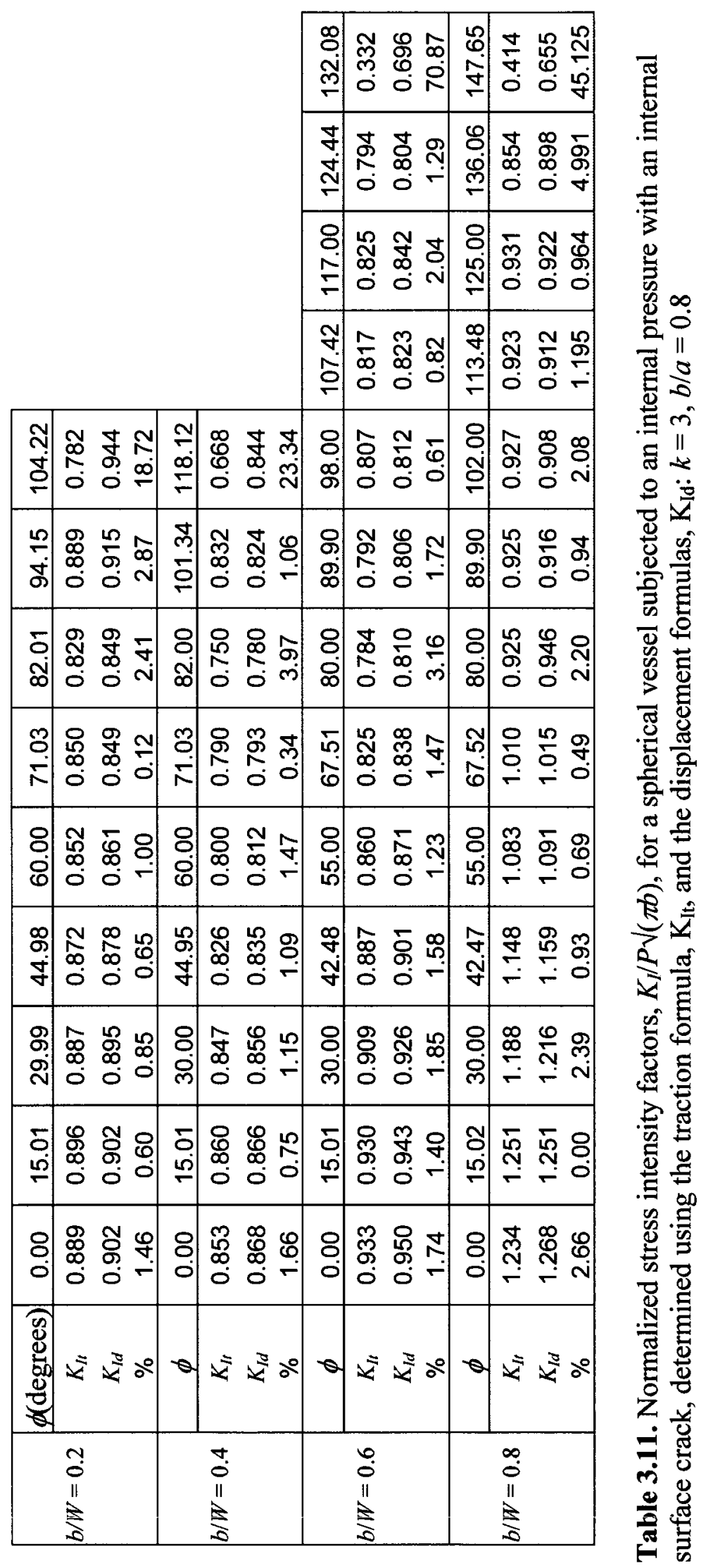




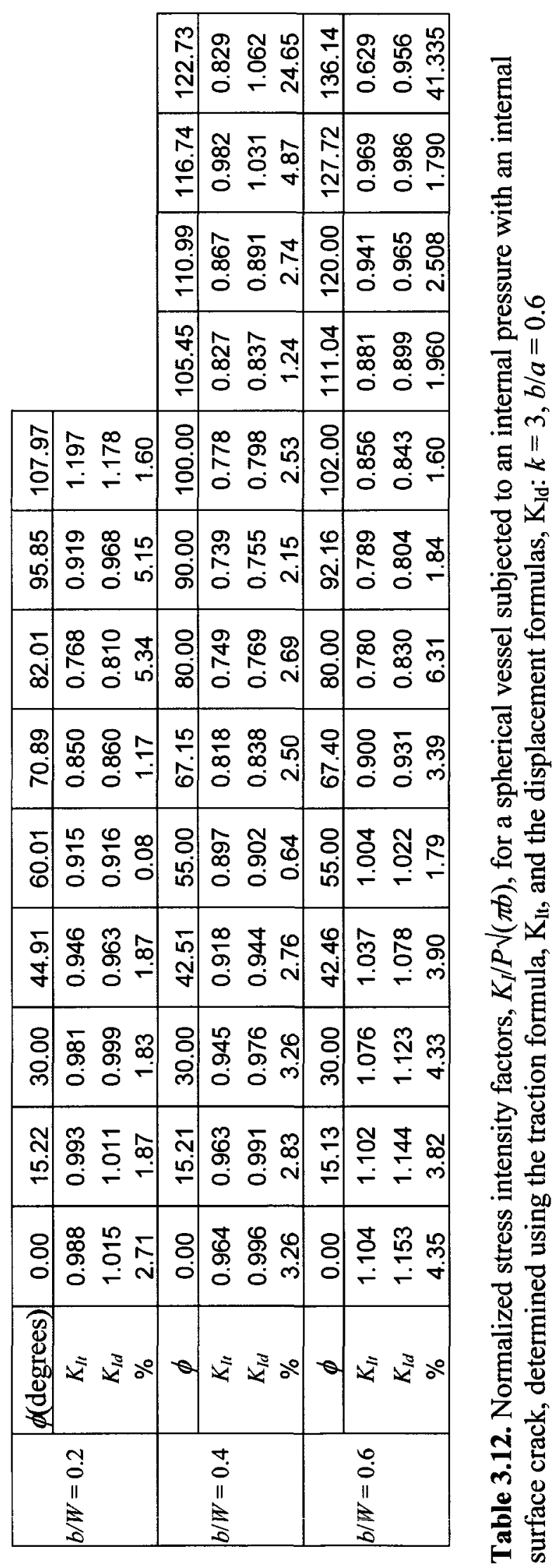




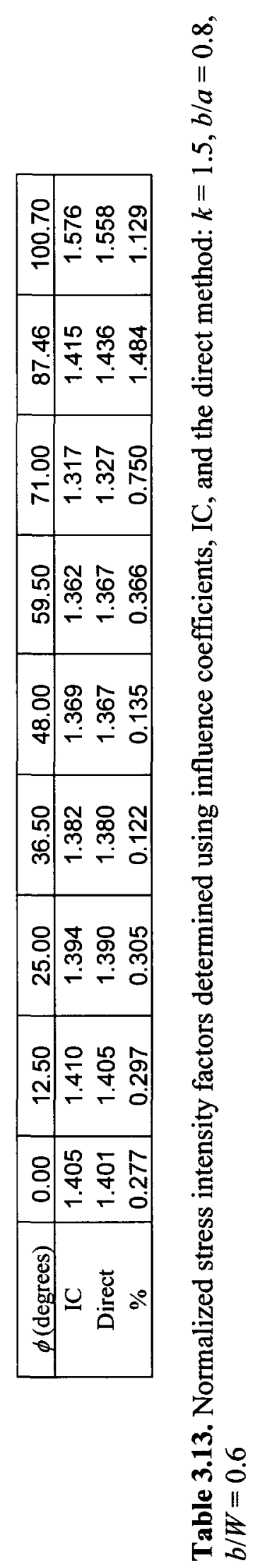




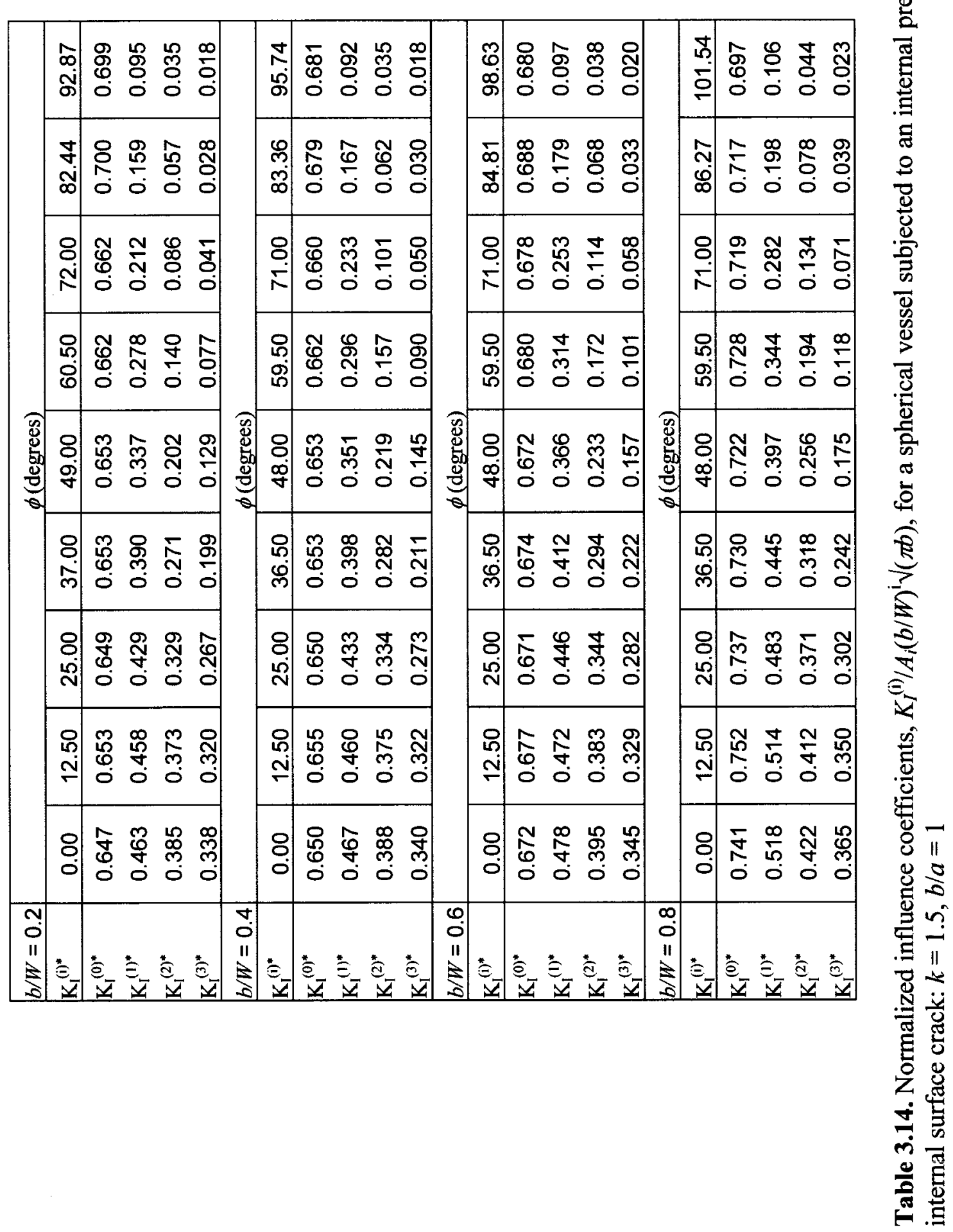




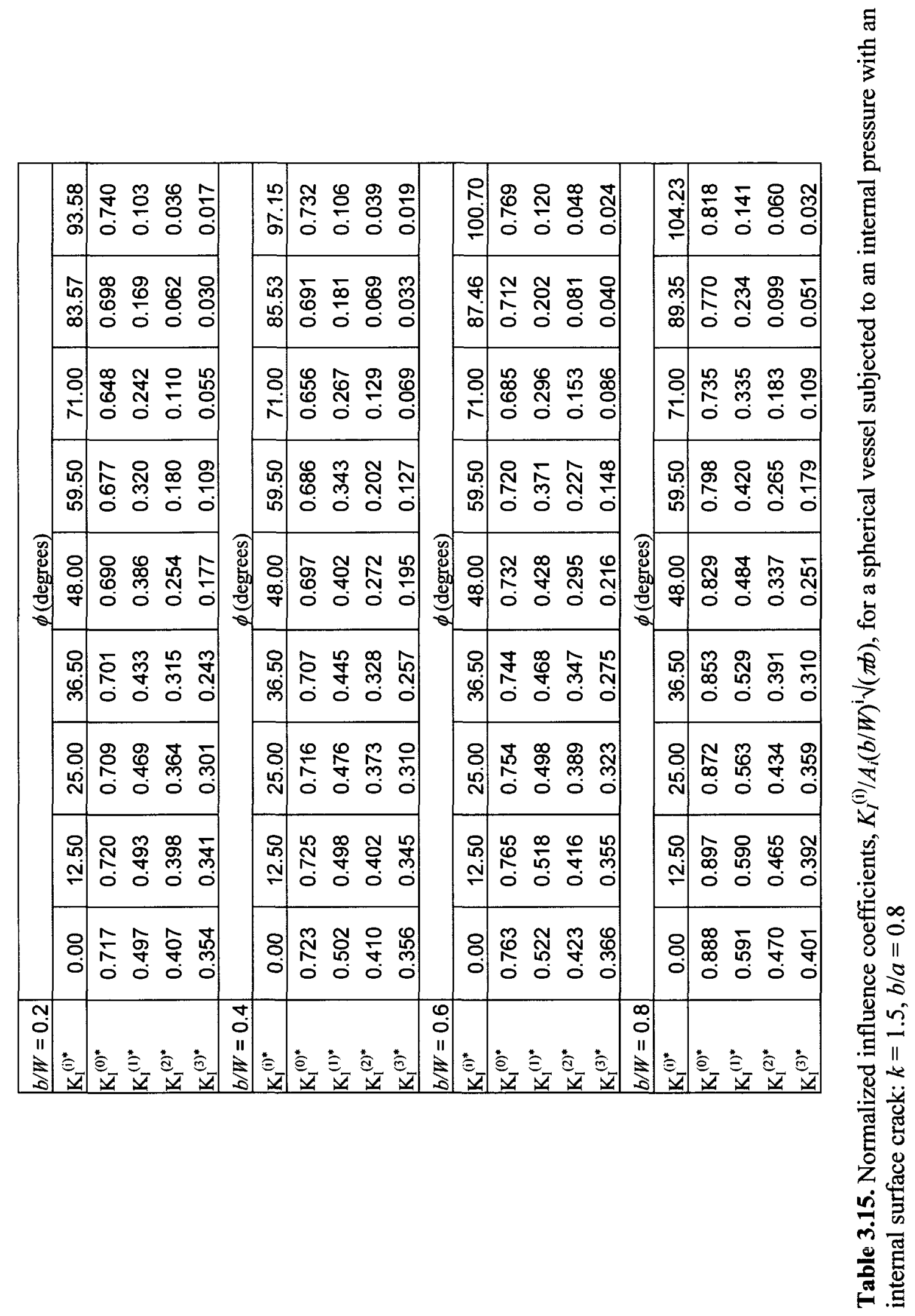




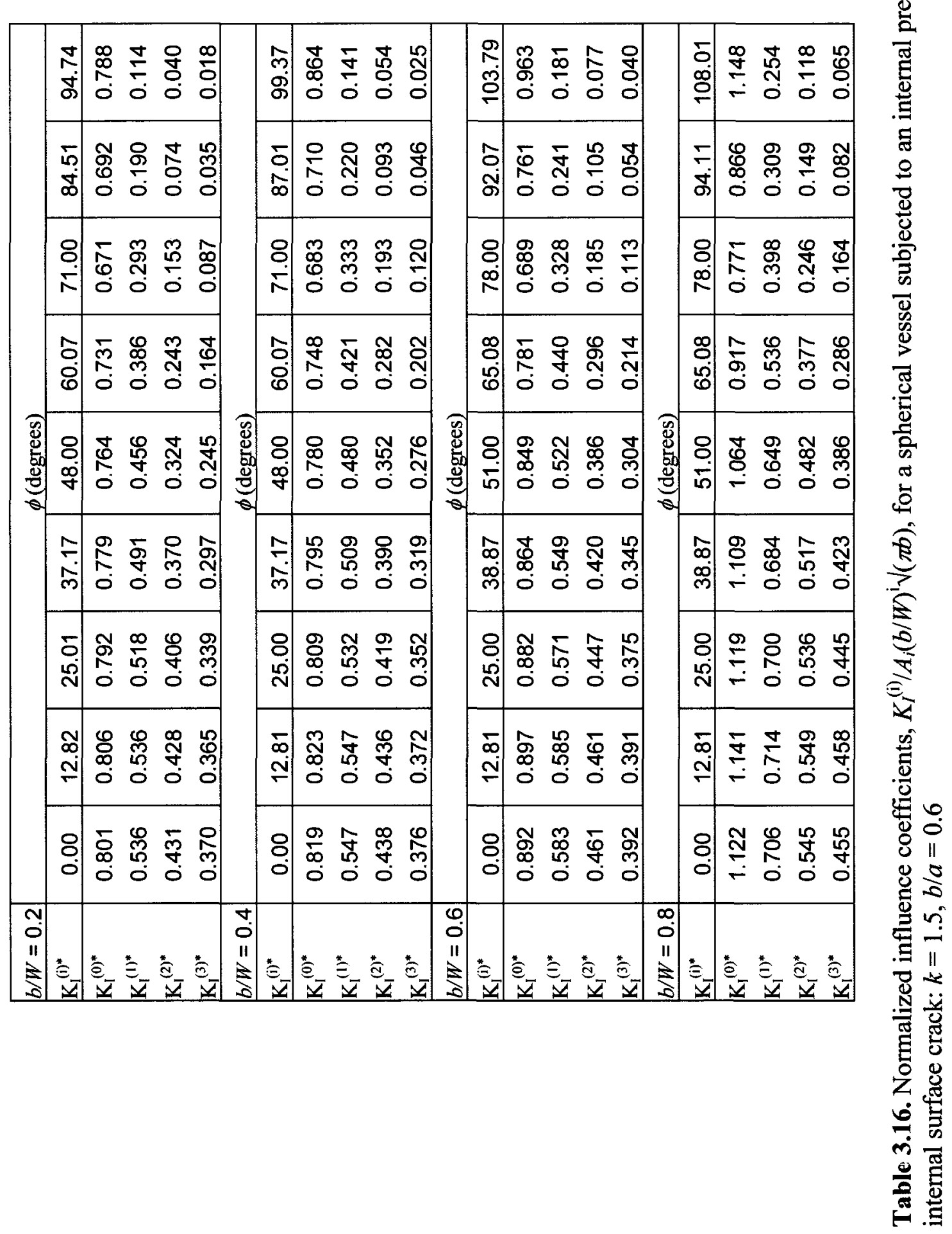




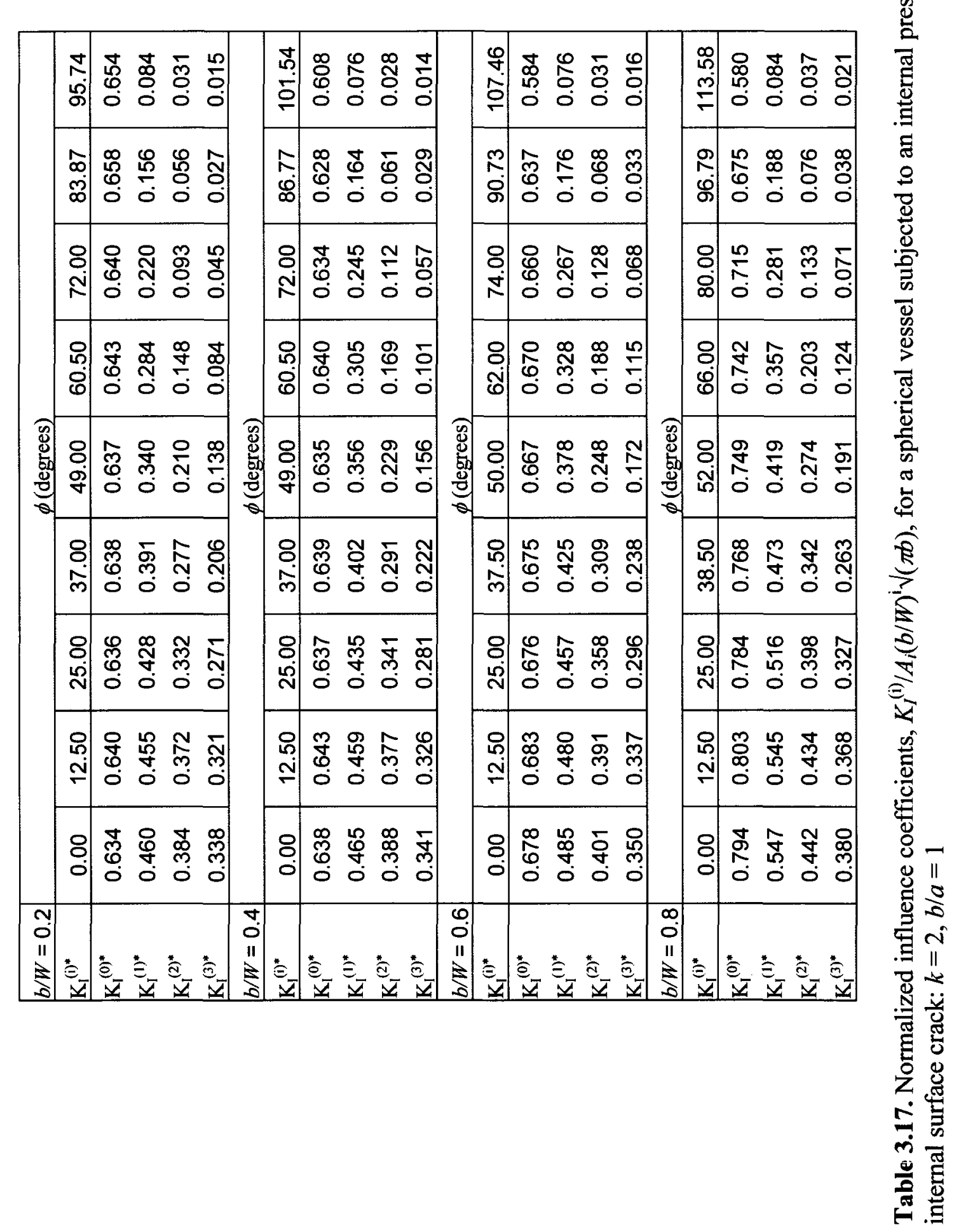




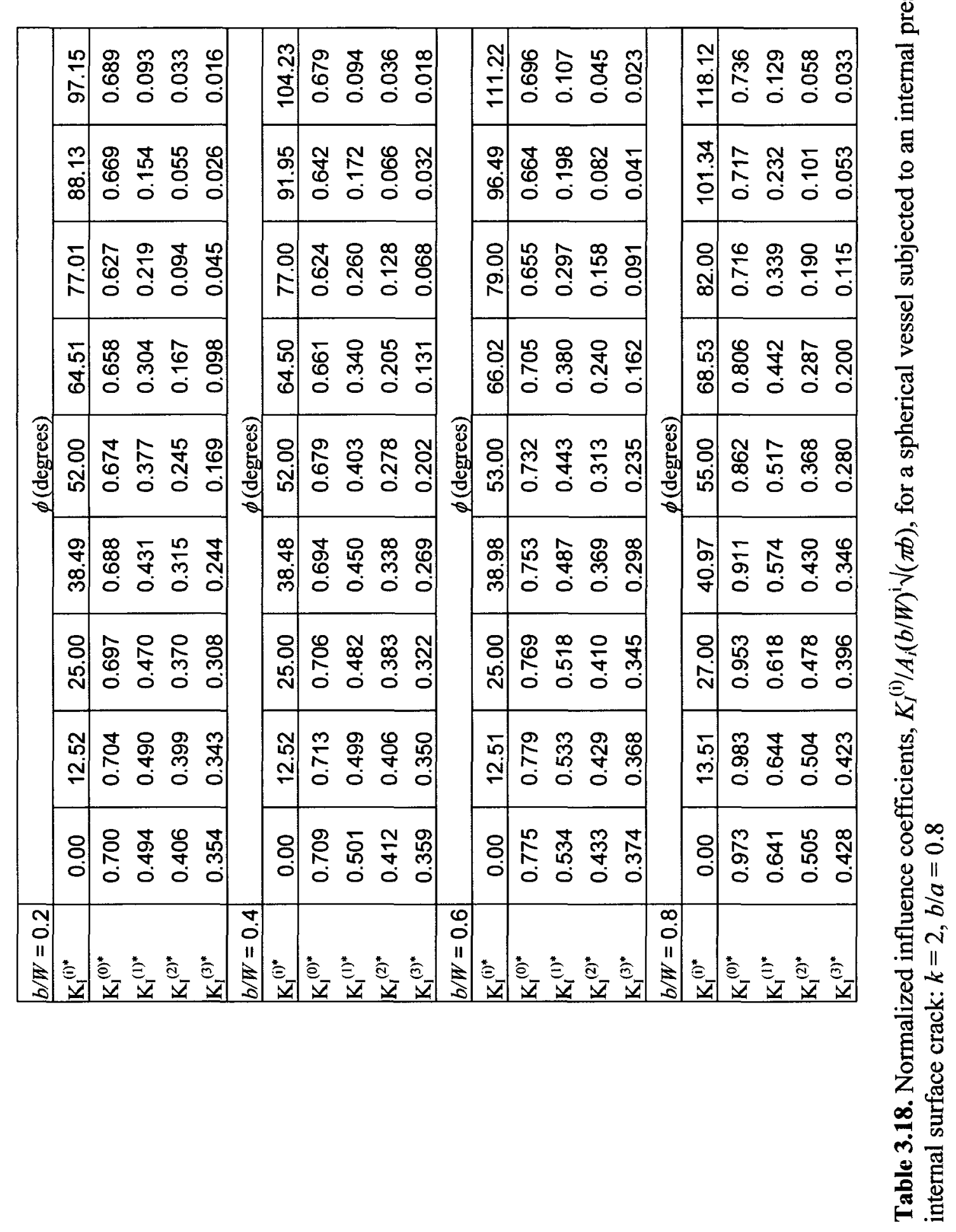




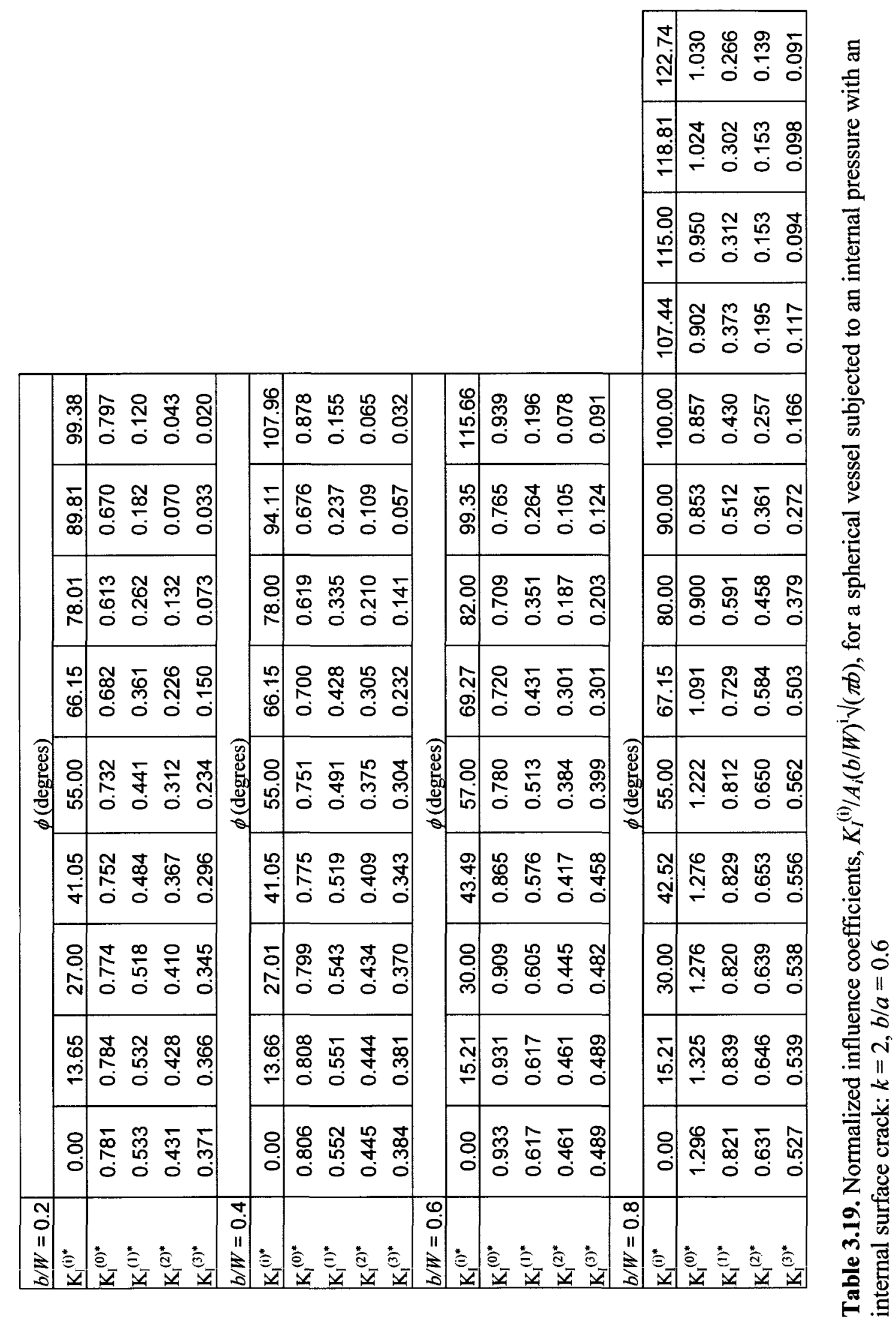




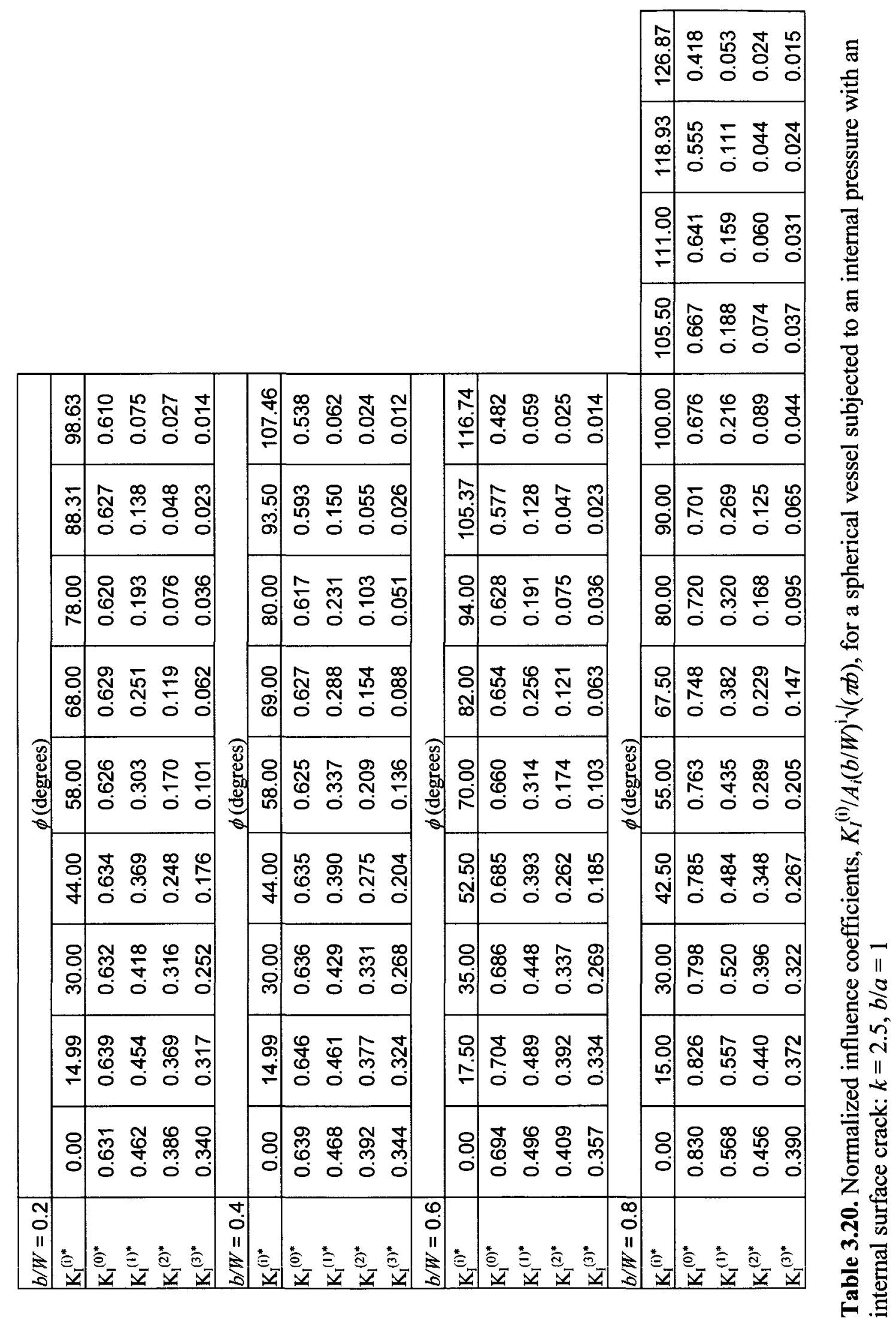




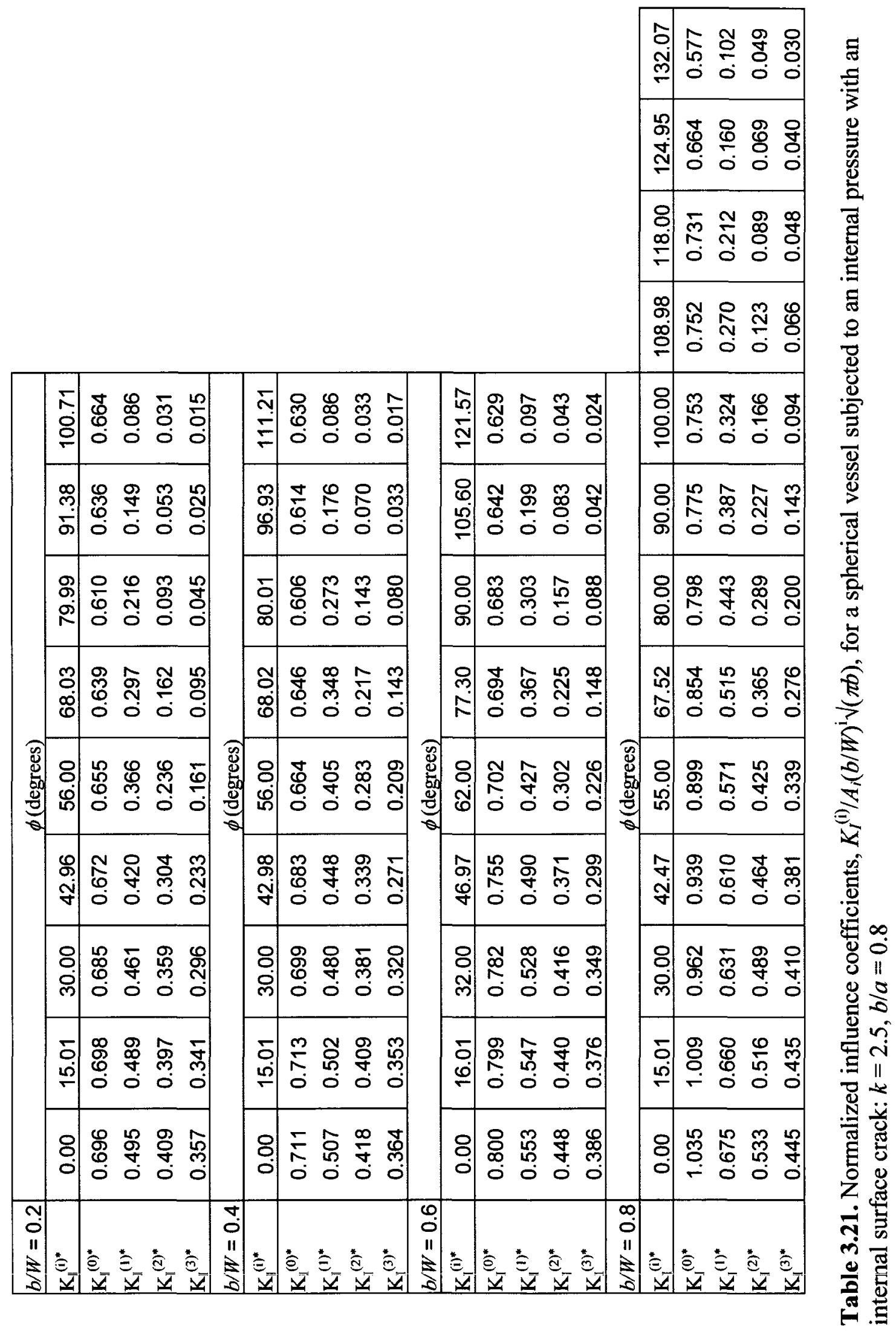




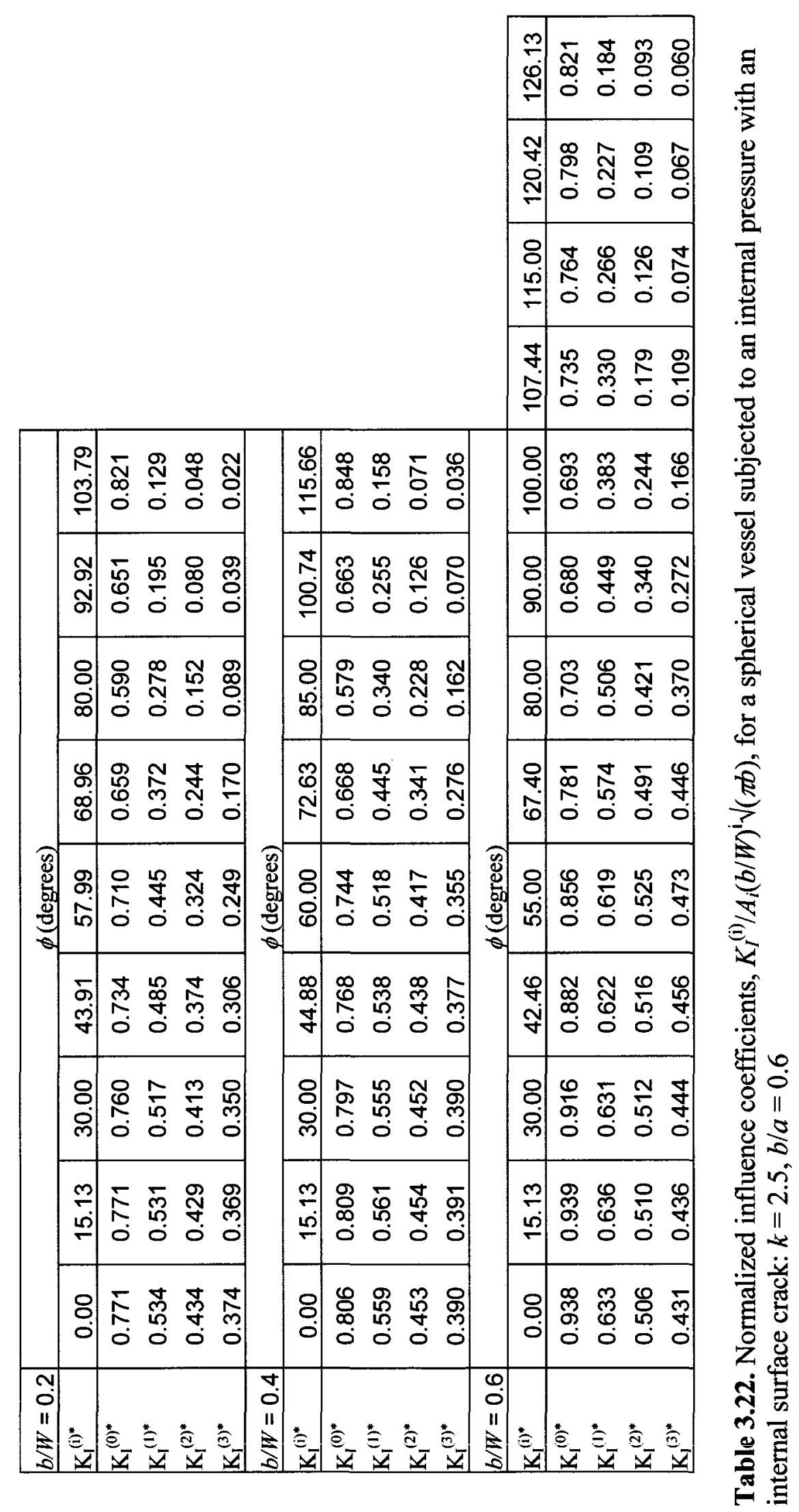




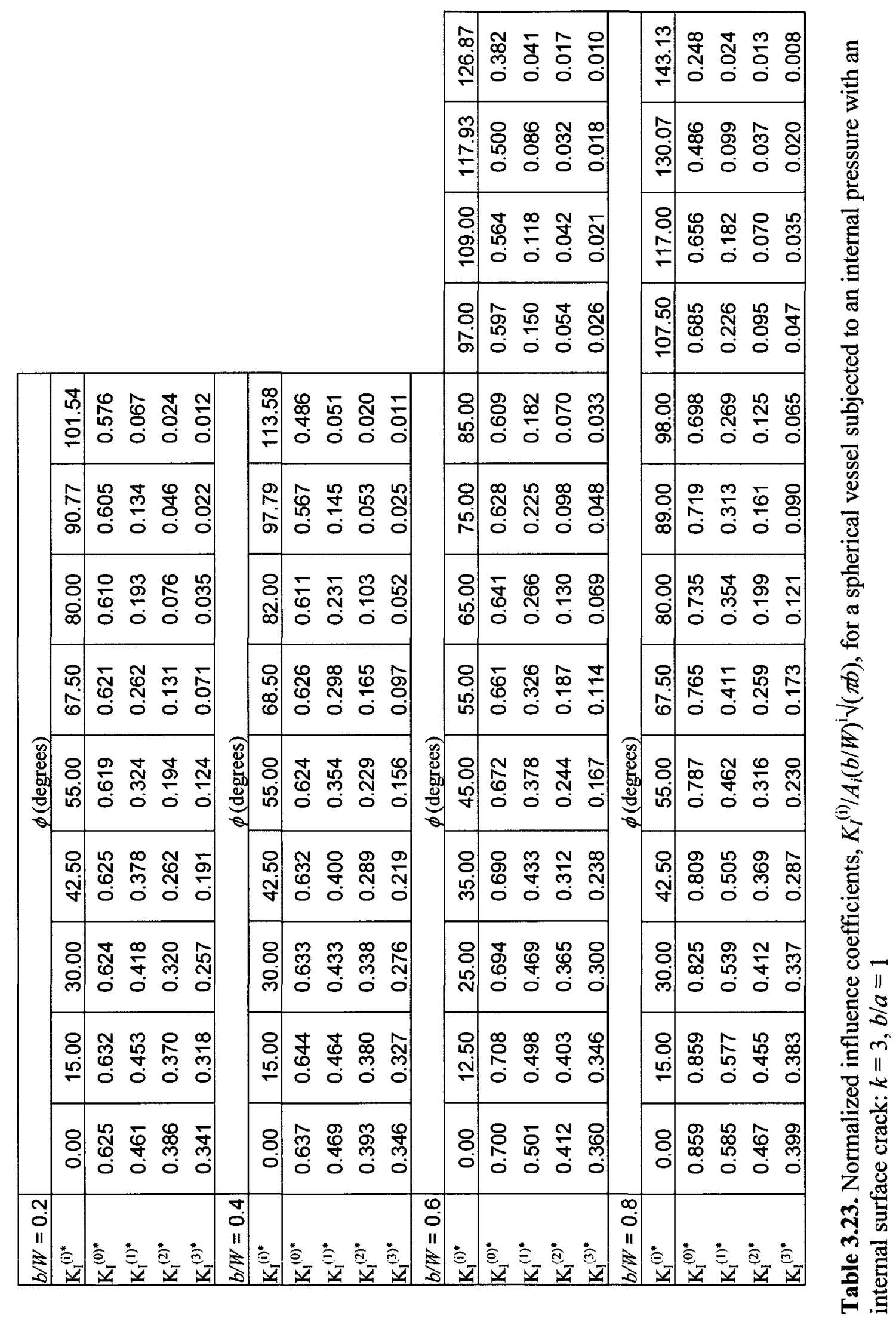




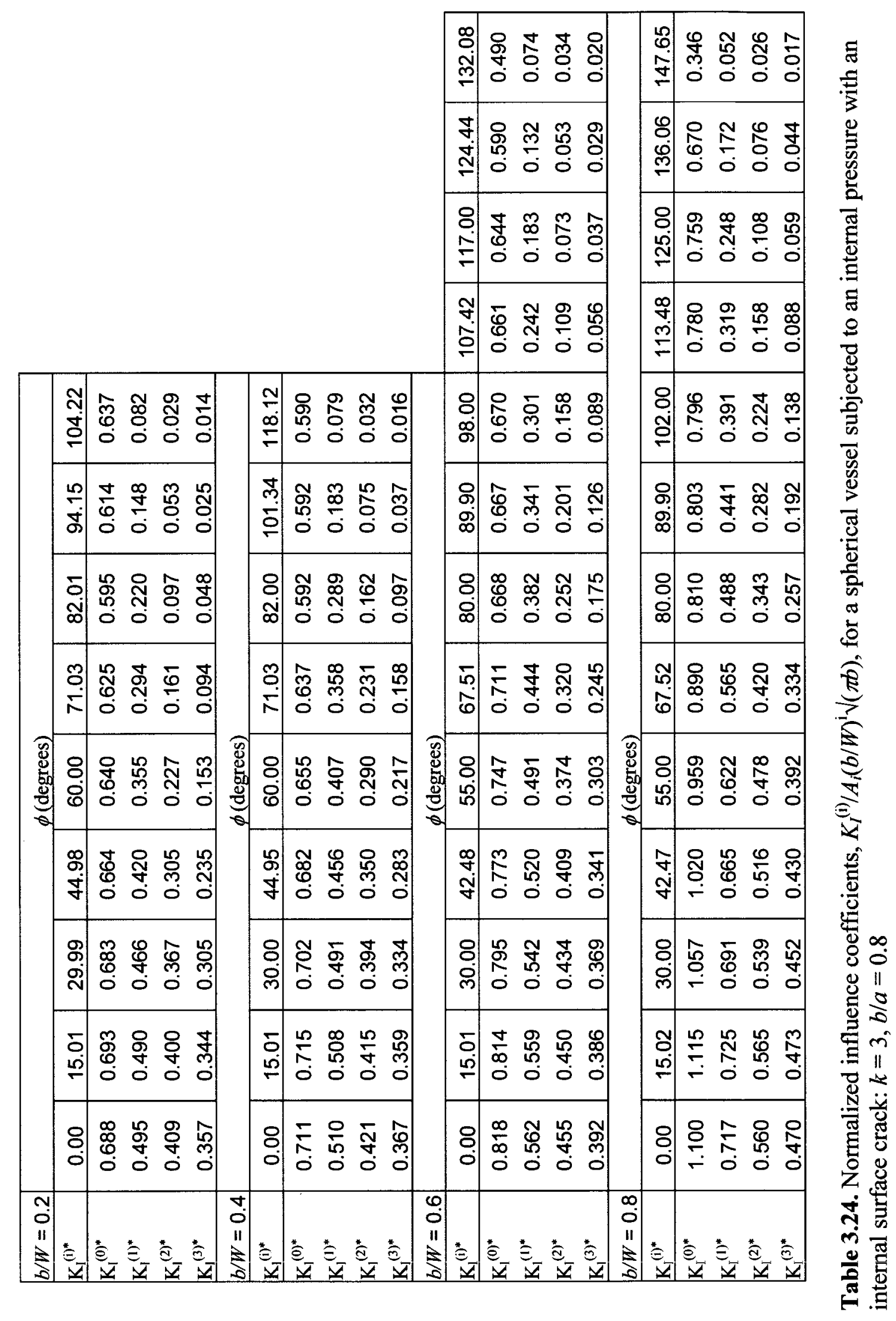




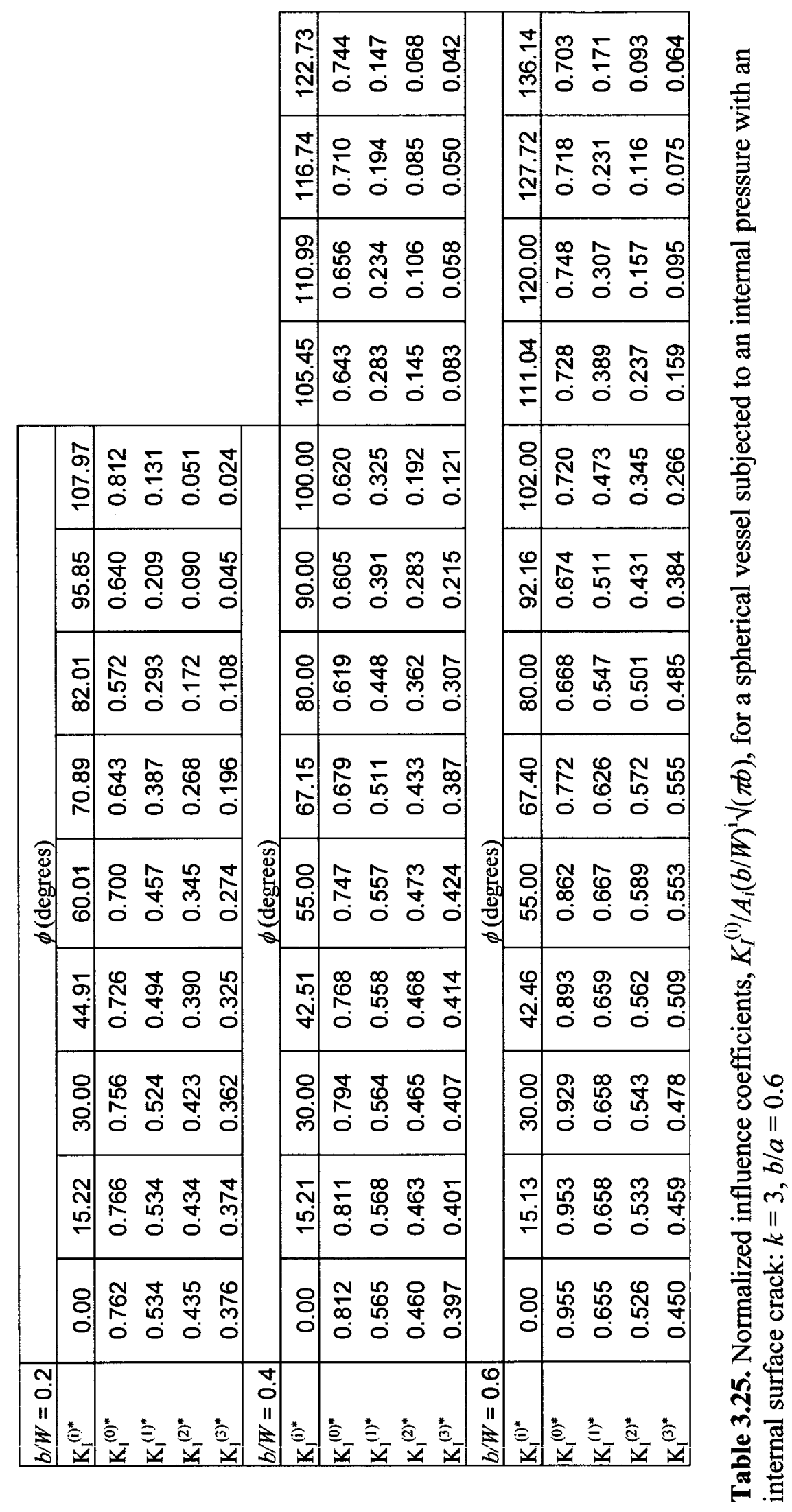




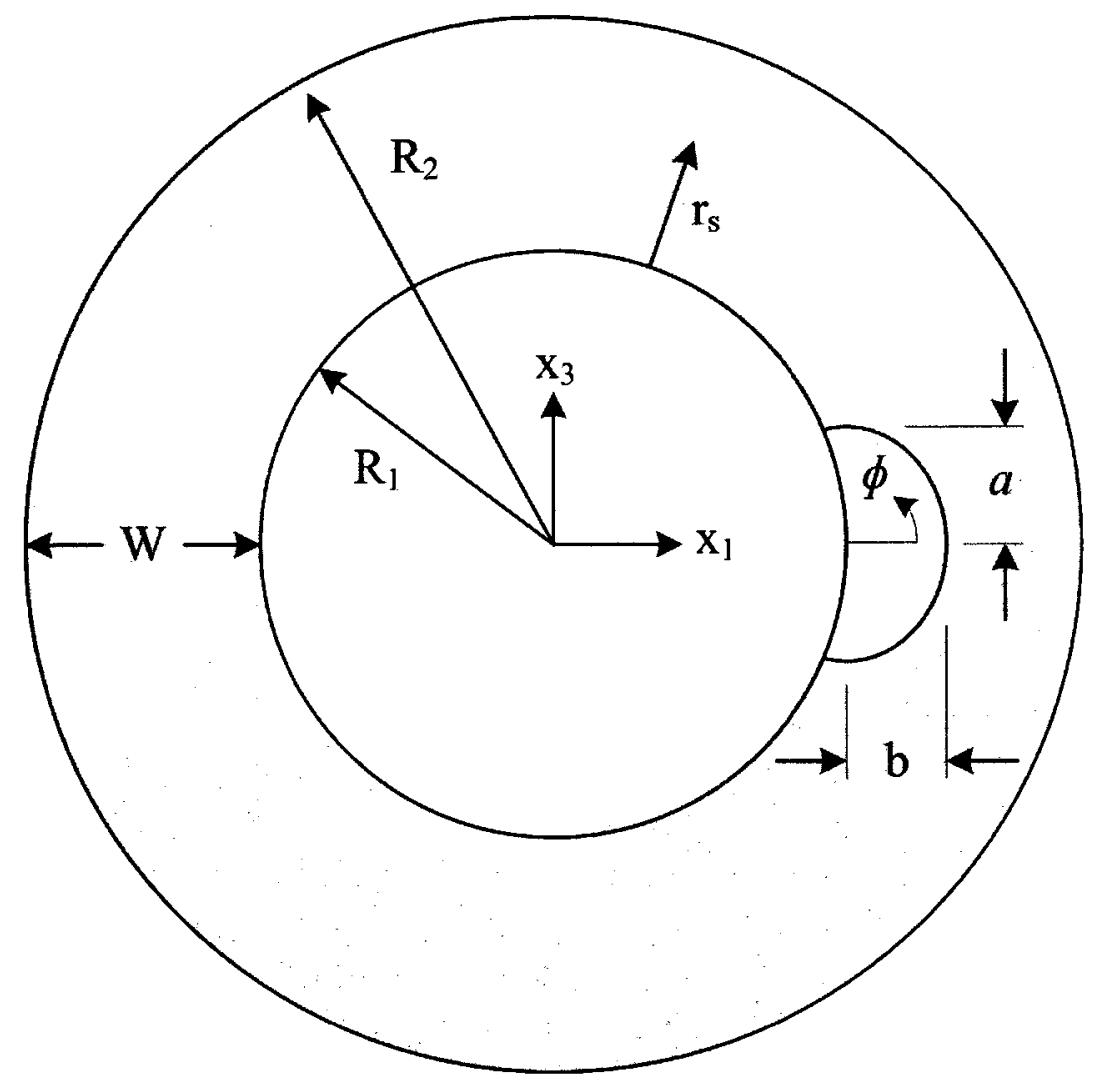

Figure 3.1. Diametrical section of a spherical vessel with an internal surface crack. 


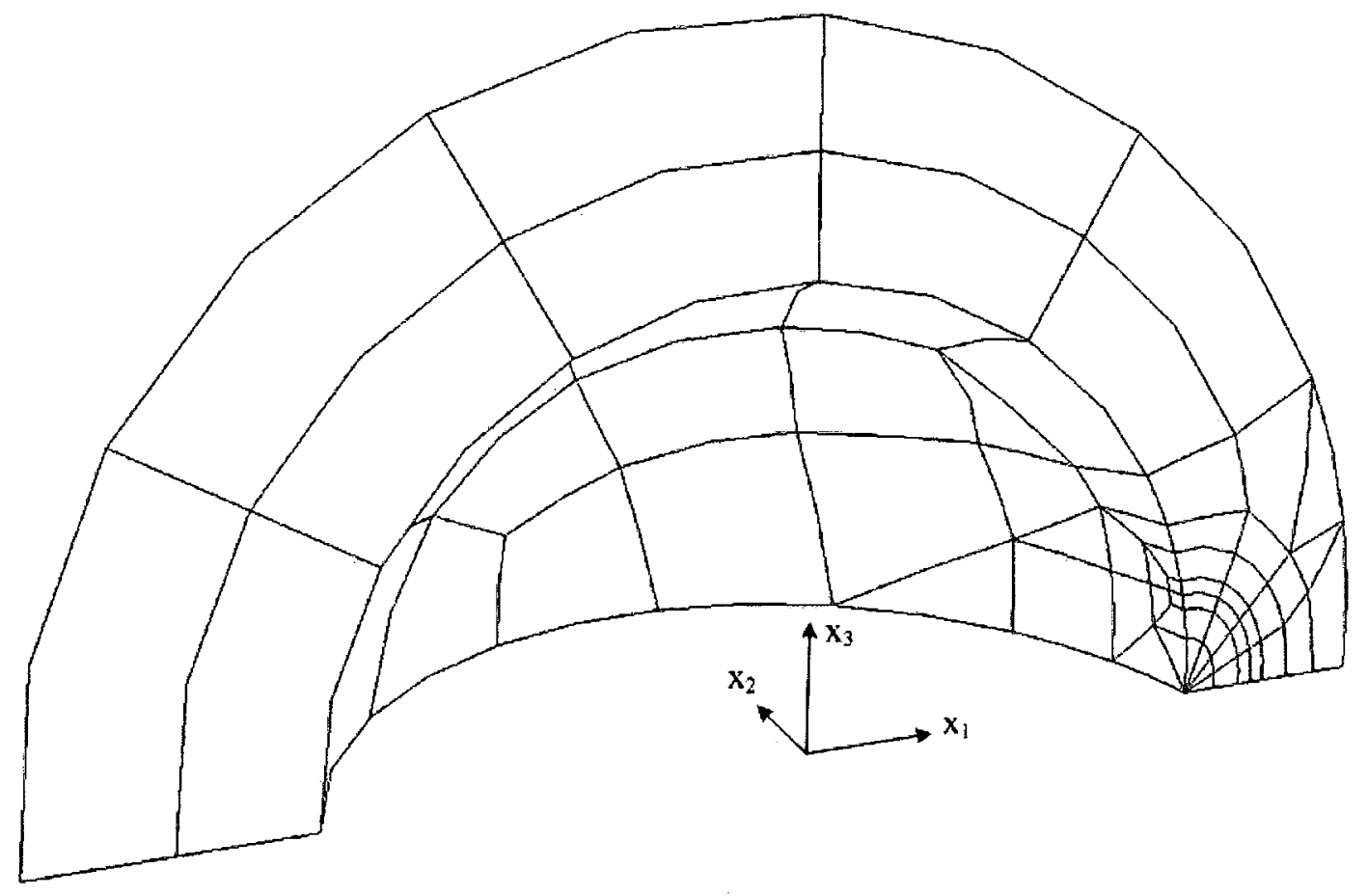

Figure 3.2. Three-dimensional BEM mesh for a spherical vessel with an internal surface crack: $\mathrm{k}=1.5, \mathrm{~b} / \mathrm{a}=0.8, \mathrm{~b} / \mathrm{W}=0.4$ 


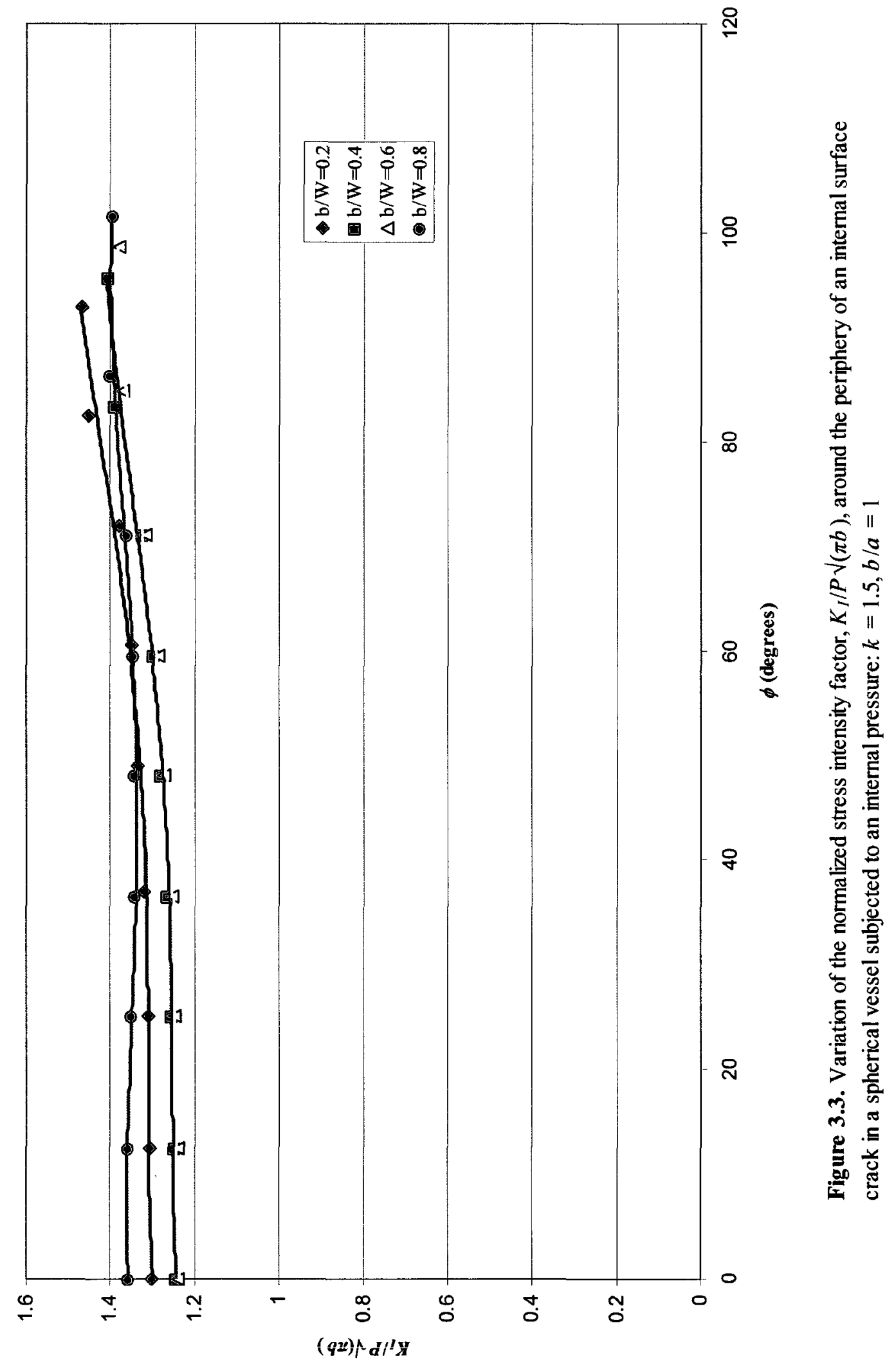




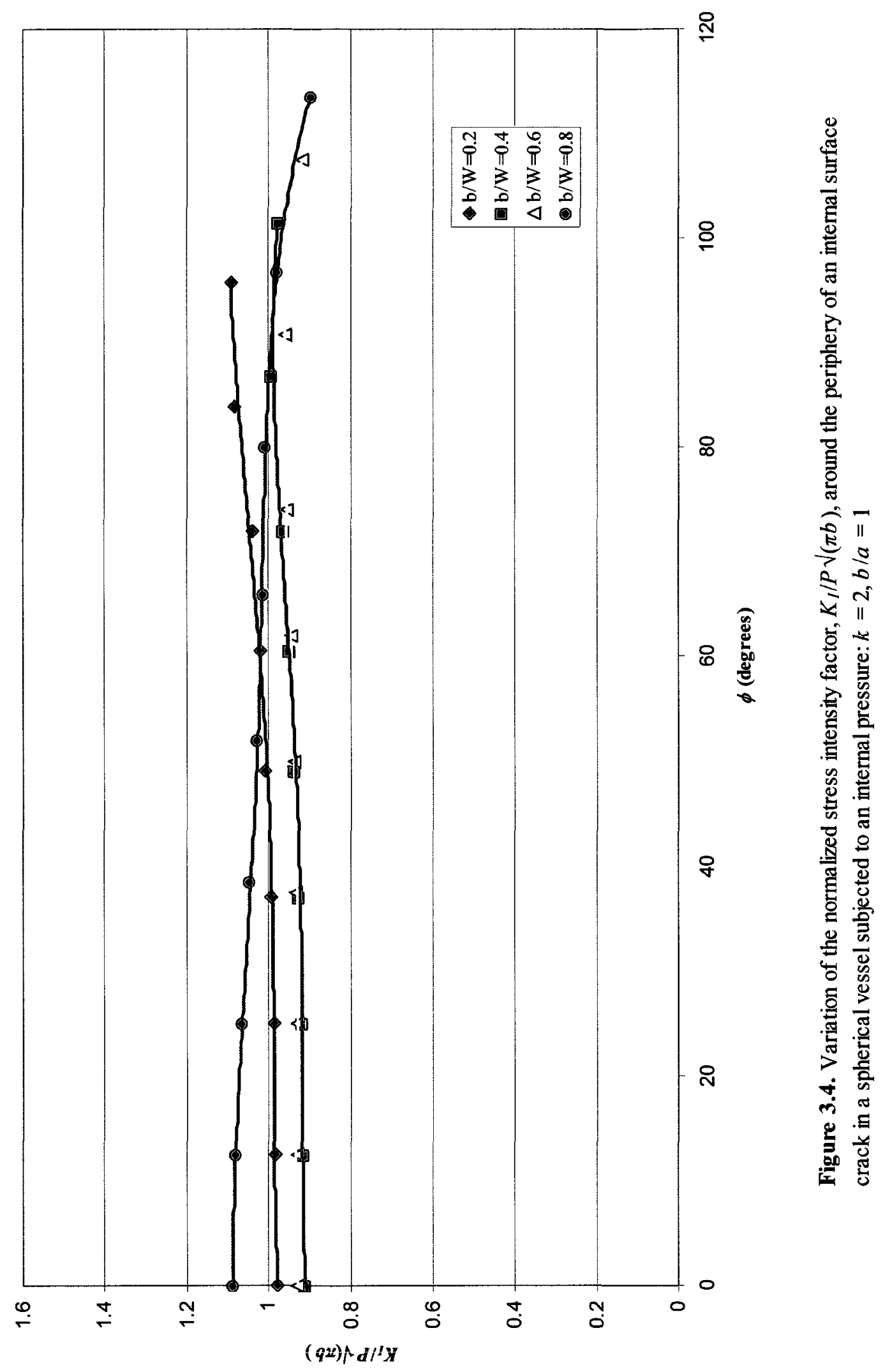




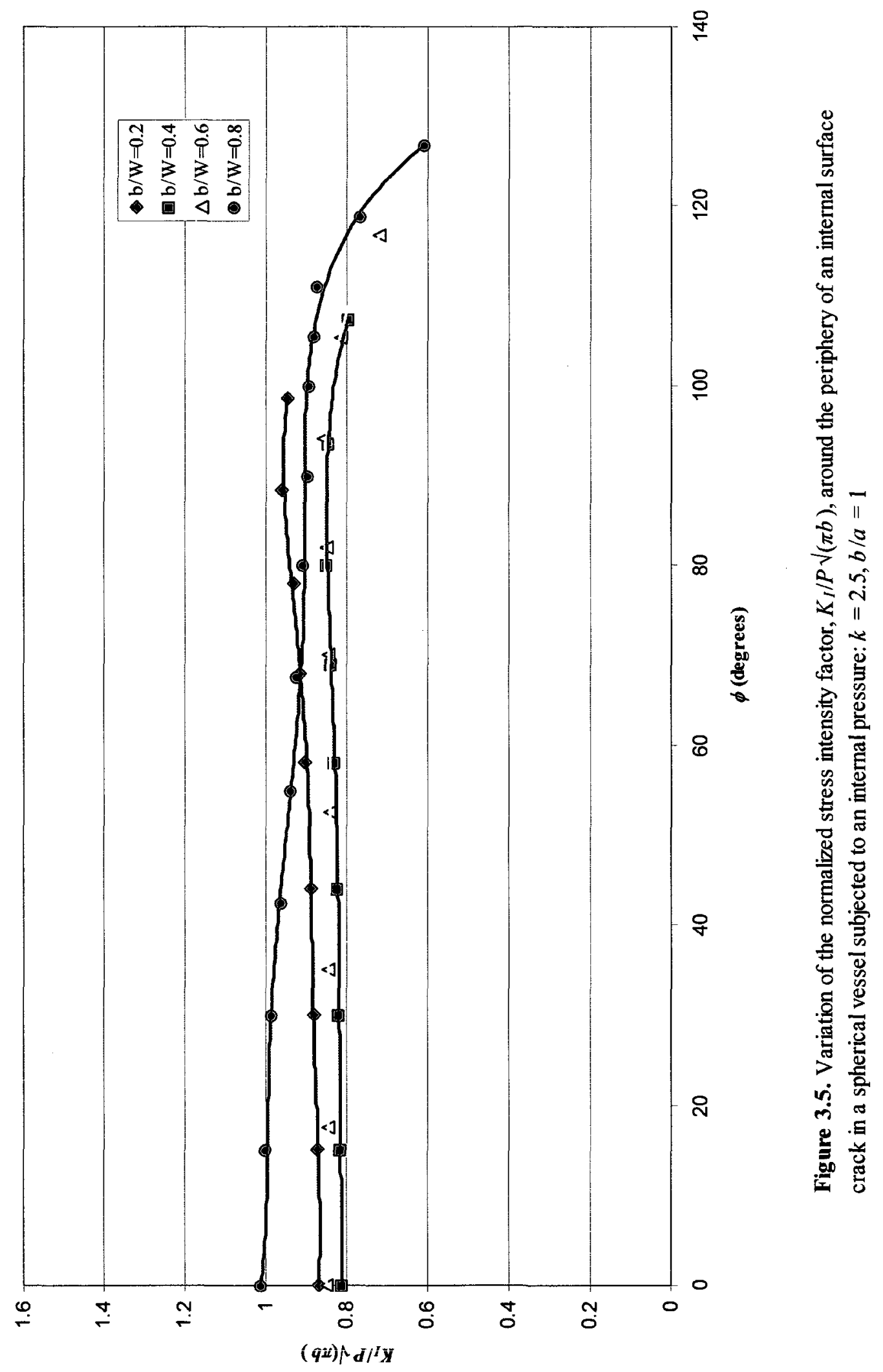




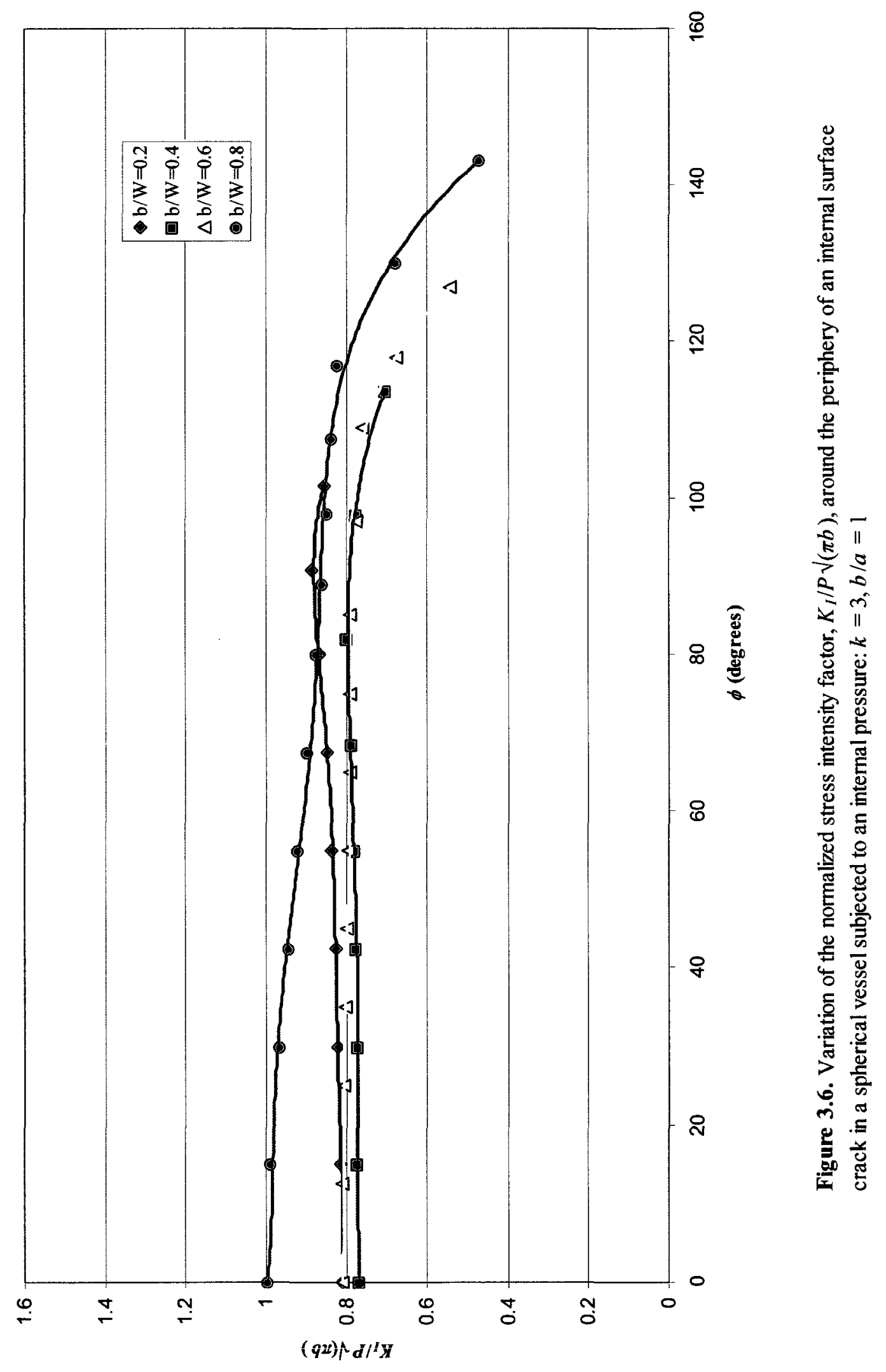




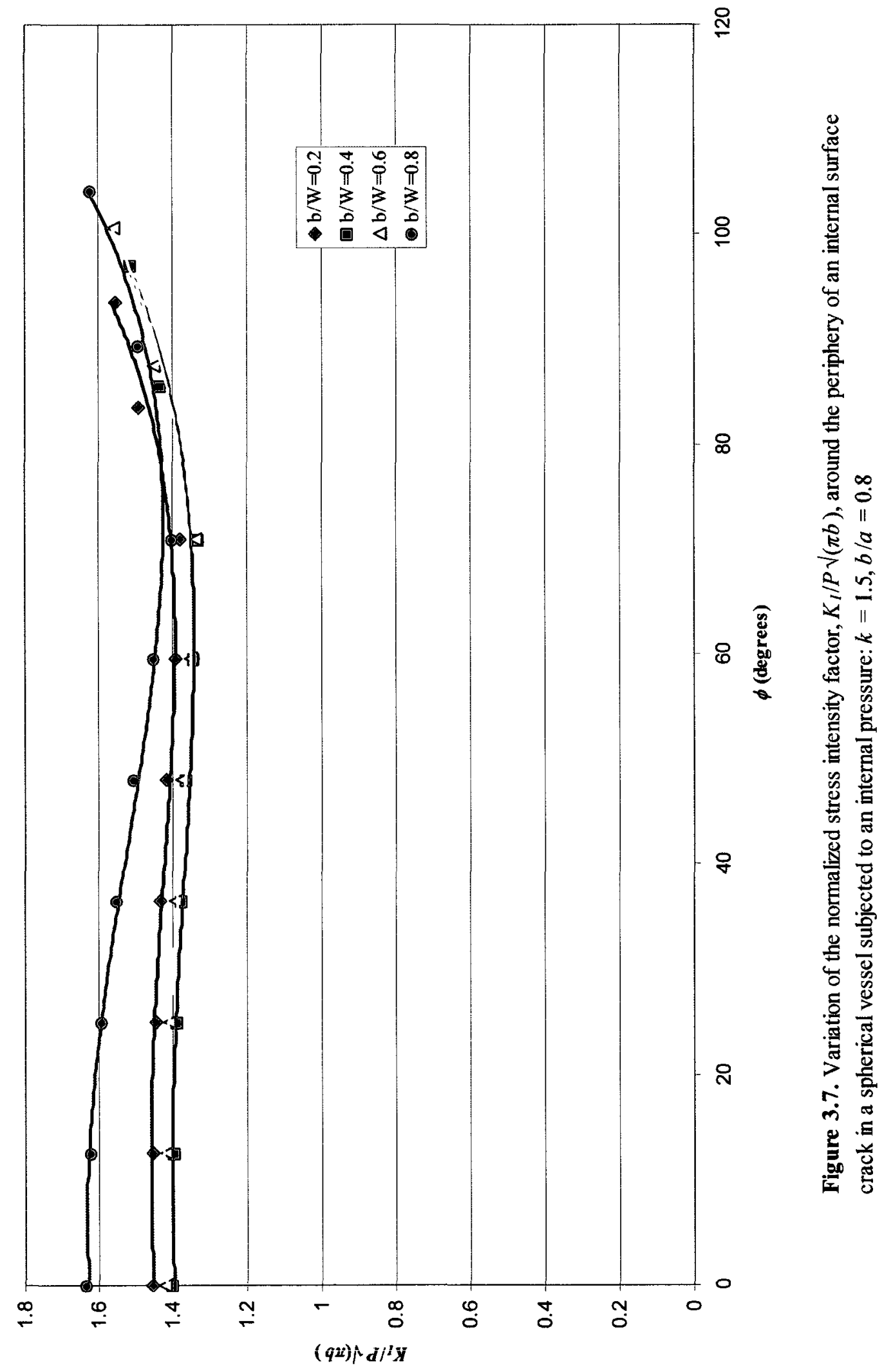




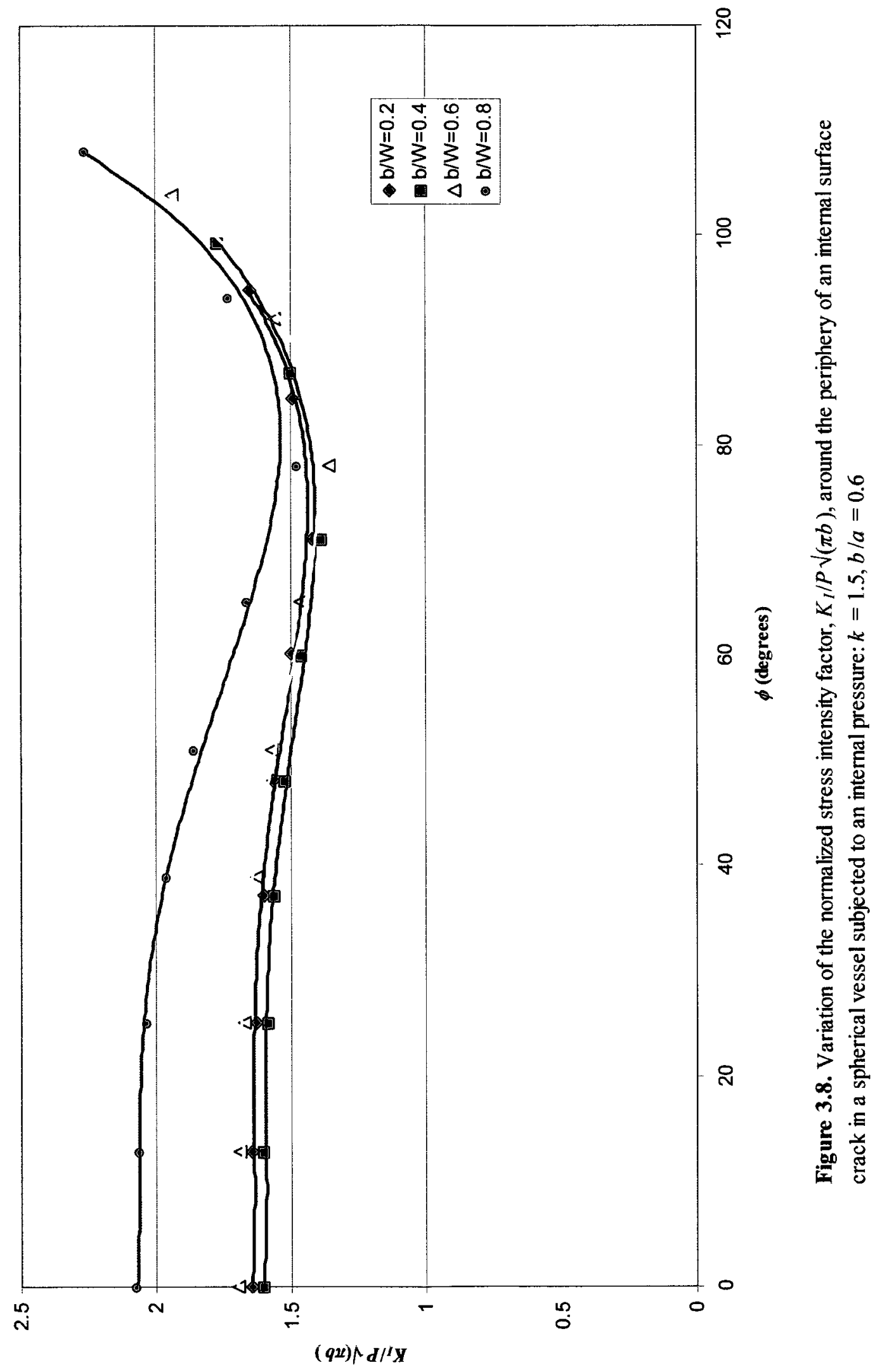




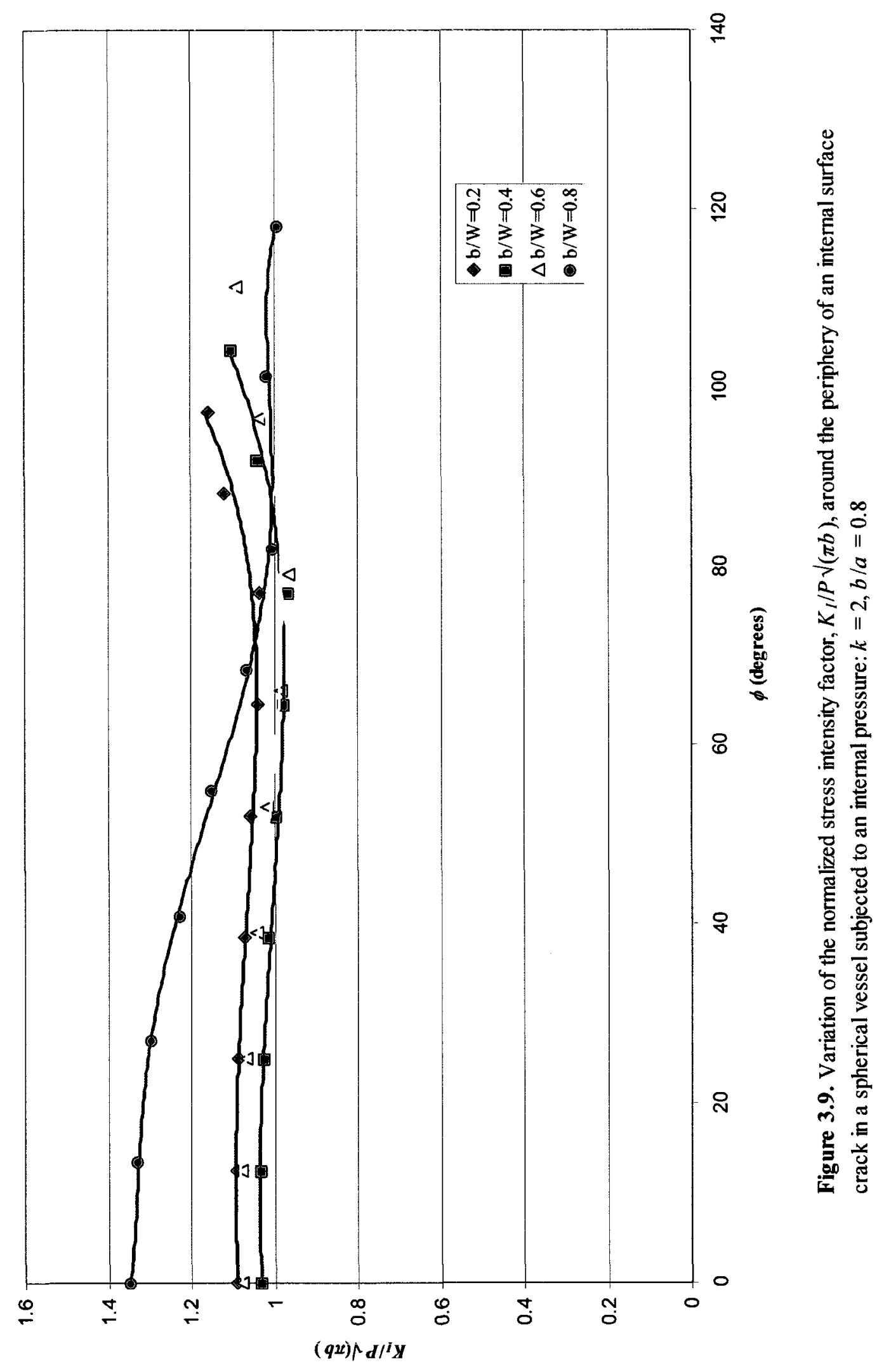




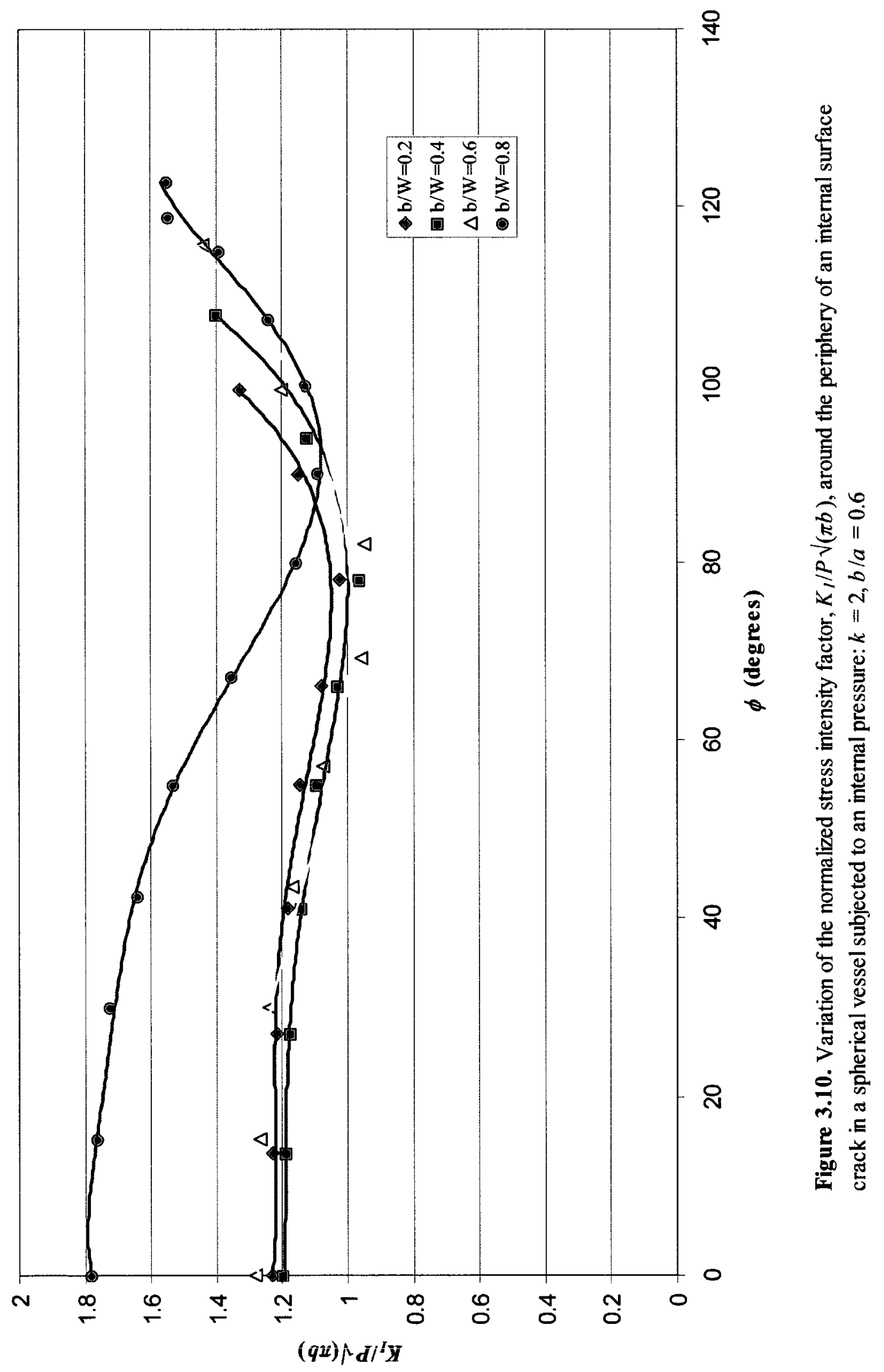




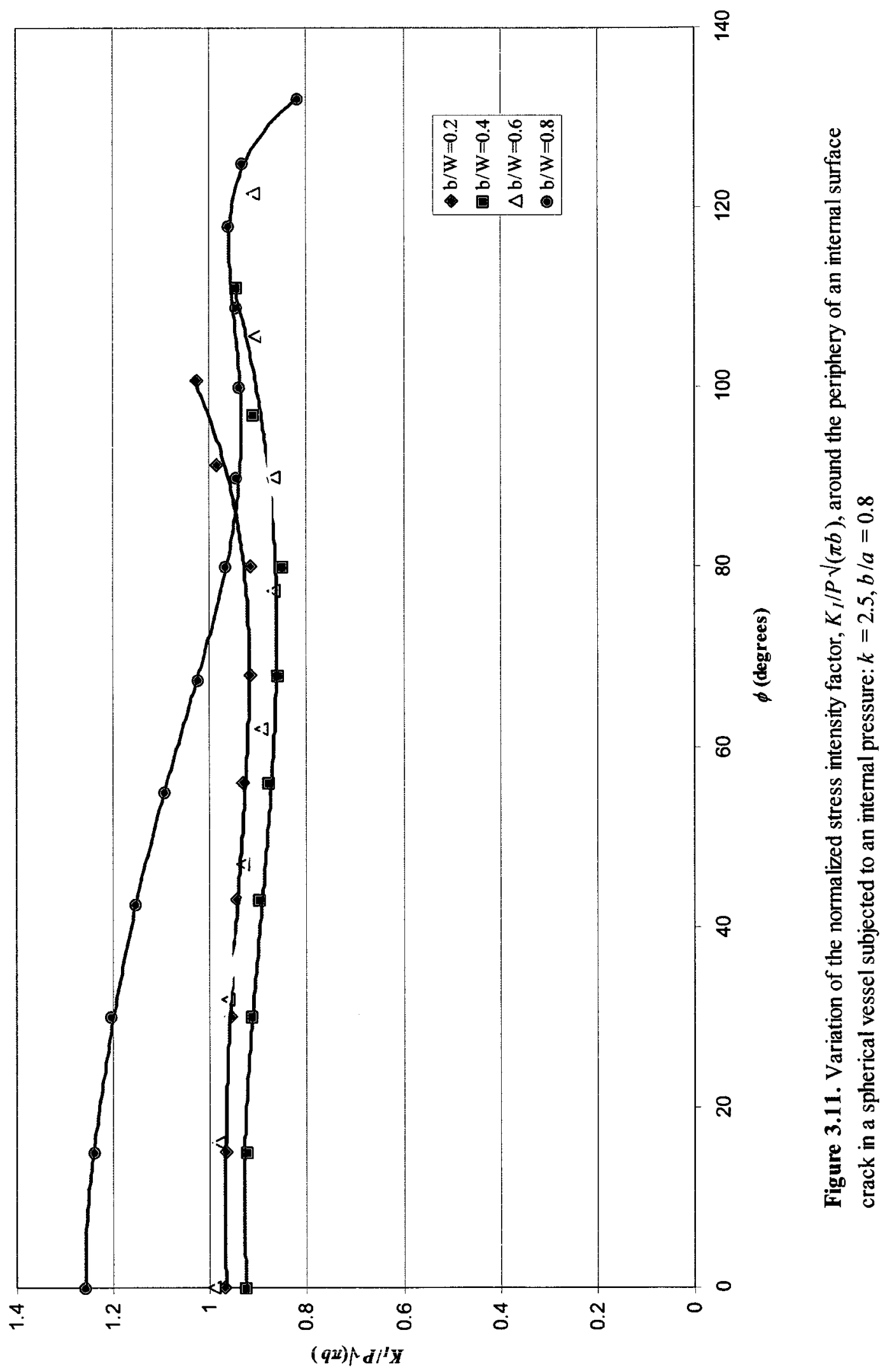




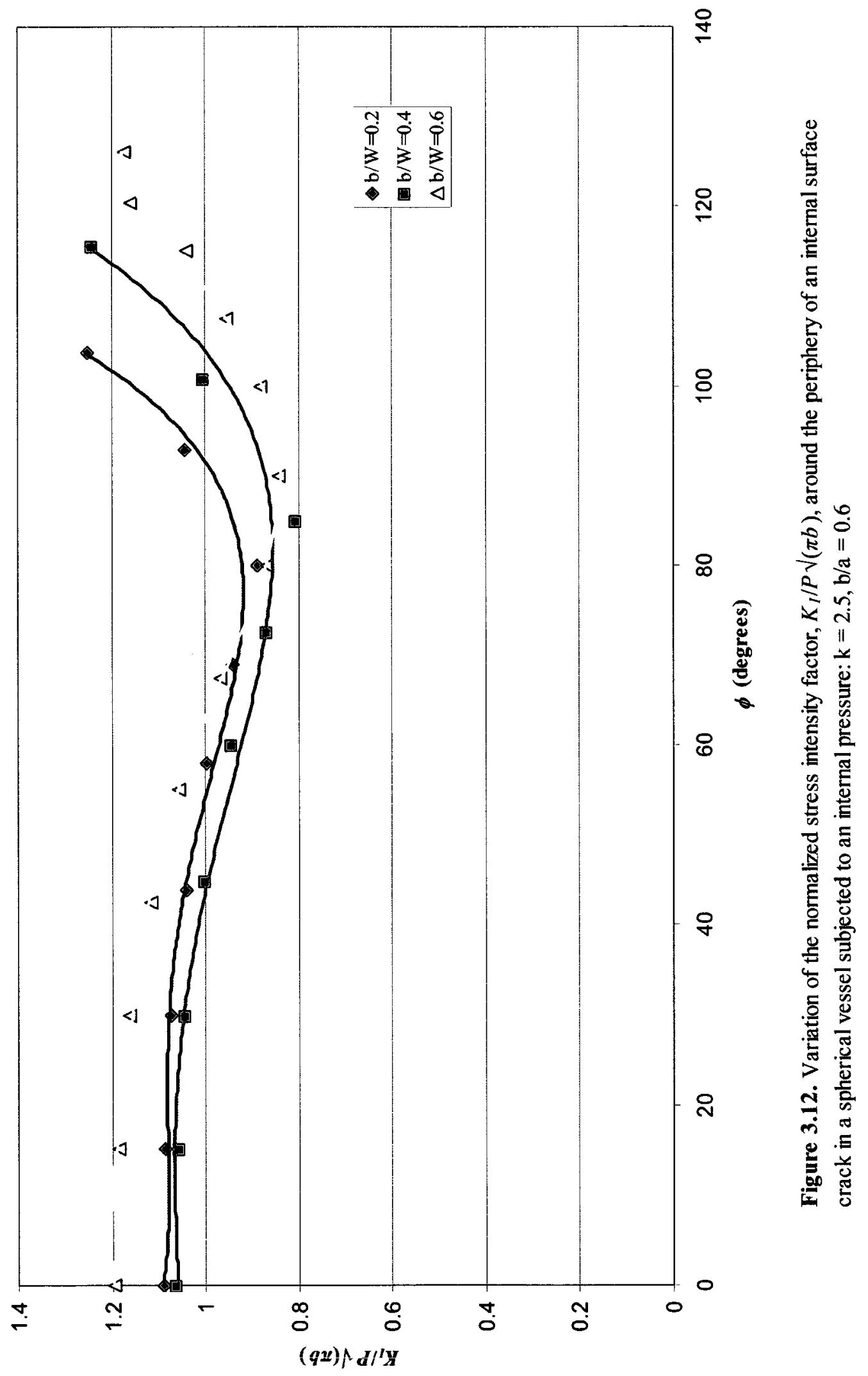




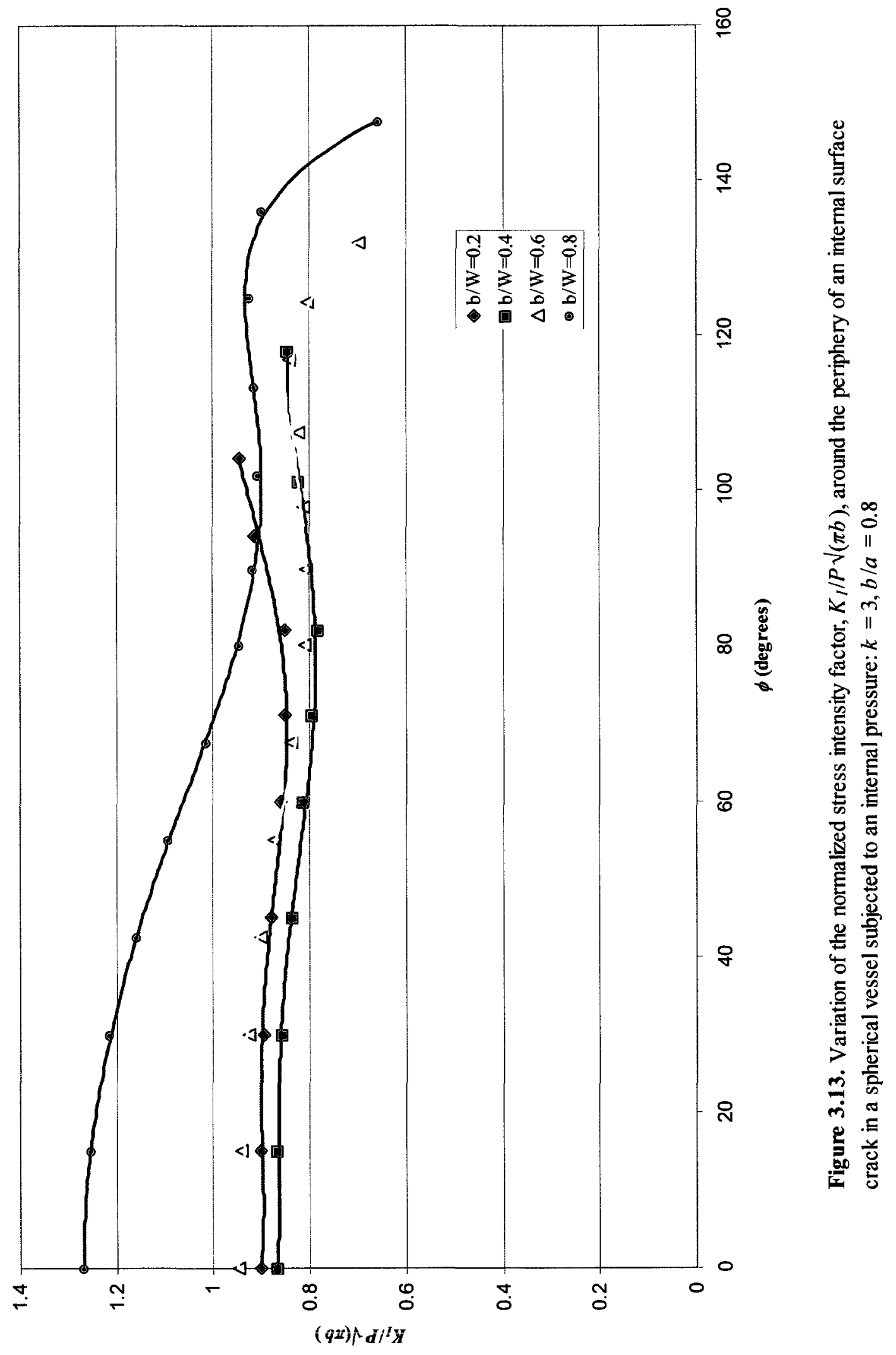




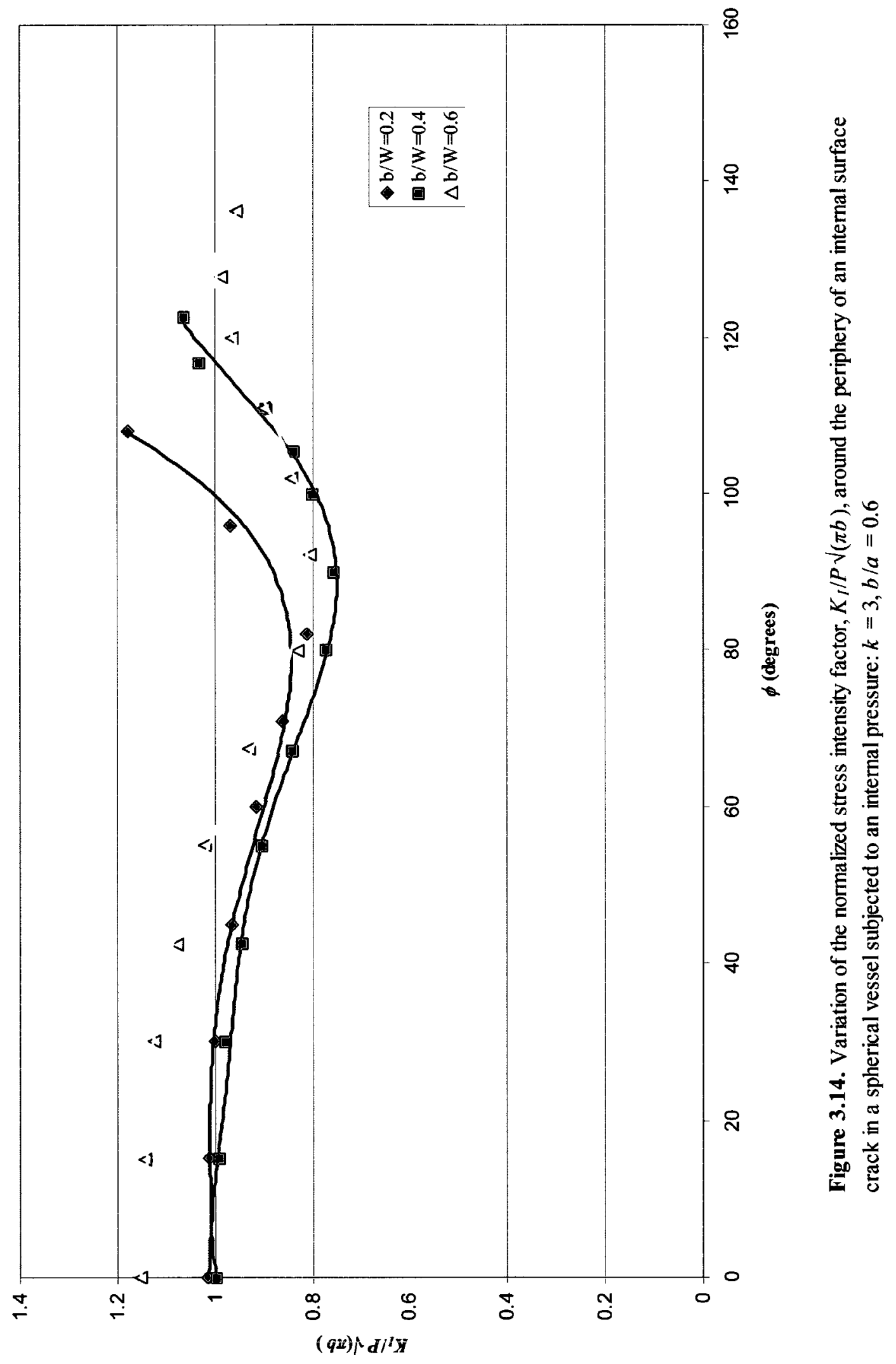




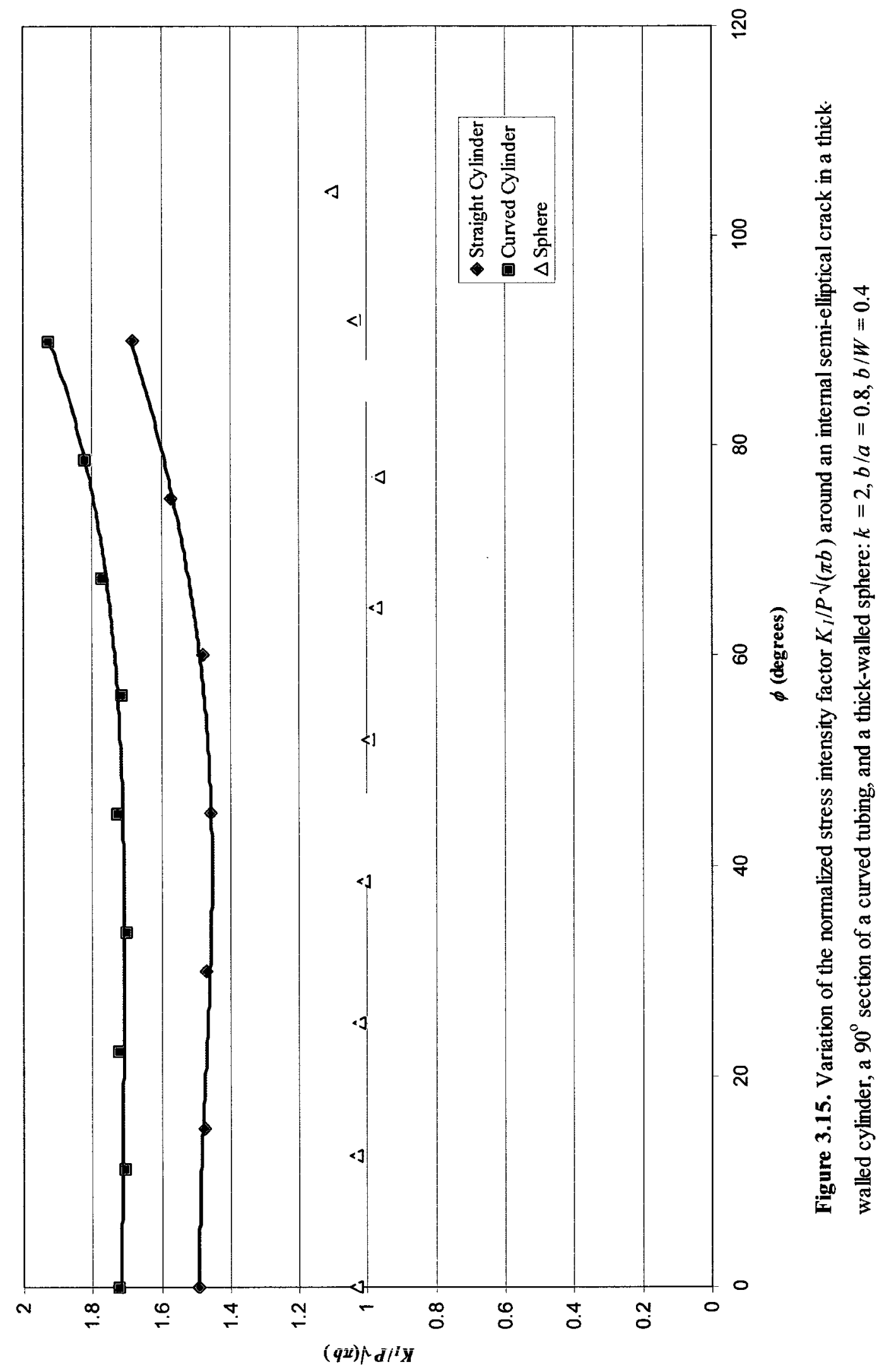




\section{Chapter 4}

\section{Spherical Vessel with a Crossbore}

\subsection{Introduction}

A crossbore is often introduced in a spherical vessel to allow pressurized fluid to be pumped into it. This, however, results in a geometric discontinuity at the intersection of the crossbore and the inner and outer surfaces of the sphere, thereby elevating the circumferential stresses when the vessel is pressurized. Of particular concern is the stress concentration at these locations, since these are typical sites for crack initiation. In this chapter, the problem is defined and a stress analysis is performed on a wide range of geometric combinations to determine the effects of the relative size of the crossbore. A fracture mechanics study is also carried out in which stress intensity factors are determined for a pair of symmetric quarter-circular cracks emanating from the edges of the crossbore at the interior surface of the sphere.

\subsection{Stress Analysis of a Spherical Vessel with a Crossbore}

A diametrical section of a spherical vessel with a crossbore is illustrated in Figure 4.1. To determine the effects of a crossbore on the stresses in the sphere when subjected to an internal pressure, $P$, a parametric study involving the two key radius ratios was carried out. The cases considered were for spheres with radius ratios of

$$
k=R_{2} / R_{l}=1.5,2,2.5,3
$$

and relative crossbore ratios of

$$
R_{s} / R_{l}=0.1,0.125,0.15,0.2,0.25
$$


Poisson's ratio was again taken as 0.3 throughout. Using the axisymmetric BEM, the normalized hoop stress, $\sigma_{\theta} P$, expressed as the circumferential stress factor, $K_{p}$, was determined along the entire axial length of the crossbore for all 20 cases. The hoop stress is of concern because this principal stress has particular influence on crack initiation, growth, and fatigue life (Comlekci et al. (2007)).

\subsubsection{Numerical Model}

Taking advantage of axisymmetry, only the boundary of the radial plane of the problem was modelled, using similar techniques from the numerical example in Section 2.1.3. A typical mesh is shown in Figure 4.2. Around the crossbore, the line elements were decreased in size to more accurately capture the rapidly varying stresses. The number of elements along the axial length of the crossbore varied between 5 and 8 , depending on the thickness of the sphere. The elements were then gradually enlarged to match those away from the crossbore. The numerical model was formed from 23 elements and 47 nodes to 25 elements and 51 nodes. Displacement constraints in the z-direction were applied to the nodes on the $r$ - axis. Here the tractions in the $\mathrm{z}$ - direction are zero, thus these constraints only exist to prevent rigid body motion. A uniform pressure was applied to the internal surface of the sphere and that of the crossbore.

\subsubsection{Results}

The BEM computed circumferential stress factors, $K_{p}$, are listed in Tables 4.1 to 4.4 with respect to the axial position along the crossbore, $z / R_{l}$, from points $\mathrm{A}$ to $\mathrm{B}$ (see Figure 4.1). Since the surfaces of the sphere are curved in the diametrical plane, their intersections with the crossbore occur at different axial positions for each case, which explains the different range of $z / R_{l}$ values between positions $\mathrm{A}$ and $\mathrm{B}$. As an independent check of the solutions, FEM analyses were performed for the set of cases with $k=1.5$. At the inner and outer surface of the crossbore, points $\mathrm{A}$ and $\mathrm{B}$, the discrepancies between the FEM and BEM results were within $2.4 \%$. The typical variation of the circumferential stress factor along the surface of the crossbore, determined using both the BEM and FEM, is 
shown in Figure 4.3 for the $k=1.5, R_{s} / R_{l}=0.1$ case. For this case, 10 quadrilateral elements were required to model the crossbore surface with FEM, while with BEM, it was modelled with just 5 line elements. A mesh refinement study was also performed on $k=1.5, R_{s} / R_{l}=0.1$, to further assess the BEM model. The refinement simply involved adding two more relatively small elements near the stress concentration. Using the more refined mesh, a circumferential stress factor of 3.011 at point $A$ and 2.534 at point $B$ was obtained, a difference of less than $0.25 \%$ from the values obtained using the original mesh. The comparison of the values determined using the BEM and FEM, in addition to the mesh refinement exercise, affirms that the results and the modelling strategies are adequate.

The variation of the circumferential stress factor from point $A$ to point $B$ for all the cases are presented in Figures 4.4 to 4.7. The results for the smaller crossbores show that the maximum circumferential stress factor is at point $A$, the intersection of the interior surface of the sphere and the crossbore. Along the edge $A B$, as the exterior surface of the sphere is approached, the circumferential stress factor increases, unlike a similarly sized radial circular crossbore in a thick-walled straight cylinder (Tan and Fenner (1980), and Makulsawatudom et al. (2004)), which shows no such rise in hoop stress. However, if the size of the radial crossbore in the cylinder is relatively small $\left(R_{s} / R_{1}<0.05\right)$, then a rise in hoop stress near the exterior surface can be seen, as demonstrated by the findings of Comlekci et al. (2007). Similar trends to those in the present work can also occur in a cylinder if the crossbore is offset, or of the elliptical form (Masu (1997 and 1998)). Also unforeseen, the variations of the circumferential stress factor for the larger crossbores show that the maximum value in the crossbore can be located at its intersection with the exterior surface. However, similar observations can be made in a cylinder as well. If the crossbore is of the elliptical form, and the normalized hoop stress is determined along its major axis, then the variation resembles that of $k=1.5, R_{s} / R_{I}=0.25$ from the present work. The hoop stress increases from the interior surface to its maximum value located at the exterior surface (Masu (1997)). Although the unexpected normalized hoop stress variations in a spherical vessel with a crossbore can also be seen in some cylindrical vessels, the values of this quantity at a crossbore in a cylinder are much higher than those of a sphere with the same radius and crossbore ratios. This is shown in Figure 4.8, where 
the results of $k=2, R_{S} / R_{l}=0.1$ from the present study are compared to the work done by Comlekci et al. (2007) on thick-walled cylinders.

\subsection{Quarter - Circular Cracks at a Crossbore in a Spherical Vessel}

As demonstrated in the previous section, the maximum hoop stress in a spherical vessel with a crossbore is located at its intersection with either the interior or exterior surface of the sphere. Therefore, these locations would most likely be the sites for crack initiation. To investigate the problem of cracks existing at the crossbore in a spherical vessel, normalized stress intensity factors were obtained for a sample of the geometric possibilities. For this study, as illustrated in Figure 4.9, two cracks were taken to be symmetrical and part circular; they are almost quarter-circular, and for convenience will be referred to as such. The radius ratio considered was $k=2$, with crossbore radiuses of

$$
R_{s} / R_{l}=0.1,0.15,0.2,0.25
$$

and relative crack depths of

$$
b / W=0.2,0.4,0.6,0.8
$$

where $W$ is the thickness of the sphere. The internal pressure, $P$, was assumed to also act on the crossbore surface, and crack faces.

\subsubsection{Numerical Model}

As shown in Figure 4.9, the crossbore was assumed to contain two cracks in the same plane, which added a plane of symmetry. Therefore, only a one-quarter model was required, as shown by the mesh for $R_{s} / R_{l}=0.2, b / W=0.4$, in Figure 4.10 . The size of the numerical model varied from 142 elements and 398 nodes to 157 elements and 447 
nodes. For consistently accurate results, between four and six elements were placed along the crack front. The length, $l$, of the elements perpendicular to the crack front was set to $0.15 a$, and then gradually enlarged to match the mesh distribution away from the crack and the crossbore. Displacement was constrained on all the planes of symmetry in their normal directions, and the nodes on the $\mathrm{x}_{1}-\mathrm{x}_{2}$ plane were restrained in the $\mathrm{x}_{3}$ - direction to preclude rigid body motion. A uniform pressure was applied to the internal surface of the sphere, the surface of the crossbore, and the crack face.

\subsubsection{Results}

The normalized stress intensity factors for quarter-circular cracks at a crossbore in a spherical vessel are presented in Tables 4.5 to 4.8 in relation to $\phi$, the position on the crack front measured from the crossbore. They were determined using both the traction formula and displacement formulas. It is evident from the normalized stress intensity factor tables that deviations of generally less than $3 \%$ occur between the results from both formulas, except for the values corresponding to the free surfaces. For some of the relatively large cracks, the adjacent mid-side node and corner node were also affected. Similar to the experience described in Chapter 3, it was found that adding one or two more elements along the crack front close to the area of concern reduced the discrepancies at the mid-side node. However it was observed that if the elements were relatively too small, the accuracy of the following corner node on this crack element suffered. By carefully refining the mesh and avoiding an extreme element size, the normalized stress intensity factors from the traction formula and displacement formulas at both these nodes generally varied by less than $6 \%$.

Figures 4.11 to 4.14 show the normalized stress intensity factor variation along the periphery of the cracks determined using the displacement formulas. For all the cases, the maximum value occurs at the intersection of the crack with the crossbore, consistent with the location of the stress concentration. Moving along the crack fronts from the crossbore, the normalized stress intensity factor decreases and then stays relatively constant until the intersection with the internal surface of the sphere. Here, at some distance away from the crossbore, the trends observed in Chapter 3 are evident. The normalized stress intensity 
factor tends to remain fairly uniform, and for the larger crack sizes, it actually decreases near this free surface. This is similar to the cracked sphere without a crossbore, suggesting that the stress concentration is relatively localized. Tan and Fenner (1980) collected similar results during their study of thick-walled cylinders. They found that the normalized stress intensity factor is generally at its maximum value at the intersection of the crack fronts and the crossbore. At some distance away, the variation of this quantity then resembles that of a cracked cylinder without a crossbore.

\subsection{Conclusions}

Stress analyses were performed on a number of geometric combinations of a spherical vessel with a crossbore. It was determined that the maximum value of normalized hoop stress typically occurred at the intersection of the crossbore and the internal surface of the sphere. However, the results of the present study showed that the maximum normalized hoop stress in a relatively larger crossbore could move to the exterior surface, similar to elliptical and offset crossbores in thick-walled cylinders. Stress concentrations such as crossbores are typical locations for crack initiations. A selection of possible crossbore geometries containing two symmetric quarter-circular cracks in the same plane was analyzed. It was determined that the maximum normalized stress intensity factor occurred where the crack front intersected with the crossbore. Since the effects of the stress concentration are localized, the trends away from the crossbore resembled those from Chapter 3 . 


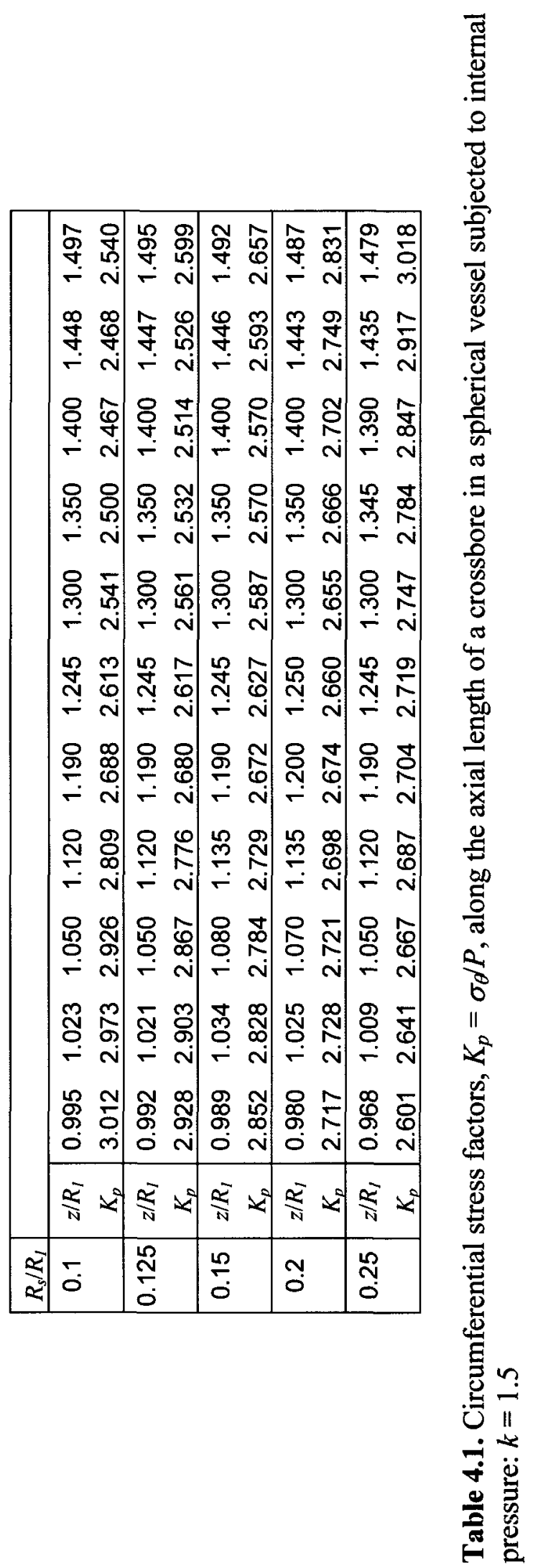




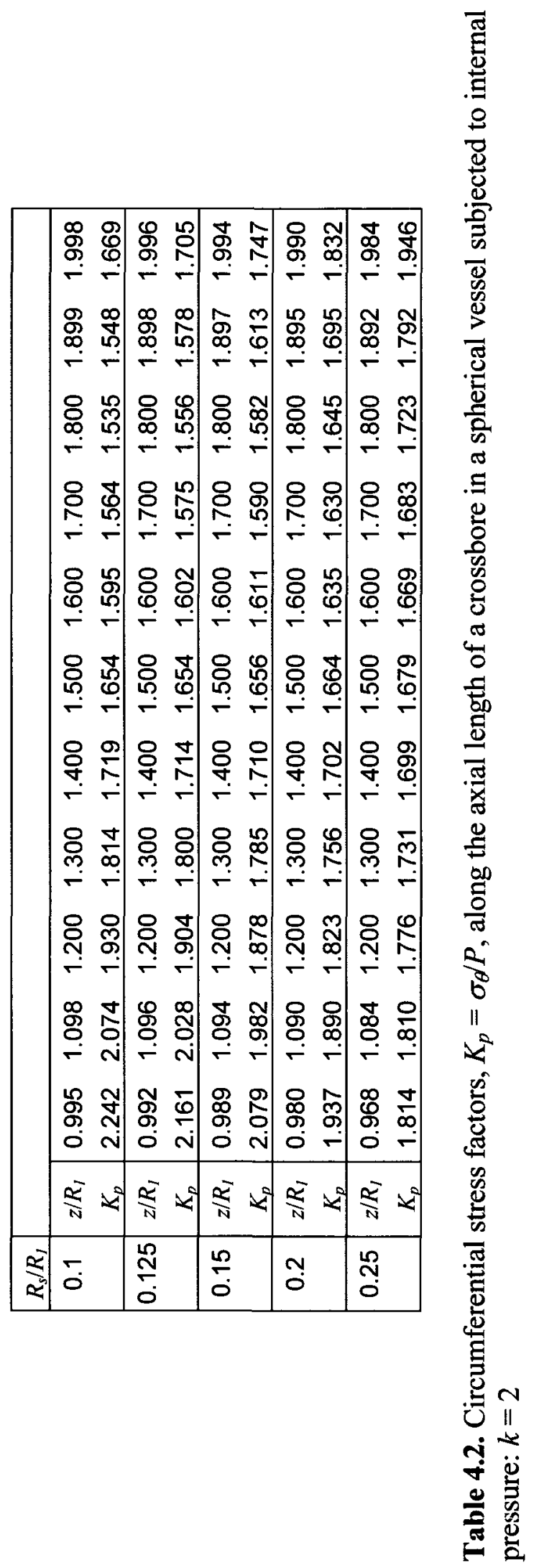


惌

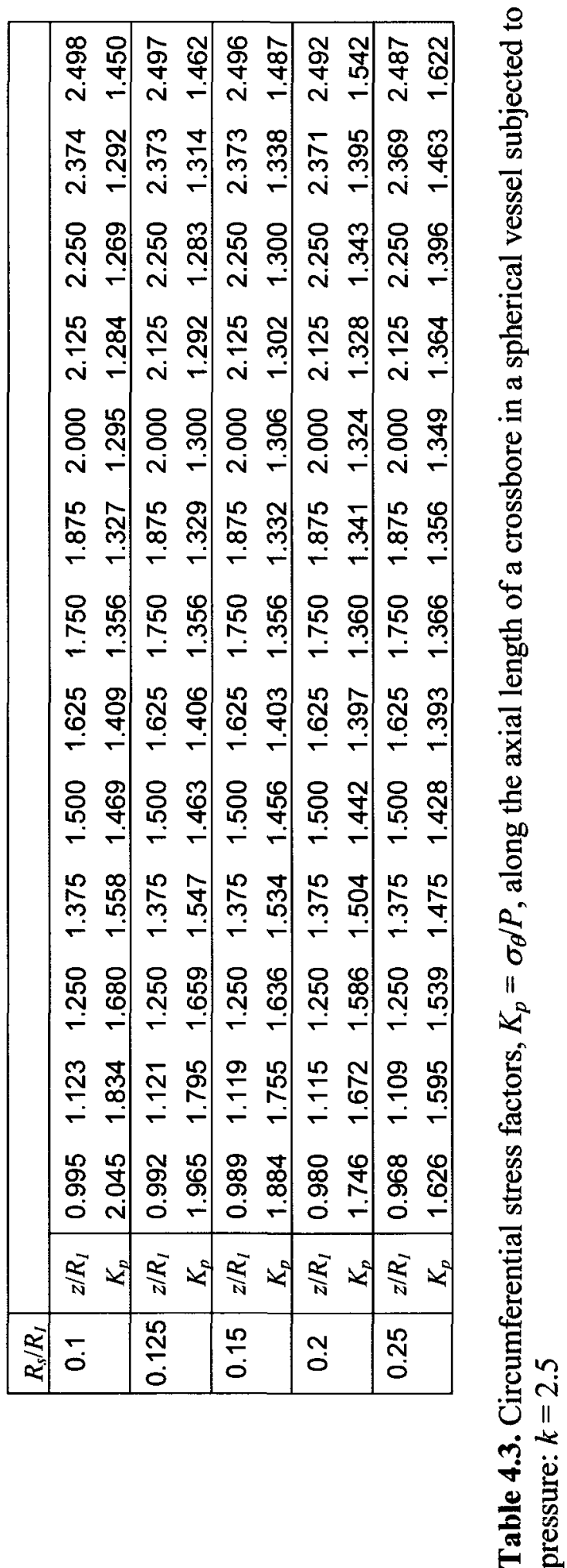




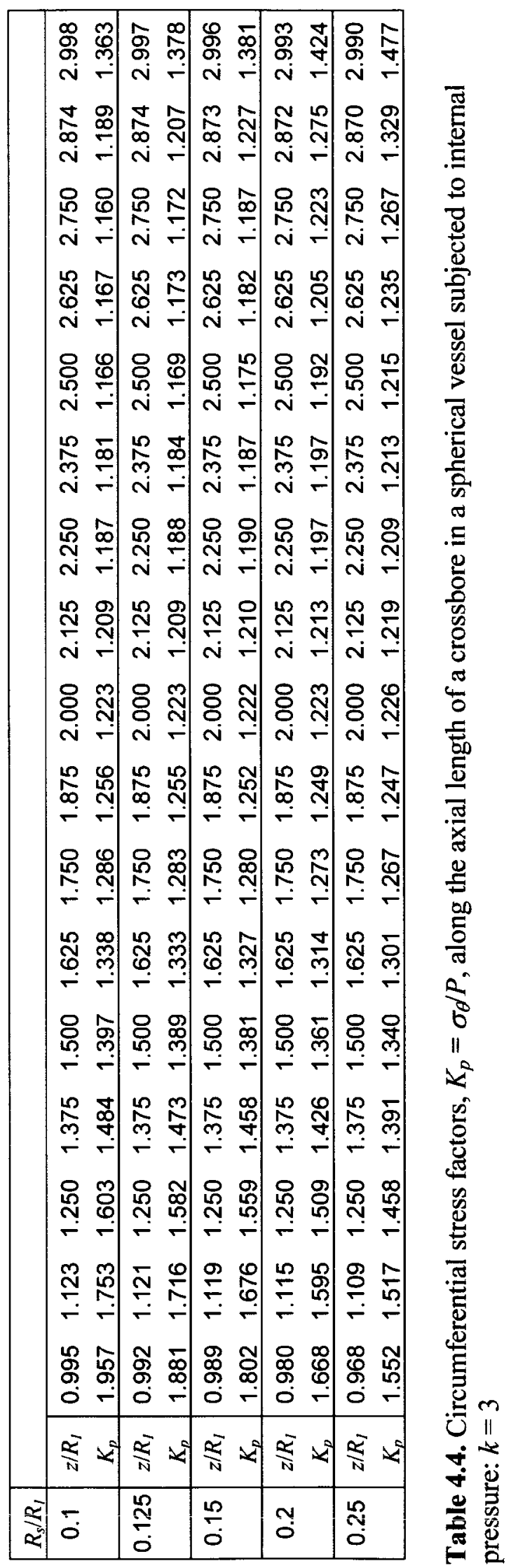




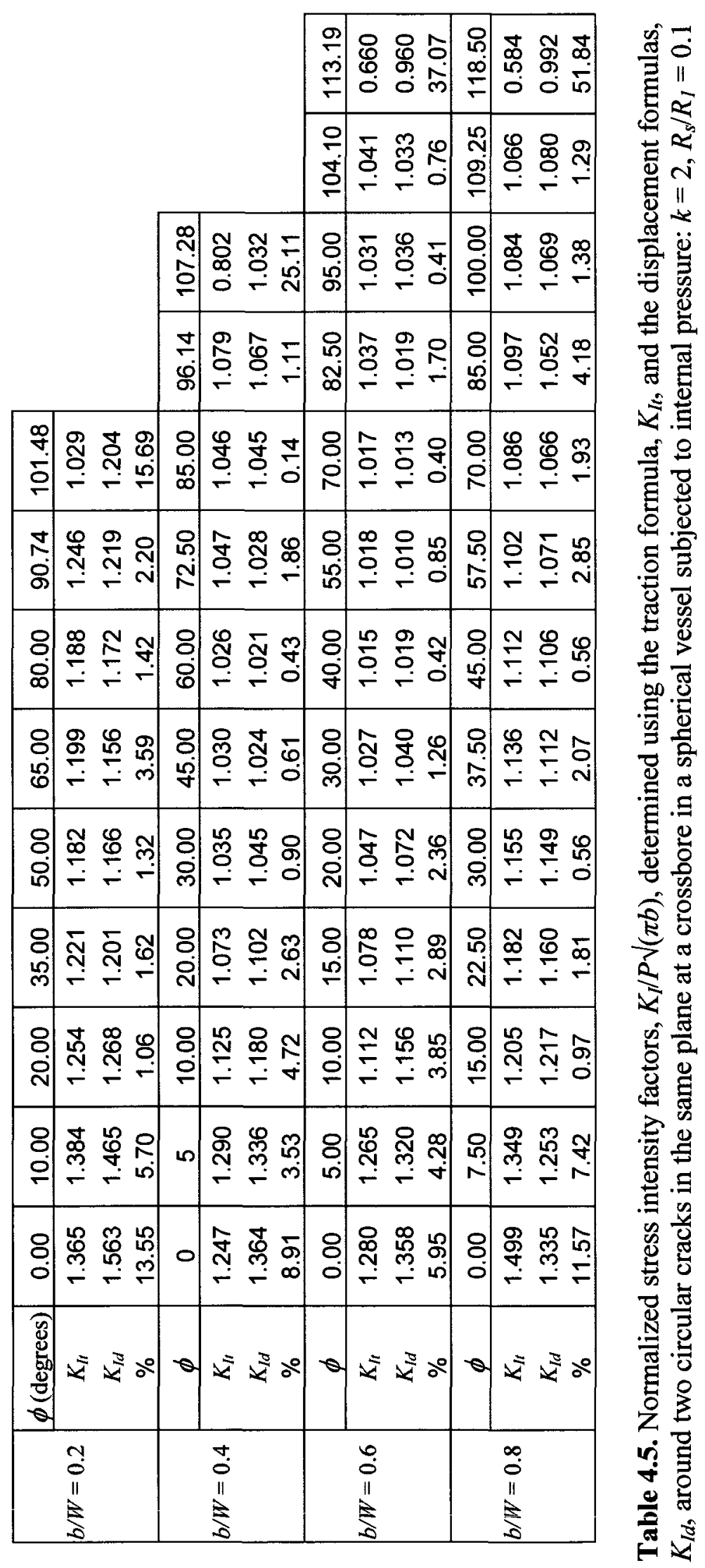




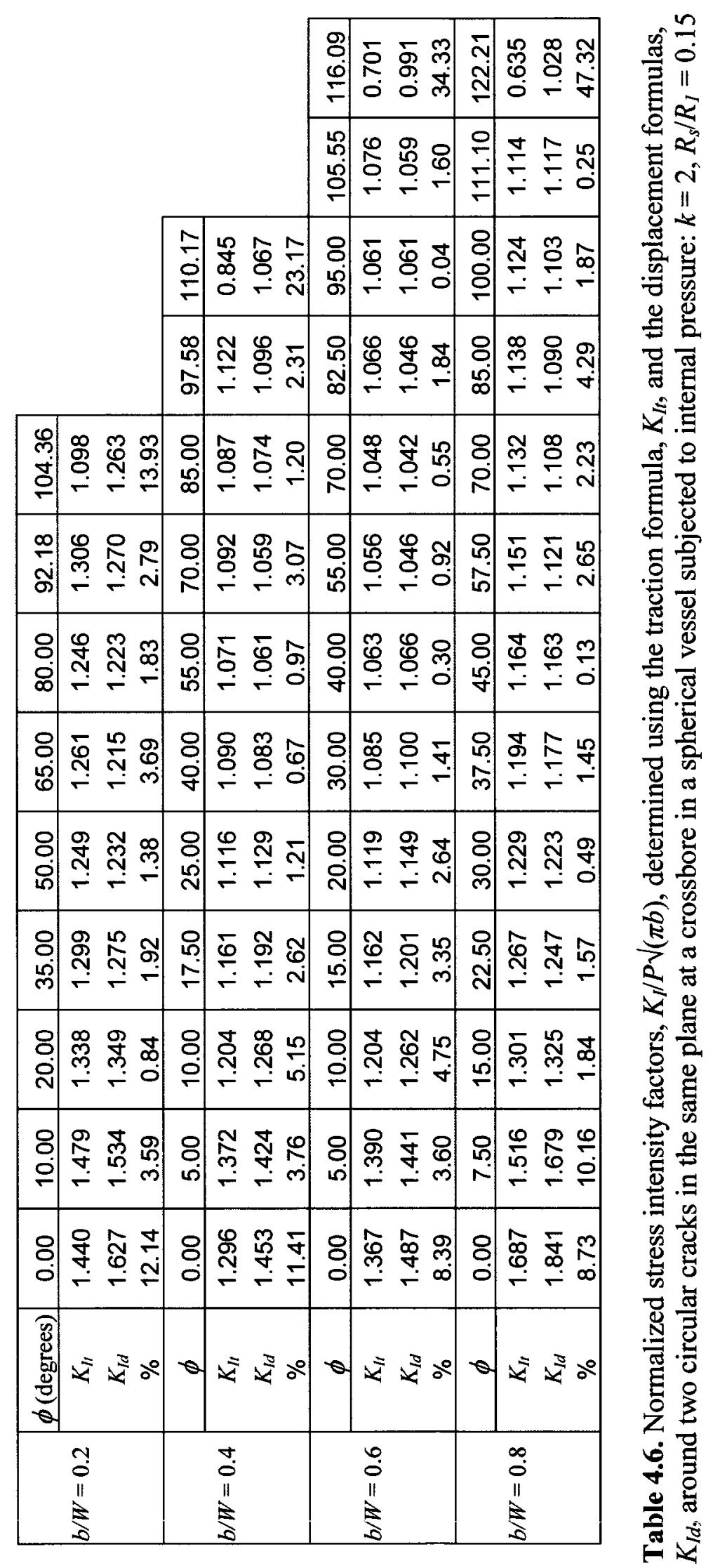




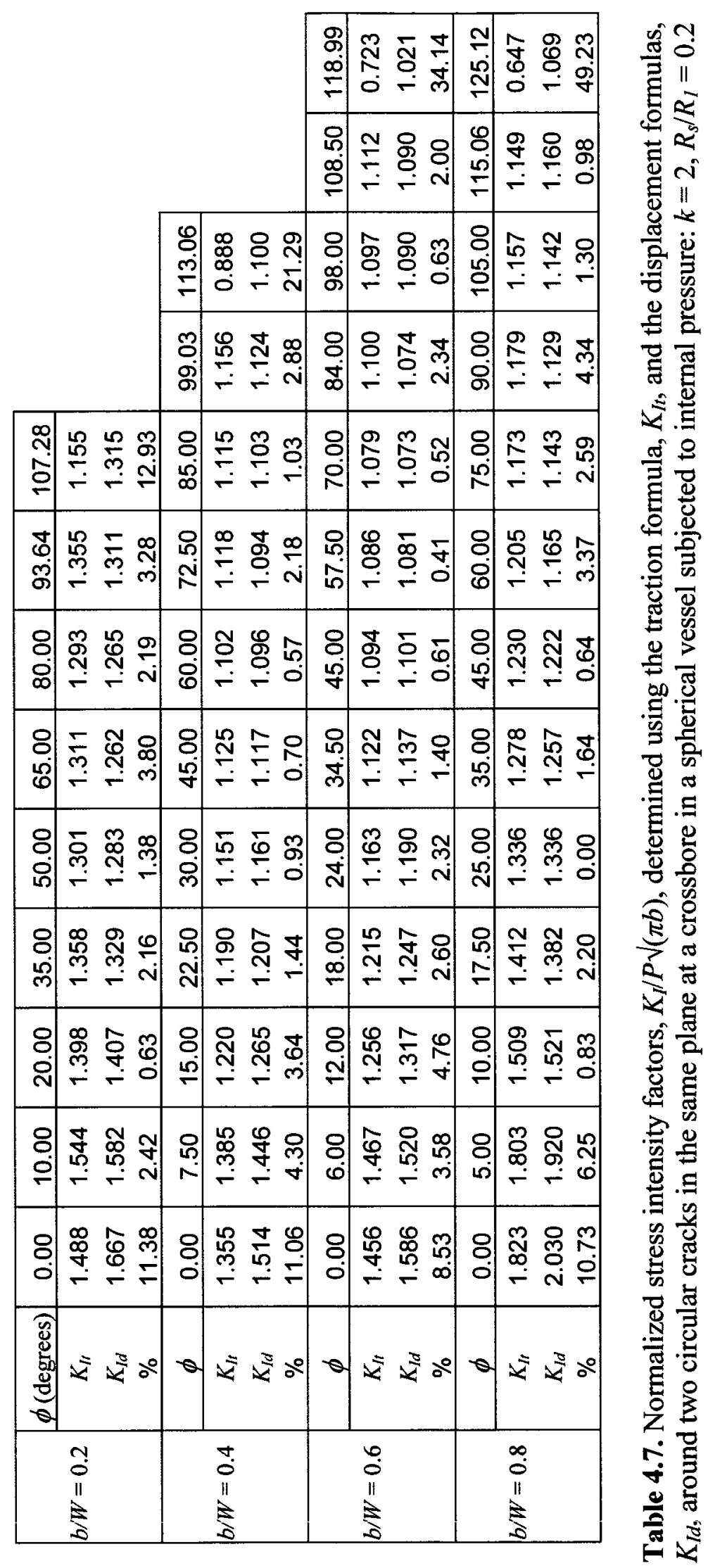




\begin{tabular}{|c|c|c|c|c|c|c|c|c|}
\hline & \multirow[b]{3}{*}{$\begin{array}{l}\sigma \\
\dot{\sigma} \\
\sigma \\
\sigma\end{array}$} & \multirow[b]{3}{*}{ 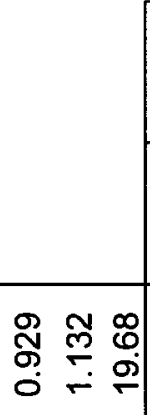 } & $\begin{array}{l}\stackrel{ \pm}{\sigma} \\
\stackrel{\sim}{\leftarrow}\end{array}$ & \multirow{2}{*}{ 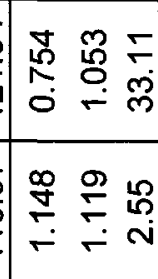 } & \multirow{2}{*}{ 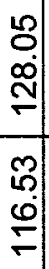 } & 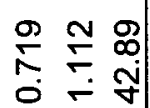 & \multirow{4}{*}{ 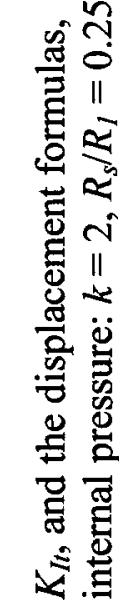 } \\
\hline & & & & $\begin{array}{l}\text { aे } \\
\text { 음 }\end{array}$ & & & 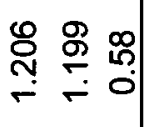 & \\
\hline & & & & 염 & $\stackrel{\bar{m}}{\stackrel{\infty}{\leftarrow}} \stackrel{\infty}{\leftarrow} \stackrel{m}{\leftarrow}$ & $\begin{array}{l}8 \\
\text { : } \\
\text { 인 }\end{array}$ & 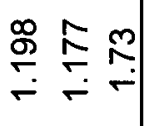 & \\
\hline & & $\begin{array}{l}0 \\
0 \\
8 \\
8\end{array}$ & 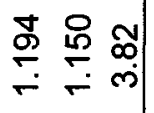 & $\begin{array}{l}8 \\
8 \\
\infty \\
\infty\end{array}$ & 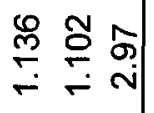 & $\begin{array}{l}8 \\
8 \\
8\end{array}$ & 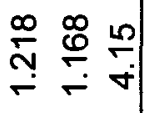 & \\
\hline 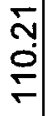 & 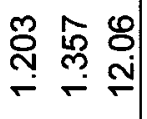 & $\begin{array}{c}8 \\
1 \\
\infty\end{array}$ & 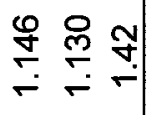 & $\begin{array}{l}8 \\
0 \\
0\end{array}$ & 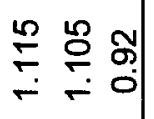 & $\begin{array}{l}8 \\
0 \\
10\end{array}$ & 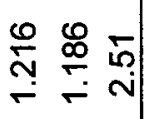 & 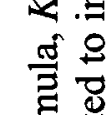 \\
\hline Е & 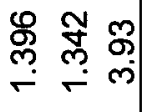 & $\begin{array}{l}\text { 오 } \\
\text { Ni }\end{array}$ & 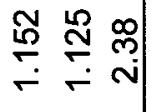 & 员 & 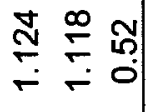 & $\begin{array}{l}8 \\
8 \\
8\end{array}$ & 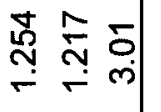 & 0 \\
\hline $\begin{array}{l}8 \\
8 \\
\varnothing\end{array}$ & 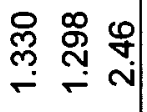 & $\begin{array}{l}8 \\
8 \\
0\end{array}$ & $\begin{array}{ccc}\mathscr{m} & \bar{m} & 0 \\
\check{\sigma} & \check{\sigma} & 0\end{array}$ & $\begin{array}{l}8 \\
8 \\
\text { เి }\end{array}$ & 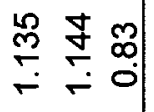 & $\begin{array}{l}8 \\
\text { ஸे }\end{array}$ & 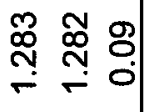 & 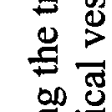 \\
\hline $\begin{array}{l}8 \\
10 \\
0\end{array}$ & 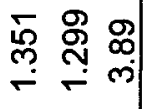 & $\begin{array}{c}8 \\
8+1 \\
4\end{array}$ & 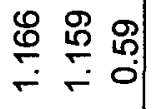 & 員 & 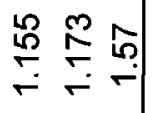 & $\begin{array}{c}8 \\
\text { 1. }\end{array}$ & 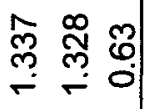 & \\
\hline $\begin{array}{l}8 \\
8 \\
8\end{array}$ & 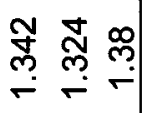 & $\begin{array}{l}8 \\
\text { ¿ } \\
\text { | }\end{array}$ & 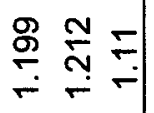 & 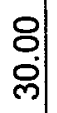 & 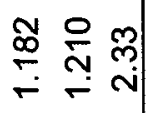 & $\begin{array}{c}8 \\
\text { ஸे }\end{array}$ & 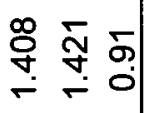 & $\frac{8}{8}$ \\
\hline 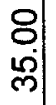 & 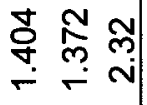 & $\begin{array}{l}\text { ㅇ. } \\
\text { N̦} \\
\text { N }\end{array}$ & 寺 & $\begin{array}{l}\text { : } \\
\text { N̦}\end{array}$ & 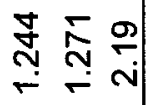 & 융 & 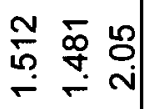 & \\
\hline $\begin{array}{l}\text { ㅇ. } \\
\text { ㅇ. }\end{array}$ & 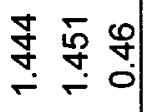 & $\begin{array}{l}8 \\
10 \\
10\end{array}$ & 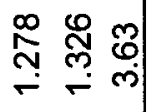 & $\begin{array}{l}8 \\
\stackrel{8}{\circ} \\
\stackrel{\omega}{\circ}\end{array}$ & $\begin{array}{lll}\stackrel{g}{g} & \infty & m \\
\stackrel{N}{*} & \stackrel{m}{*} & m\end{array}$ & 임 & 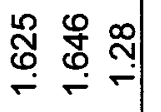 & $\pi$ \\
\hline $\begin{array}{l}\text { 임 } \\
\circ\end{array}$ & Љু & 옹 & 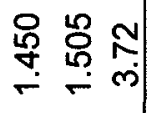 & 옥 & 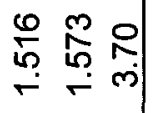 & $\begin{array}{l}8 \\
10\end{array}$ & 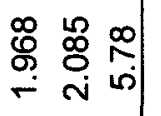 & \\
\hline ㅇ. & 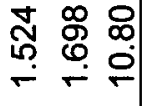 & $\begin{array}{l}8 \\
\dot{0}\end{array}$ & 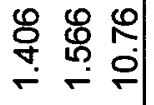 & $\begin{array}{l}8 \\
0\end{array}$ & 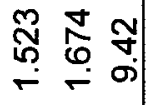 & $\begin{array}{l}8 \\
8\end{array}$ & 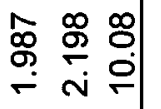 & 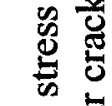 \\
\hline $\begin{array}{l}0 \\
0 \\
0 \\
0 \\
0 \\
0 \\
0\end{array}$ & $\bar{x} \quad x$ & 이 & 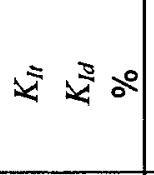 & $\theta$ & $\pm x^{3}$ & $\theta$ & $= \pm$ & 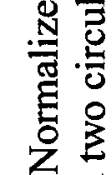 \\
\hline & $\begin{array}{c}\text { a } \\
0 \\
11 \\
3\end{array}$ & & $\begin{array}{l}5 \\
0 \\
11 \\
5 \\
0\end{array}$ & & $\begin{array}{l}0 \\
0 \\
11 \\
0 \\
0\end{array}$ & & $\begin{array}{l}\infty \\
0 \\
0 \\
0\end{array}$ & 3 \\
\hline
\end{tabular}




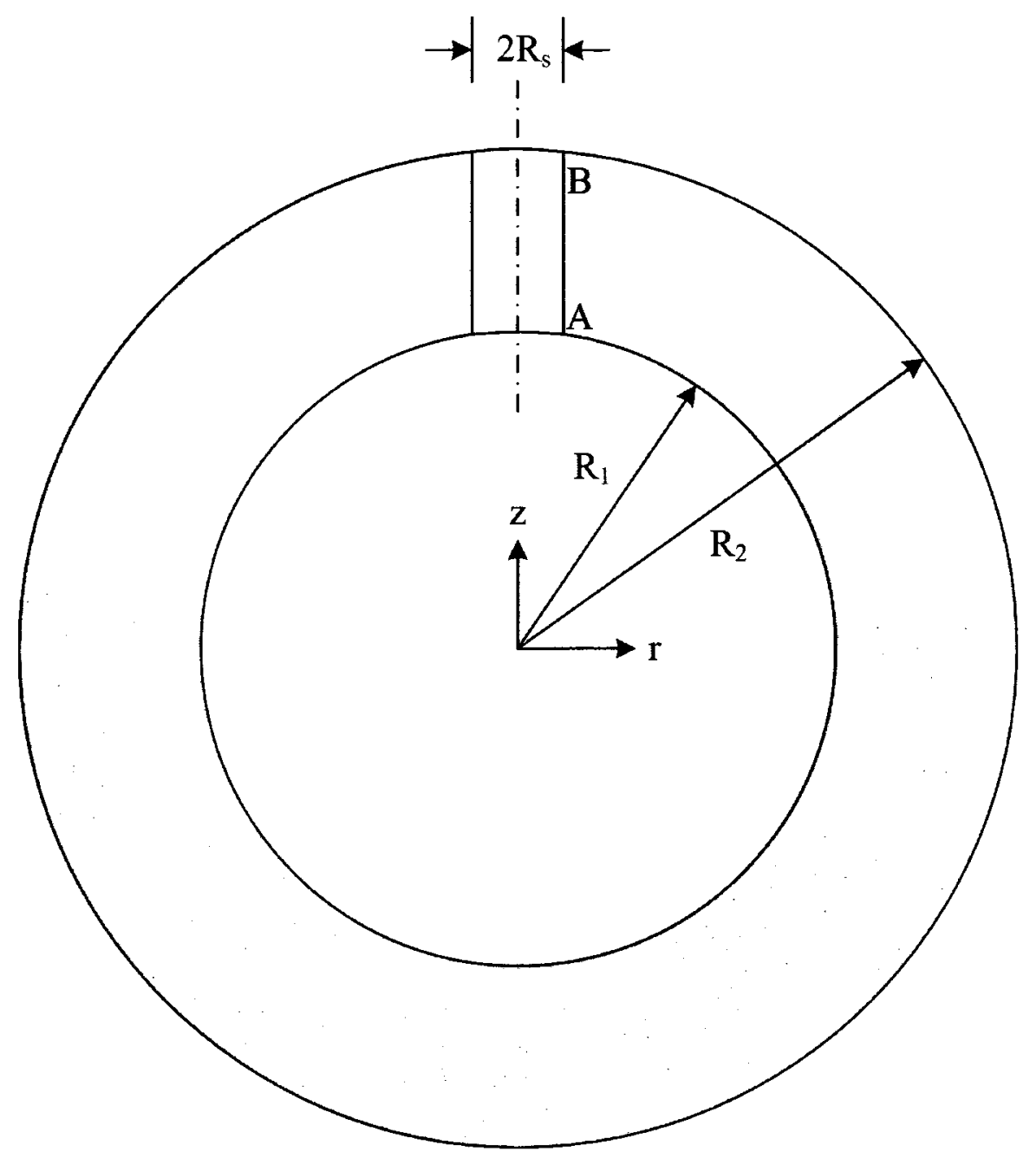

Figure 4.1. Diametrical cross-section of a spherical vessel with a crossbore 


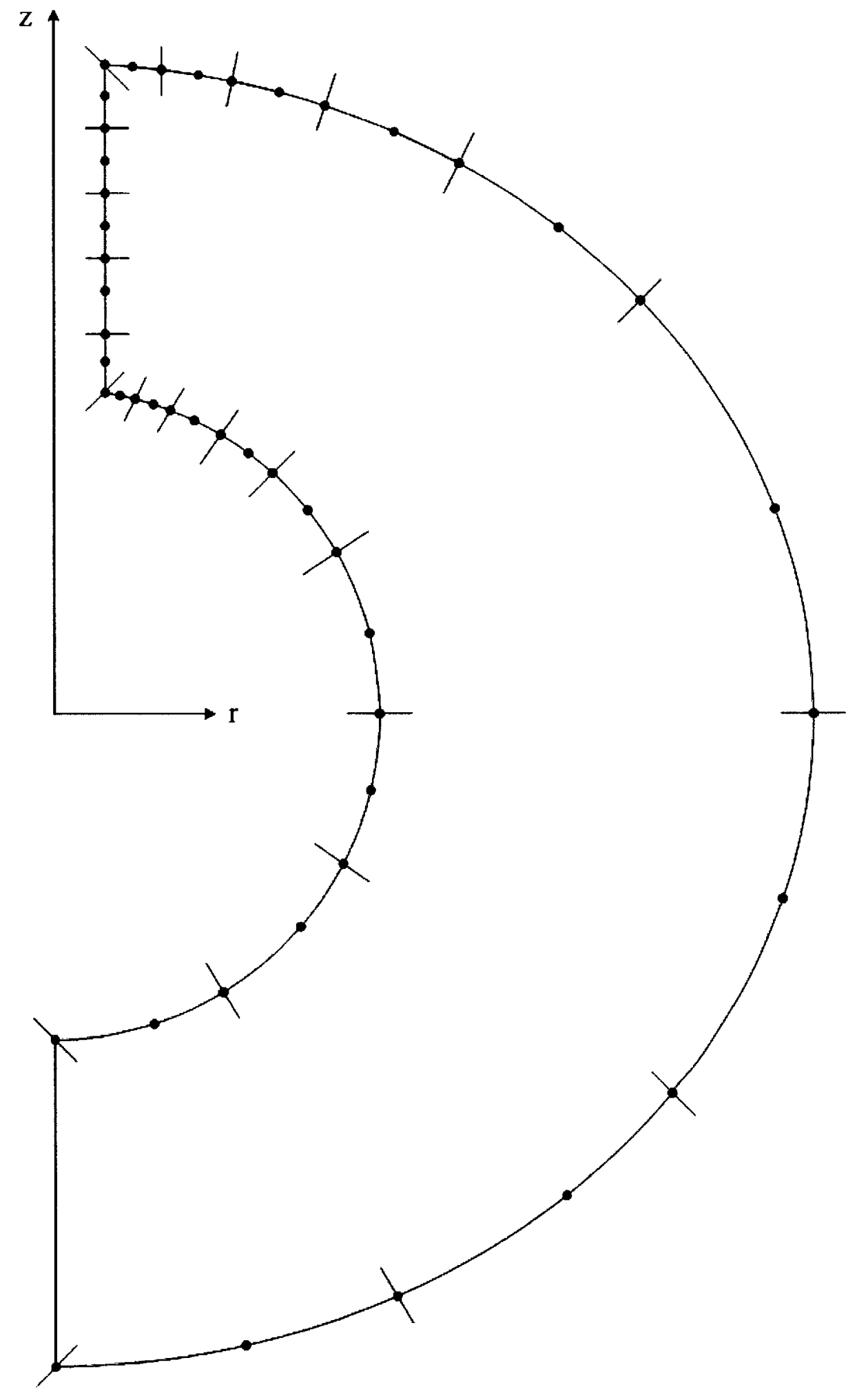

Figure 4.2. Axisymmetric BEM mesh of a spherical vessel with a crossbore: $k=2$, $R_{S} / R_{I}=0.15$ 


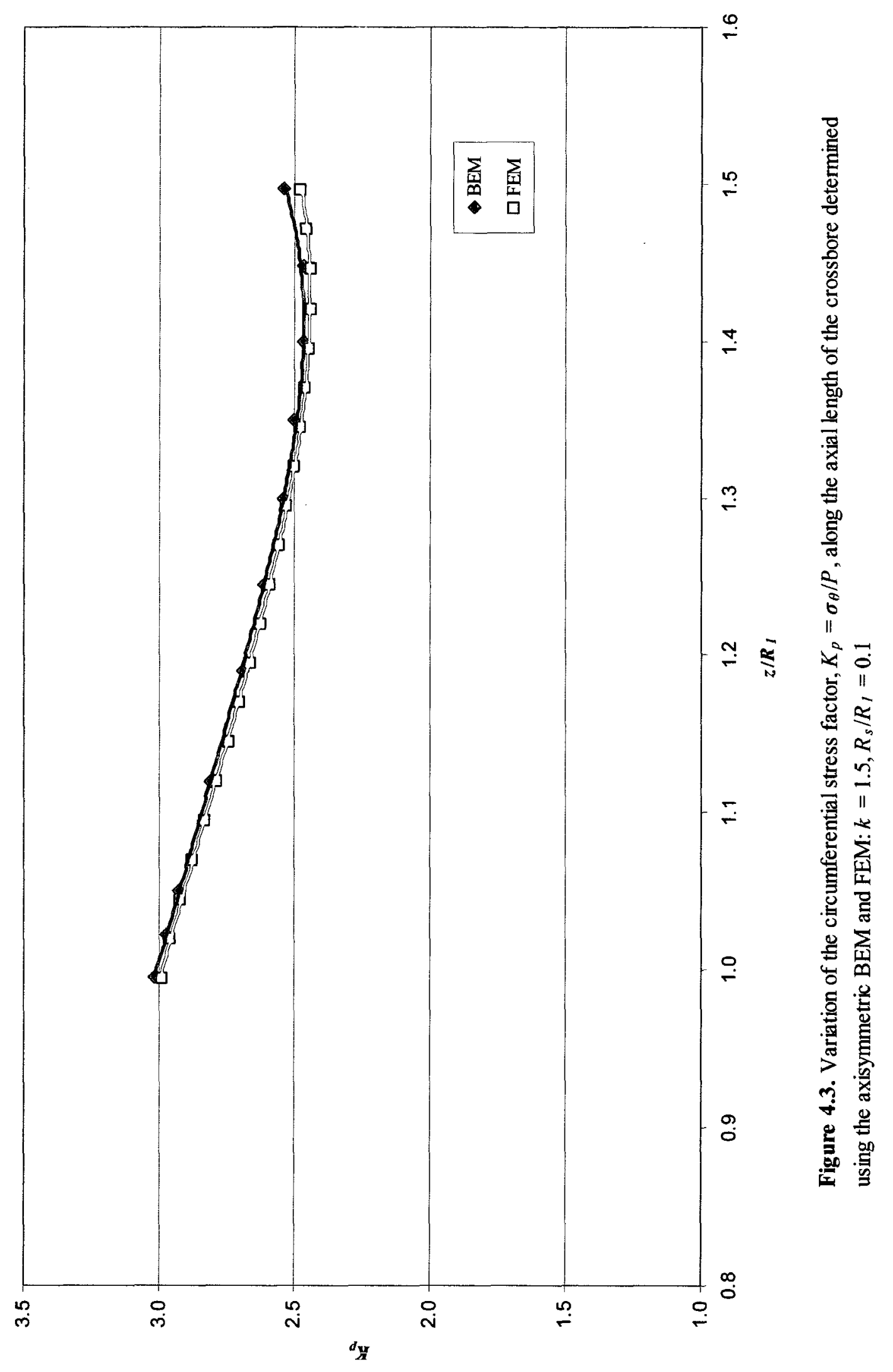




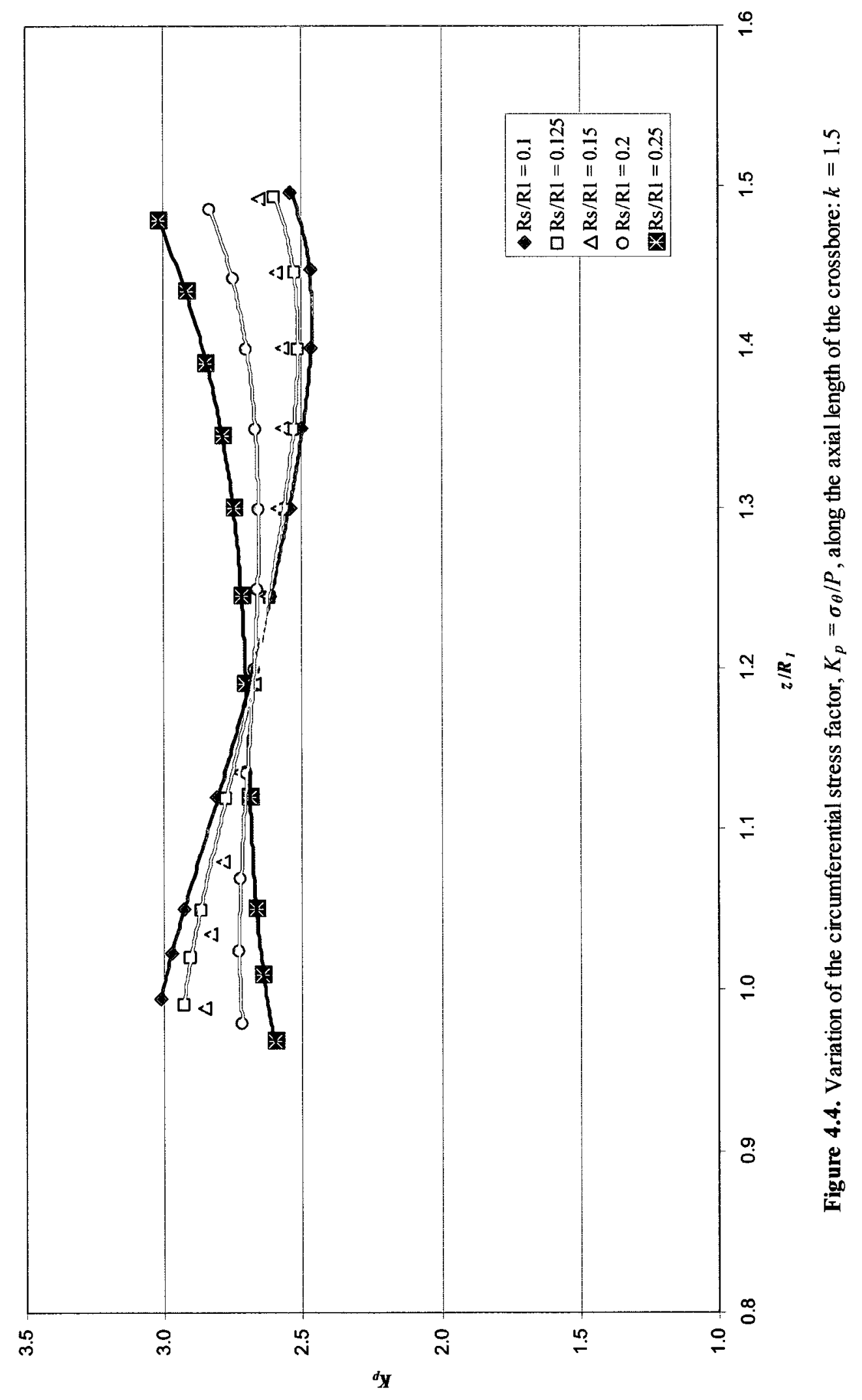




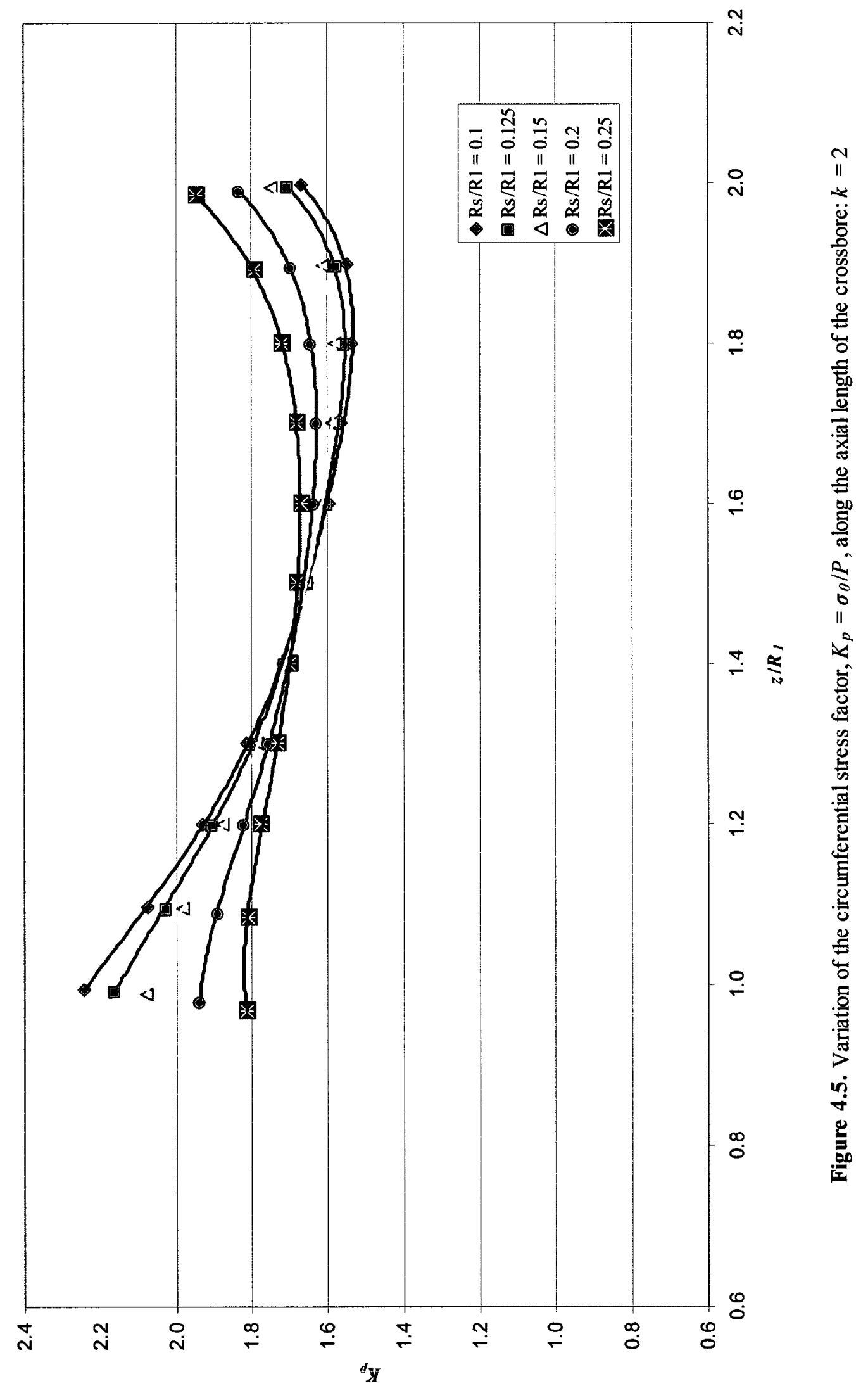




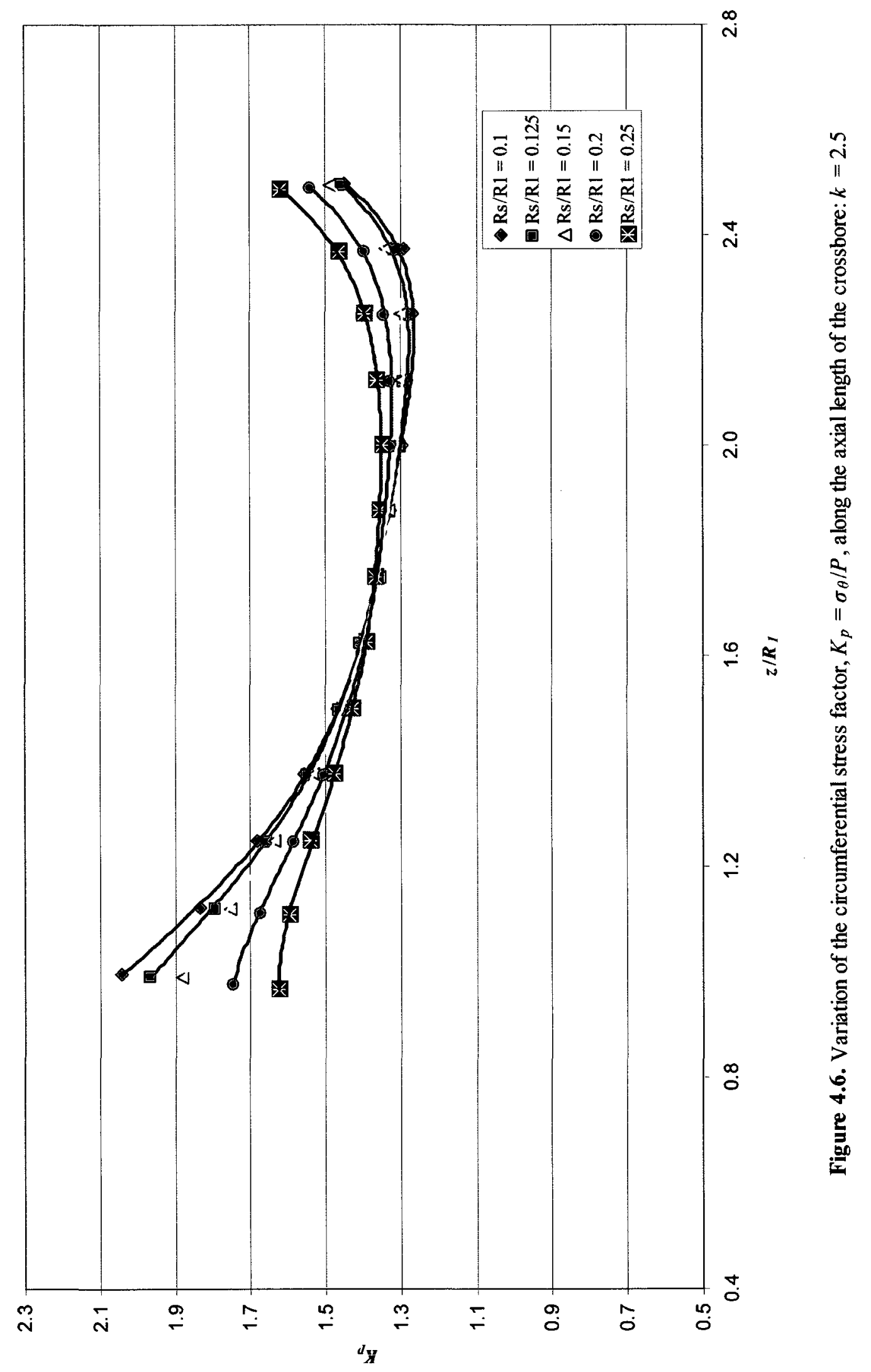




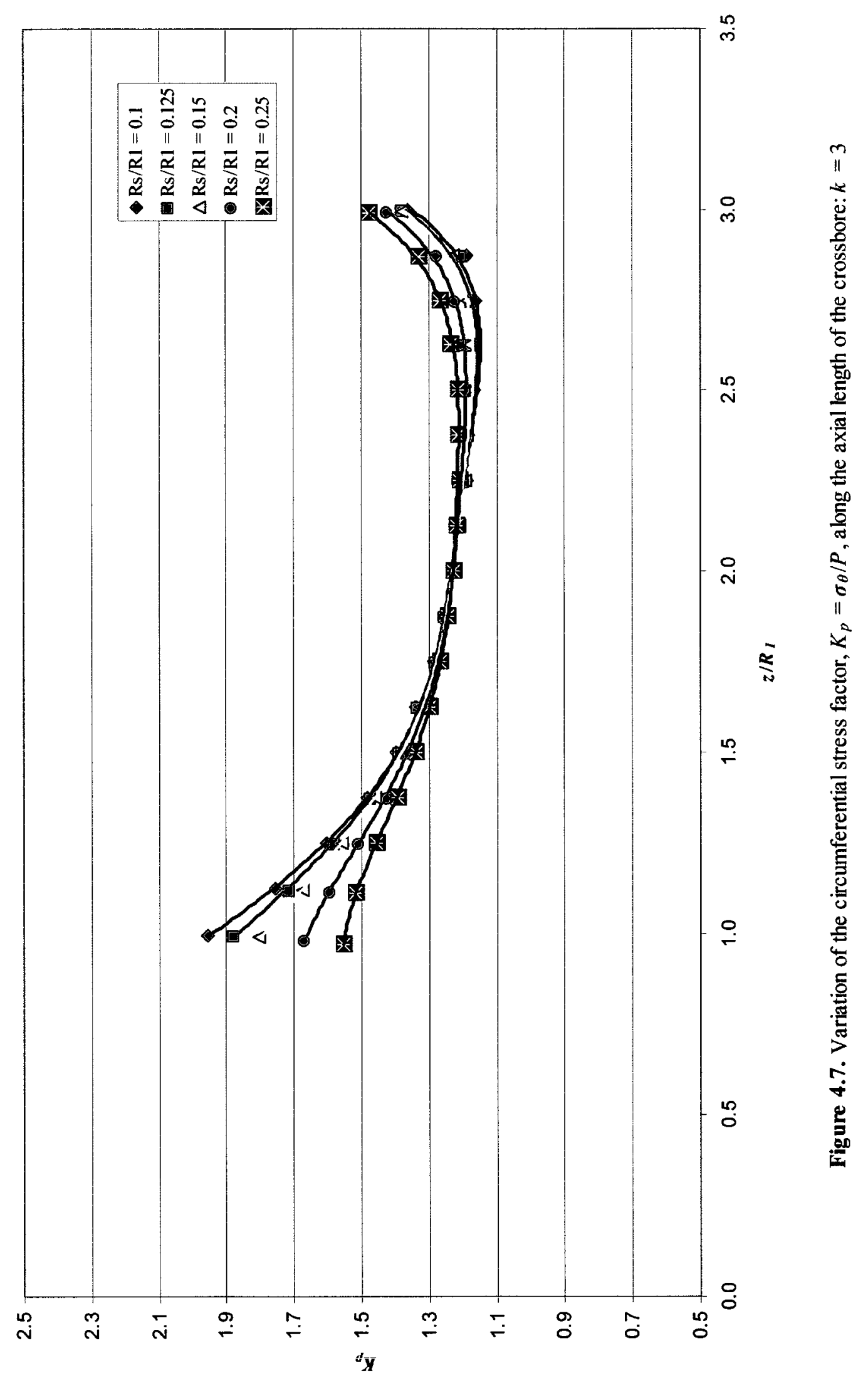




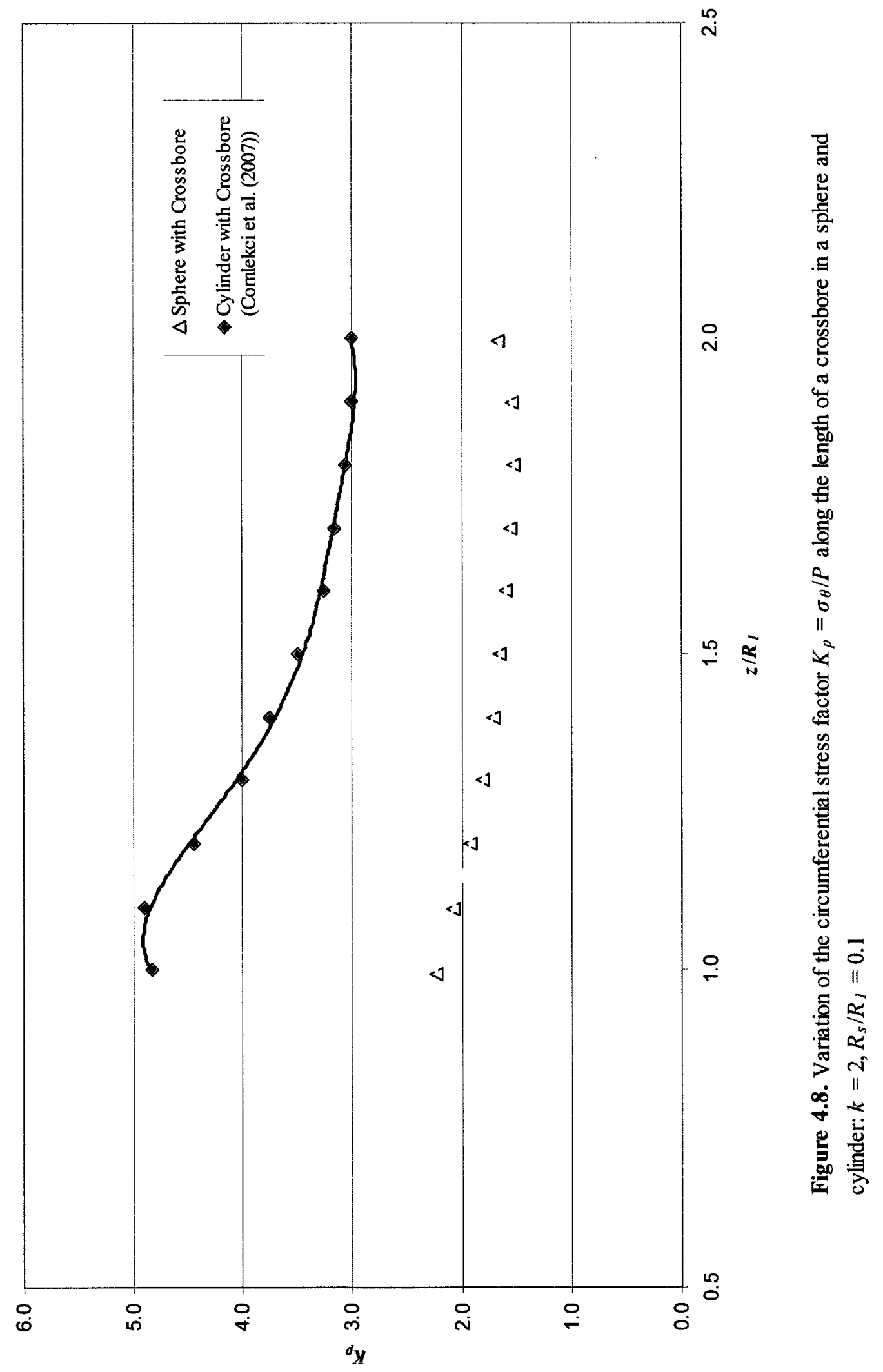




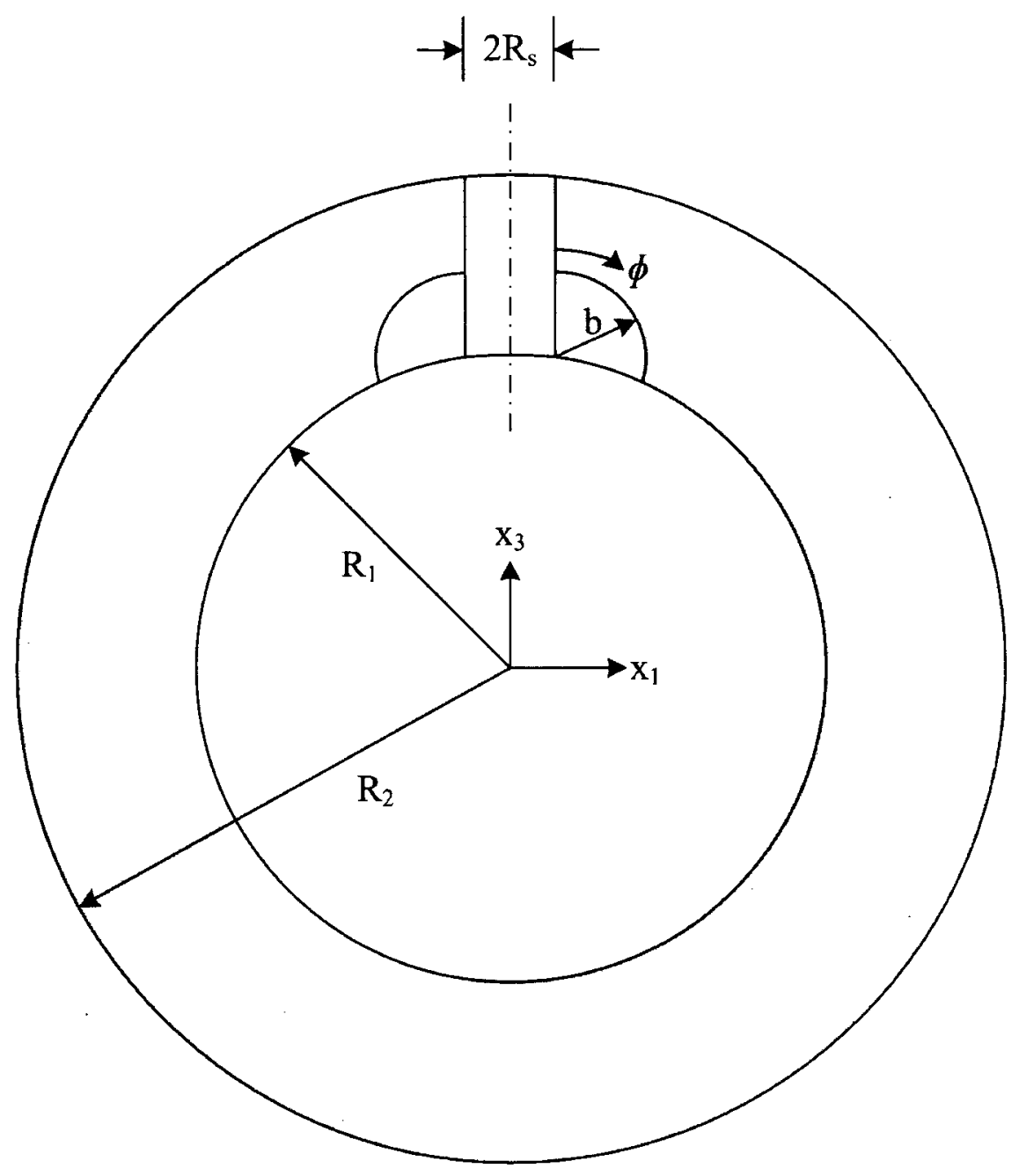

Figure 4.9. Diametrical section of a spherical vessel with a crossbore containing two cracks on the same plane 


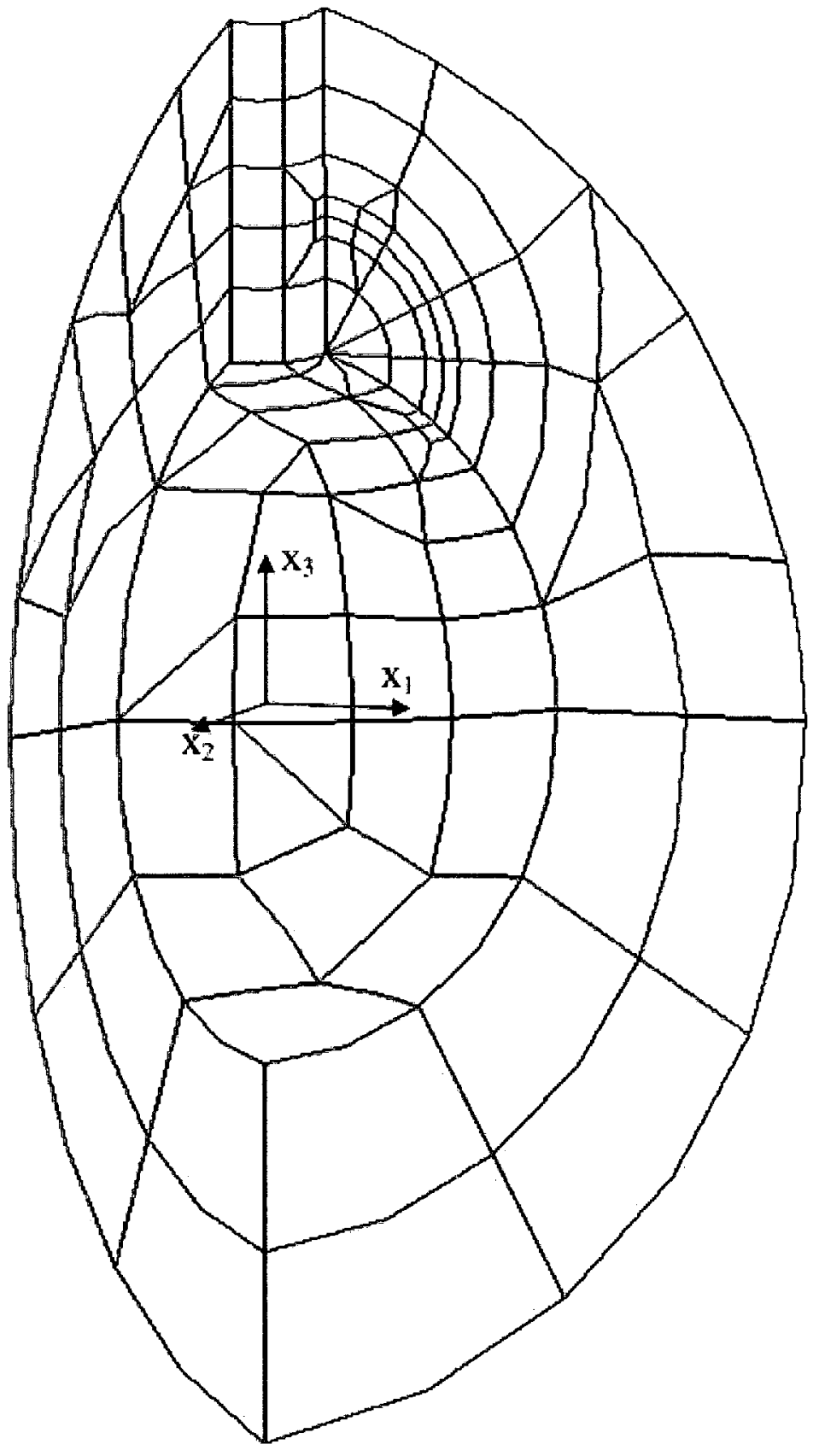

Figure 4.10. Three-dimensional BEM mesh of a spherical vessel with a crossbore containing two circular cracks on the same plane: $k=2, R_{S} / R_{I}=0.2, b / W=0.4$ 


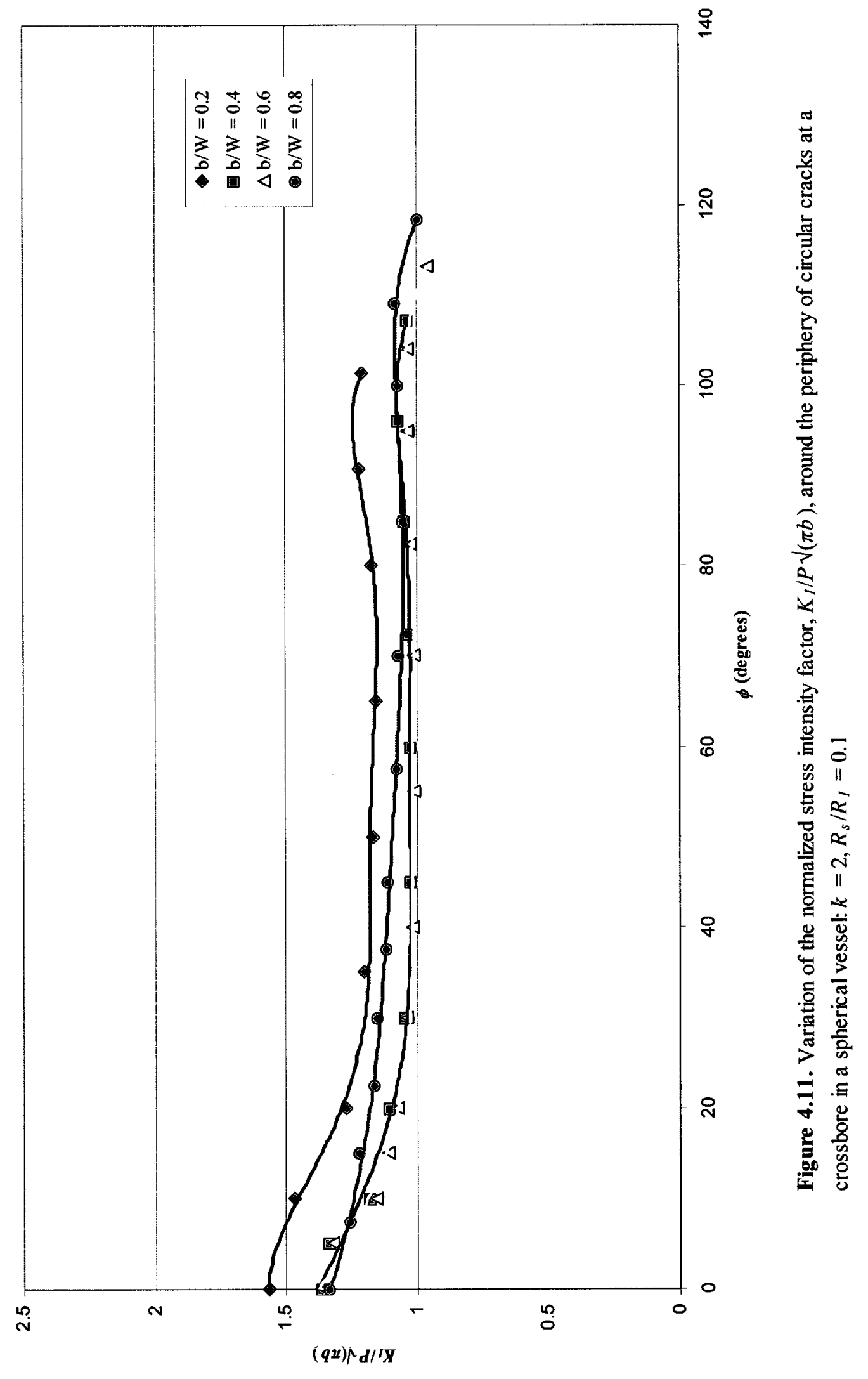




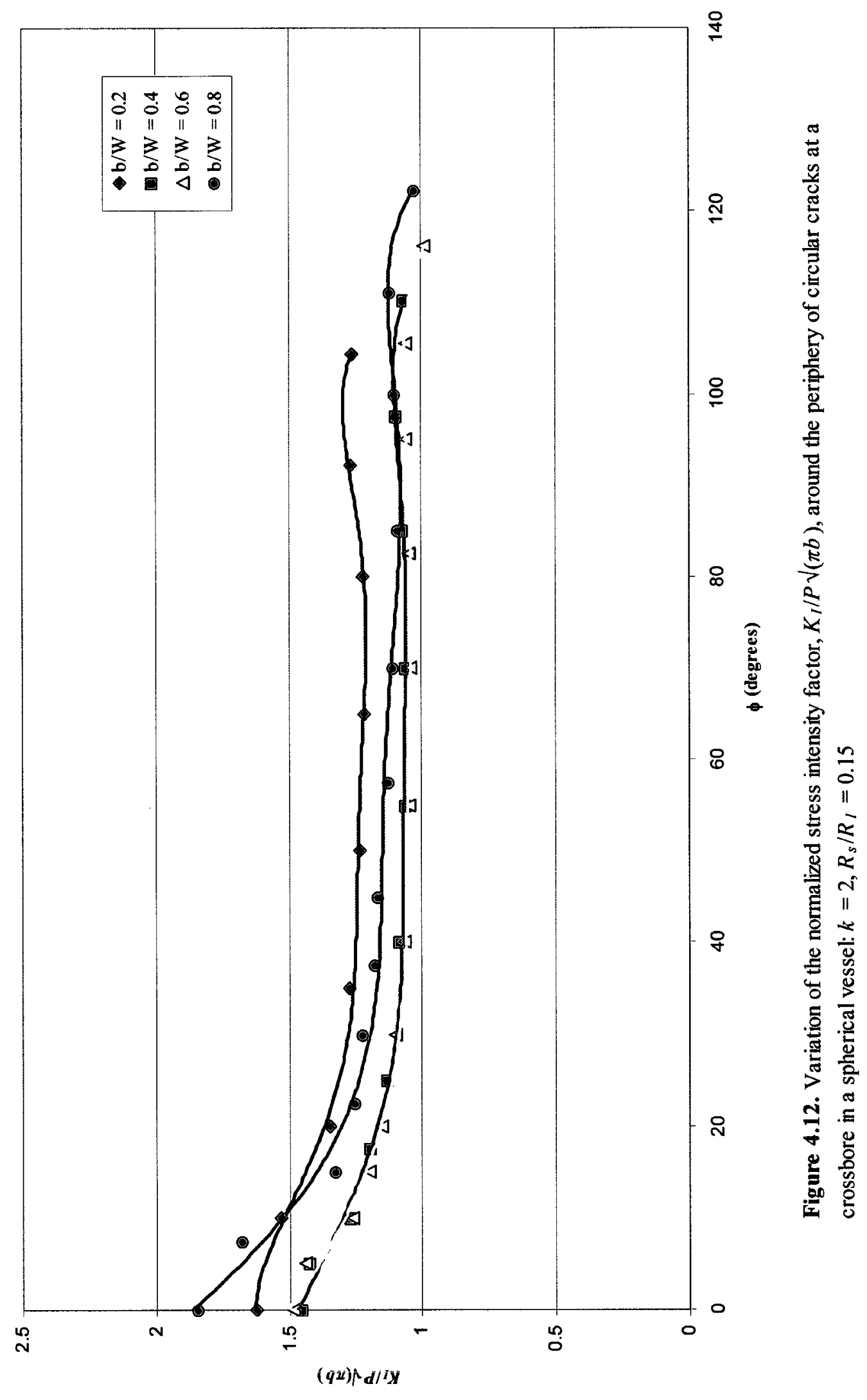




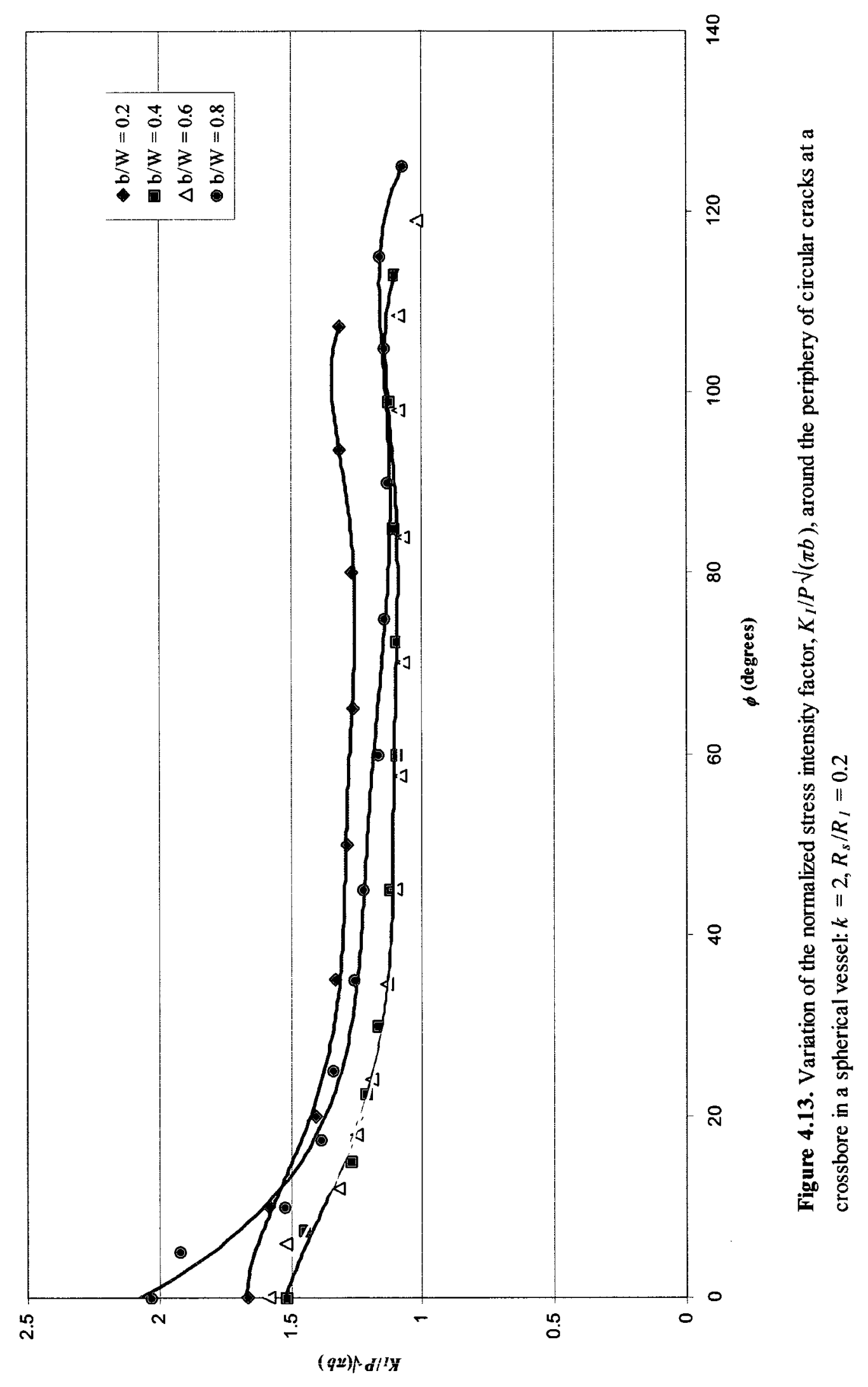




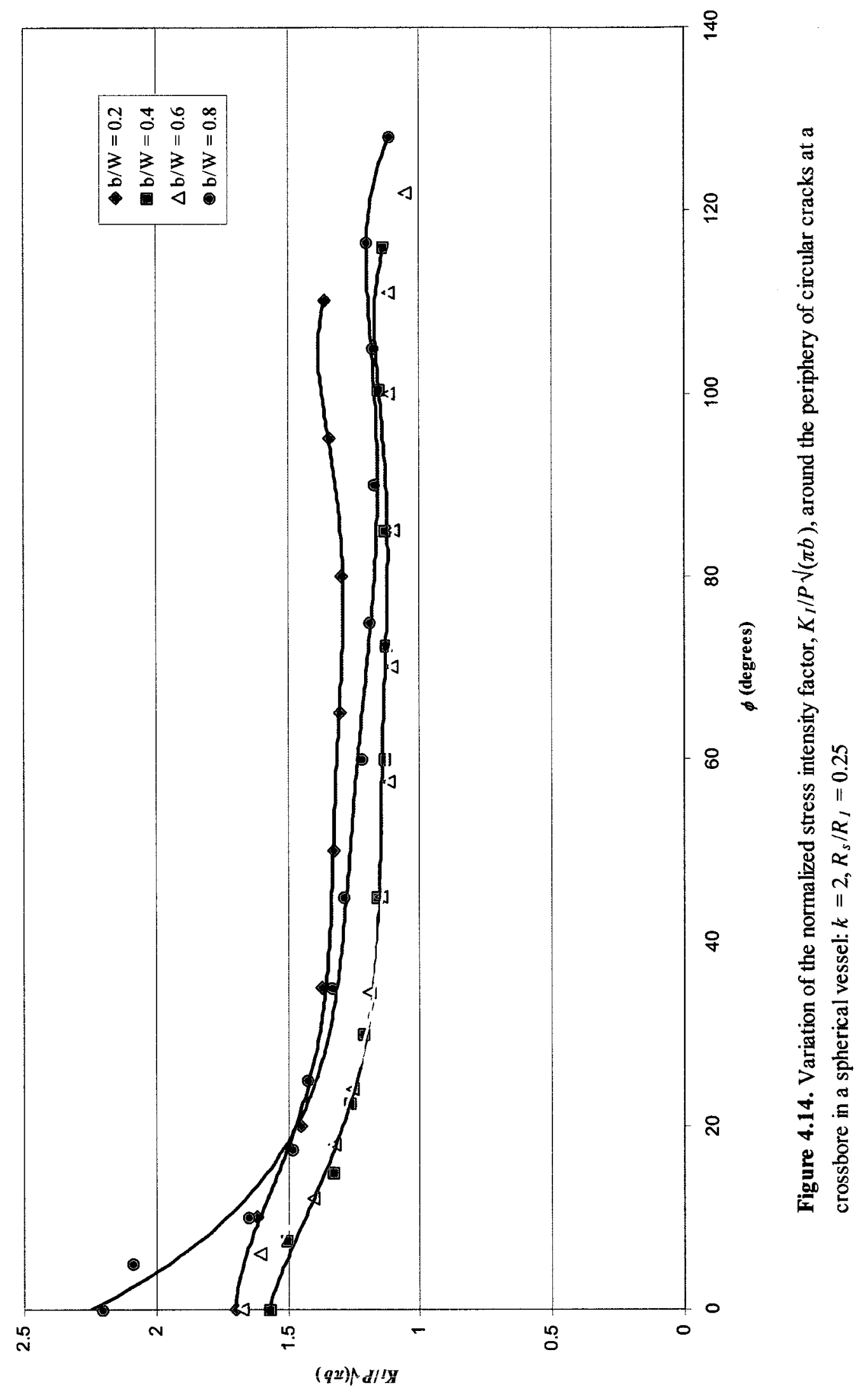




\section{Chapter 5}

\section{Conclusions}

The boundary element method (BEM) has been employed for the elastic stress analysis of thick-walled spherical pressure vessels with surface cracks. The cases treated also included spheres with a radial crossbore. The formulation of the method was first reviewed, and relevant examples have been presented to demonstrate its ability at solving stress concentration and linear elastic fracture problems accurately and efficiently.

The problem of an internal surface crack in an internally pressurized spherical vessel was investigated by analyzing a number of geometric combinations, namely, radius ratios, $k=$ $1.5,2,2.5,3$, crack aspect ratios, $b / a=1,0.8,0.6$, and relative crack depths, $b / W=0.2$, $0.4,0.6,0.8$. Normalized stress intensity factors have been determined along the periphery of the crack, $\phi$, for each case. Relative crack depths $b / W=0.2$ and 0.8 generally produced higher values compared to 0.4 and 0.6. For all cases, the normalized stress intensity factor remained relatively constant around the crack periphery until the free surface was approached. In proximity to this location, it was observed that the normalized stress intensity factor varied differently, depending on the relative crack depth and crack aspect ratio. Smaller semi-circular cracks had a gradual increase, while the normalized stress intensity factor for a large semi-circular crack decreased towards the free surface. Lowering the aspect ratio of the crack generally resulted in a less gradual variation, with rapid increases near the free surface. Polynomial influence coefficients were also obtained for each geometric combination. This is to allow other load cases with the same cracked geometry to be analyzed without performing an entirely new BEM analysis by invoking the influence function method. These were validated by using them to determine the normalized stress intensity factors, and comparing them to those that were obtained directly.

Stress analyses have also been performed on internally pressurized spherical vessels with a crossbore. The relative crossbore radii considered were, $R_{s} / R_{l}=0.1,0.125,0.15,0.2$, 0.25 . The normalized hoop stress, $\sigma_{\sigma} / P$, represented by the circumferential stress factor $K_{p}$, was determined along the axial length of the crossbore. It was determined that for the 
relatively smaller crossbores, the maximum circumferential stress factor occurred at the intersection of the crossbore and the internal surface of the sphere. However, it was shown that if the sphere contained a relatively large crossbore, $R_{s} / R_{l}>0.2$, the maximum circumferential stress factor could move to the exterior surface. Crossbores and other stress concentrations are typical sites of crack initiation. Therefore, a representative set of the possible geometries of a spherical vessel with cracks residing at its crossbore was investigated. The cracks were assumed to be situated on the same plane and of the circular form. The sphere radius ratio considered was $k=2$, with crossbore radii, $R_{S} / R_{I}=$ $0.1,0.15,0.2,0.25$, and relative crack depths, $b / W=0.2,0.4,0.6,0.8$. Normalized stress intensity factors have been determined along the crack front. It was observed that the maximum normalized stress intensity factor occurred where the crack intersected the crossbore. At some distance away from this location, the variation resembled that of the cracked spherical vessel without a crossbore. 


\section{References}

Adibi-Asi R. (2007), Autofrettaged Spherical Pressure Vessels Design, ASME Pressure Vessels and Piping Division Conference

Anderson T.L. (2005), Fracture Mechanics Fundamentals and Applications, $3^{\text {rd }}$ Edition, Taylor and Francis.

Atluri S.N. and Kathiresan K. (1979), Three-Dimensional Analyses of Surface Flaws in Thick-Walled Reactor Pressure Vessels using Displacement-Hybrid Finite Element Method, Nuclear Engineering and Design, 51, 163-176.

Becker A.A. (1992), The Boundary Element Method in Engineering: A Complete Course, Mcgraw-Hill.

Blackburn W.S. \& Hellen T.K. (1977), Calculation of Stress Intensity Factors in ThreeDimensions by the Finite Element Method, International Journal for Numerical Methods in Engineering, 11, 211-229.

Cruse T.A. (1969), Numerical Solutions in Three-Dimensional Elastostatics, International Journal of Solids and Structures, 5, 1259-1274.

Comlekci T., Mackenzie D., Hamilton R. \& Wood J. ( 2007), Elastic Stress Concentration at Radial Crossholes in Pressurized Thick Cylinders, Journal of Strain Analysis, 42.

Fenner R.T., Nadiri F. (1985), On the Use of Elliptical Side Branches to Thick-Walled Cylinders, International Journal of Pressure Vessels and Piping, 20, 139-154. 
Liu S.B. \& Tan C.L. (1995), Three-Dimensional Contact Mechanics Analysis of Crack Problems Using the Boundary Element Method, International Journal of Fracture, 72, 3967.

Luchi M.L. \& Poggialini A. (1983), Computation of Three-Dimensional Stress Intensity Factors Using Special Boundary Elements, Boundary Element Method International Conference, Hiroshima, 461-470.

Luchi M.L. \& Rizzuti S. (1987), Boundary Elements for Three-Dimensional Elastic Crack Analysis, International Journal for Numerical Methods in Engineering, 24, 22532271.

Makulsawatudom P., Mackenzie D. \& Hamilton R. (2004), Stress Concentration at Crossholes in Thick Cylindrical Vessels, Journal of Strain Analysis, 39.

Manning W.R. and Labrow S. (1974), High Pressure Engineering, Leonard-Hill Books.

Martinez J. and Dominguez J. (1984), On the Use of Quarter-Point Boundary Elements for Stress Intensity Factor Computations, International Journal for Numerical Methods in Engineering, 20, 1941-1950.

Masu L.M. (1997), Crossbore Configuration and Size Effects on the Stress Distribution in Thick-Walled Cylinders, International Journal of Pressure Vessels and Piping, 171-176.

Masu L.M. (1998), Numerical Analysis of Cylinders Containing Circular Offset Crossbores, International Journal of Pressure Vessels and Piping, 191-196.

Parker A. \& Huang X. (2007), Autofrettage of a Spherical Pressure Vessel, ASME Pressure Vessels and Piping Division Conference. 
Perl M. (2008), Thermal Simulation of an Arbitrary Residual Stress Field in a Fully or Partially Autofrettaged Thick-Walled Spherical Pressure Vessel, Journal of Pressure Vessel Technology, 130.

Plante K. (2008), Boundary Element Analysis of Curved Tubing with a Semi-Elliptical Crack, M.A.Sc. Thesis, Carleton University.

Tan C.L. (1983), Interior Point Solutions for Boundary Integral Equation Axisymmetric Stress Analysis, Applied Mathematical Modelling, 8, 57-60

Tan C.L. (1986), Stress Redistributions in Thick-Walled Cylinders Due to the Introduction of a Crossbore After Autofrettage, Journal of Strain Analysis, 21.

Tan C.L. \& Fenner R.T. (1979), Elastic Fracture Mechanics Analysis by the Boundary Integral Equation Method, Proceedings of the Royal Society of London. Series A, Mathematical and Physical Sciences, Volume 369, 243-260.

Tan C.L. \& Fenner R.T. (1980), Boundary Integral Equation Stress Analysis of Some Problems in High Pressure Engineering, The Second International Symposium on Innovative Numerical Analysis in Applied Engineering Science, Montreal Canada.

Tan C.L. \& Fenner R.T. (1980), Stress Intensity Factors for Semi-Elliptical Surface Cracks in Pressurized Cylinders Using the Boundary Integral Equation Method, International Journal of Fracture, 16.

Tan C.L. \& Selvaduri A.P.S. (1986), Internally Indented Penny-Shaped Cracks: A Comparison of Analytical and Boundary Integral Equation Estimates, Theoretical and Applied Fracture Mechanics, 5, 181-188. 
Tan C.L. \& Shim M.L. (1986), Stress Intensity Factor Influence Coefficients for Internal Surface Cracks in Thick-Walled Cylinders, International Journal of Pressure Vessels and Piping, 24, 49-72.

Tanaka M. \& Itoh H. (1987), New Crack Elements Applied to Boundary Element Computation of Stress Intensity Factors in Three-Dimensional Elastostatics, Role of Fracture Mechanics in Modern Technology.

Wang Q.Z. (2002), Simple Formulae for the Stress Concentration Factor for Two and Three-Dimensional Holes in Finite Domains, Journal of Strain Analysis, 37. 


\section{Appendix A}

\section{Axisymmetric Displacement and Traction Solutions for Kelvin's Problem of a Point Force in an Infinite Body}

The axisymmetric fundamental solutions, $U_{i j}(p, q)$ and $T_{i j}(p, q)(i, j=r, z)$, are defined below. They represent the displacement and traction at a field point $q$, with coordinates $\left(x_{q r}, x_{q z}\right)$, due to a unit load at point $p$, with coordinates $\left(x_{p r}, \mathrm{x}_{\mathrm{pz}}\right)$.

$$
\begin{aligned}
& U_{r r}(p, q)=\varphi_{1} K+\varphi_{2} I \\
& U_{r=}(p, q)=\varphi_{3} K+\varphi_{4} I \\
& U_{z r}(p, q)=\varphi_{5} K+\varphi_{6} I \\
& U_{z=}(p, q)=\varphi_{7} K+\varphi_{8} I \\
& T_{r r}(p, q)=2 G\left\{\left[B_{1} n_{r}+B_{2} n_{z}\right] K+\left[C_{1} n_{r}+C_{2} n_{z}\right] I\right\} \\
& T_{r=}(p, q)=2 G\left\{\left[B_{3} n_{r}+B_{4} n_{z}\right] K+\left[C_{3} n_{r}+C_{4} n_{z}\right] I\right\} \\
& T_{z r}(p, q)=2 G\left\{\left[B_{5} n_{r}+B_{6} n_{z}\right] K+\left[C_{5} n_{r}+C_{6} n_{z}\right] I\right\} \\
& T_{z=}(p, q)=2 G\left\{\left[B_{7} n_{r}+B_{8} n_{z}\right] K+\left[C_{7} n_{r}+C_{8} n_{z}\right] I\right\}
\end{aligned}
$$

Here the shear modulus is denoted by $G$, and $K(m, \pi / 2)$ and $I(m, \pi / 2)$ are the complete elliptical integrals of the first and second kind, abbreviated by $K$ and $I$. The modulus $m$ is

$$
m=\frac{2 \sqrt{x_{q r} x_{p r}}}{A}
$$

where

$$
\begin{aligned}
& A=\left[\left(x_{p r}+x_{q r}\right)^{2}+\bar{Z}^{2}\right]^{1 / 2} \\
& \bar{Z}=\left(x_{p=}-x_{q=}\right)
\end{aligned}
$$

Also, $\rho$ is the distance between $p$ and $q$. Therefore,

$$
\rho^{2}=\left[\left(x_{p r}-x_{q r}\right)^{2}+\bar{Z}^{2}\right]
$$


Also,

$$
\alpha=\frac{1}{16 \pi^{2} \mu(1-v)}
$$

and $n_{r}$ and $n_{z}$ are the radial and axial components of the unit outward normal at point $q$. Finally, the remaining expressions for the fundamental solutions are below.

$$
\begin{aligned}
& M=\left(x_{p r}{ }^{2}+x_{q r}{ }^{2}+\bar{Z}^{2}\right) \quad N=\left(x_{p r}{ }^{2}-x_{q r}{ }^{2}+\bar{Z}^{2}\right) \\
& \varphi_{1}=\frac{\alpha}{x_{p r} x_{q r} A}\left[(3-4 v) M+\bar{Z}^{2}\right] \quad \varphi_{2}=\frac{-\alpha}{x_{p r} x_{q r} A}\left[(3-4 v) A^{2}+\frac{M \bar{Z}^{2}}{\rho^{2}}\right] \\
& \varphi_{3}=\frac{\alpha \bar{Z}}{x_{p r} A} \quad \varphi_{4}=\frac{-\alpha \bar{Z}}{x_{p r} \rho^{2} A}\left(M-2 x_{p r}{ }^{2}\right) \\
& \varphi_{5}=\frac{-\alpha \bar{Z}}{x_{q r} A} \\
& \varphi_{6}=\frac{\alpha \bar{Z}}{x_{q r} \rho^{2} A} N \\
& \varphi_{7}=\frac{2 \alpha}{A}(3-4 v) \\
& \varphi_{8}=\frac{2 \alpha}{\rho^{2} A} \bar{Z}^{2} \\
& B_{1}=\frac{\alpha}{2 x_{p r} x_{q r}{ }^{2} A}\left[3\left(x_{q r}{ }^{2}-\bar{Z}^{2}\right)-(3-4 v)\left(M+N+x_{q r}{ }^{2}\right)+\frac{M N \bar{Z}^{2}}{\rho^{2} A^{2}}\right] \\
& B_{2}=\frac{\alpha \bar{Z}}{x_{p r} x_{q r} \rho^{2} A}\left[(2 v-3) \rho^{2}+\frac{M \bar{Z}^{2}}{A^{2}}\right] \\
& B_{3}=B_{2} \\
& B_{4}=\frac{\alpha}{x_{p r} \rho^{2} A}\left[(2 v-1) \rho^{2}+\frac{\left(M-2 x_{p r}{ }^{2}\right)}{A^{2}} \bar{Z}^{2}\right] \\
& B_{5}=\frac{\alpha \bar{Z}}{\rho^{2} A}\left[\frac{\rho^{2}}{x_{q r}{ }^{2}}+\frac{2 \bar{Z}^{2}}{A^{2}}\right] \\
& B_{6}=\frac{\alpha}{x_{q r} \rho^{2} A}\left[(2 v-1) \rho^{2}-\frac{N \bar{Z}^{2}}{A^{2}}\right] \\
& B_{7}=B_{6} \\
& B_{8}=\frac{-2 \alpha \bar{Z}^{3}}{\rho^{2} A^{3}}
\end{aligned}
$$




$$
\begin{aligned}
C_{1} & =\frac{\alpha}{x_{p r} x_{q r}{ }^{2} \rho^{2} A}\left[(3-4 v)\left(x_{p r}{ }^{2}+\bar{Z}^{2}\right) N+3 \bar{Z}^{2}\left(M+x_{q r}{ }^{2}\right)\right. \\
& \left.-2 v x_{q r}{ }^{2}\left(5 \bar{Z}^{2}-x_{p r}{ }^{2}+x_{q r}{ }^{2}\right)-\frac{2 M^{2} N \bar{Z}^{2}}{\rho^{2} A^{2}}\right] \\
C_{2} & =\frac{\alpha \bar{Z}}{x_{p r} x_{q r} \rho^{2} A}\left[\bar{Z}^{2}\left(3-\frac{4 M^{2}}{\rho^{2} A^{2}}\right)+(3-2 v) M\right] \\
C_{3} & =C_{2} \\
C_{4} & =\frac{\alpha}{x_{p r} \rho^{2} A}\left[(1-2 v)\left(M-2 x_{p r}{ }^{2}\right)+\bar{Z}^{2}\left(\frac{8 N x_{p r}{ }^{2}}{\rho^{2} A^{2}}-1\right)\right] \\
C_{5} & =\frac{\alpha \bar{Z}}{\rho^{2} A}\left[(3+4 v)-\frac{\left(x_{p r}{ }^{2}+\bar{Z}^{2}\right)}{x_{q r}{ }^{2}}-\frac{8 M \bar{Z}^{2}}{\rho^{2} A^{2}}\right] \\
C_{6} & =\frac{\alpha}{x_{q r} \rho^{2} A}\left[(1-2 v) N-3 \bar{Z}^{2}+\frac{4 M N \bar{Z}^{2}}{\rho^{2} A^{2}}\right] \\
C_{7} & =C_{6} \\
C_{8} & =\frac{2 \alpha \bar{Z}}{\rho^{2} A^{2}}\left[(1-2 v)+\frac{4 M \bar{Z}^{2}}{\rho^{2} A^{2}}\right]
\end{aligned}
$$




\section{Appendix B}

\section{Shape Functions}

\section{B.1 Shape Functions for Quadrilateral Elements}

The shape functions for a quadratic quadrilateral element as shown in Figure 2.1 are given below.

$$
\begin{aligned}
& N^{1}\left(\xi_{1}, \xi_{2}\right)=-\frac{1}{4}\left(1-\xi_{1}\right)\left(1-\xi_{2}\right)\left(1+\xi_{1}+\xi_{2}\right) \\
& N^{2}\left(\xi_{1}, \xi_{2}\right)=\frac{1}{2}\left(1-\xi_{1}^{2}\right)\left(1-\xi_{2}\right) \\
& N^{3}\left(\xi_{1}, \xi_{2}\right)=-\frac{1}{4}\left(1+\xi_{1}\right)\left(1-\xi_{2}\right)\left(1-\xi_{1}+\xi_{2}\right) \\
& N^{4}\left(\xi_{1}, \xi_{2}\right)=\frac{1}{2}\left(1-\xi_{1}\right)\left(1-\xi_{2}^{2}\right) \\
& N^{5}\left(\xi_{1}, \xi_{2}\right)=\frac{1}{2}\left(1+\xi_{1}\right)\left(1-\xi_{2}^{2}\right) \\
& N^{6}\left(\xi_{1}, \xi_{2}\right)=-\frac{1}{4}\left(1-\xi_{1}\right)\left(1+\xi_{2}\right)\left(1+\xi_{1}-\xi_{2}\right) \\
& N^{7}\left(\xi_{1}, \xi_{2}\right)=\frac{1}{2}\left(1-\xi_{1}^{2}\right)\left(1+\xi_{2}\right) \\
& N^{8}\left(\xi_{1}, \xi_{2}\right)=-\frac{1}{4}\left(1+\xi_{1}\right)\left(1+\xi_{2}\right)\left(1-\xi_{1}-\xi_{2}\right)
\end{aligned}
$$




\section{B.2 Shape Functions for Triangular Elements}

The shape functions for a quadratic triangular element as shown in Figure 2.2 are given below.

$$
\begin{aligned}
& N^{1}\left(\xi_{1}, \xi_{2}\right)=\left(1-\xi_{1}-\xi_{2}\right)\left(1-2 \xi_{1}-2 \xi_{2}\right) \\
& N^{2}\left(\xi_{1}, \xi_{2}\right)=4 \xi_{1}\left(1-\xi_{1}-\xi_{2}\right) \\
& N^{3}\left(\xi_{1}, \xi_{2}\right)=\xi_{1}\left(2 \xi_{1}-1\right) \\
& N^{4}\left(\xi_{1}, \xi_{2}\right)=4 \xi_{1} \xi_{2} \\
& N^{5}\left(\xi_{1}, \xi_{2}\right)=\xi_{2}\left(2 \xi_{2}-1\right) \\
& N^{6}\left(\xi_{1}, \xi_{2}\right)=4 \xi_{2}\left(1-\xi_{1}-\xi_{2}\right)
\end{aligned}
$$

\section{B.3 Shape Functions for Axisymmetric Line Elements}

The shape functions for a quadratic line element as shown in Figure 2.3 are given below.

$$
\begin{aligned}
& N^{\prime}(\xi)=-\frac{1}{2} \xi(1-\xi) \\
& N^{2}(\xi)=\left(1-\xi^{2}\right) \\
& N^{3}(\xi)=\frac{1}{2} \xi(1+\xi)
\end{aligned}
$$




\section{B.4 Shape Functions for Crack-Front Elements}

The displacement and traction shape functions for the quadrilateral element shown in Figure 2.1 are as follows if nodes 1-2-3 are along the crack front.

$$
\begin{aligned}
& N_{d}^{1}\left(\xi_{1}, \xi_{2}\right)=\frac{1}{2}\left(1-\xi_{1}\right)\left[-\xi_{1}-\left(1-\frac{\sqrt{2}}{2} \xi_{1}\right) \sqrt{1+\xi_{2}}+\frac{\sqrt{2}}{2}\left(1+\xi_{2}\right)\right] \\
& N_{d}^{2}\left(\xi_{1}, \xi_{2}\right)=\left(1-\xi_{1}^{2}\right)\left[1-\frac{\sqrt{2}}{2} \sqrt{1+\xi_{2}}\right] \\
& N_{d}^{3}\left(\xi_{1}, \xi_{2}\right)=\frac{1}{2}\left(1+\xi_{1}\right)\left[\xi_{1}-\left(1+\frac{\sqrt{2}}{2} \xi_{1}\right) \sqrt{1+\xi_{2}}+\frac{\sqrt{2}}{2}\left(1+\xi_{2}\right)\right] \\
& N_{d}^{4}\left(\xi_{1}, \xi_{2}\right)=\frac{1}{2}\left(1+\xi_{1}\right)\left[(2+\sqrt{2}) \sqrt{1+\xi_{2}}-(1+\sqrt{2})\left(1+\xi_{2}\right)\right] \\
& N_{d}^{5}\left(\xi_{1}, \xi_{2}\right)=\frac{1}{4}\left(1+\xi_{1}\right)\left[\sqrt{2}\left(\xi_{1}-2-\sqrt{2}\right) \sqrt{1+\xi_{2}}+(2+\sqrt{2})\left(1+\xi_{2}\right)\right] \\
& N_{d}^{6}\left(\xi_{1}, \xi_{2}\right)=\frac{\sqrt{2}}{2}\left(1-\xi_{1}^{2}\right) \sqrt{1+\xi_{2}} \\
& N_{d}^{7}\left(\xi_{1}, \xi_{2}\right)=\frac{1}{4}\left(1-\xi_{1}\right)\left[-\sqrt{2}\left(\xi_{1}+2+\sqrt{2}\right) \sqrt{1+\xi_{2}}+(2+\sqrt{2})\left(1+\xi_{2}\right)\right] \\
& N_{d}^{8}\left(\xi_{1}, \xi_{2}\right)=\frac{1}{2}\left(1-\xi_{1}\right)\left[(2+\sqrt{2}) \sqrt{1+\xi_{2}}-(1+\sqrt{2})\left(1+\xi_{2}\right)\right]
\end{aligned}
$$

$$
\begin{aligned}
& N_{t}^{1}\left(\xi_{1}, \xi_{2}\right)=N_{d}^{1}\left(\xi_{1}, \xi_{2}\right) / \sqrt{1+\xi_{2}} \\
& N_{t}^{2}\left(\xi_{1}, \xi_{2}\right)=N_{d}^{2}\left(\xi_{1}, \xi_{2}\right) / \sqrt{1+\xi_{2}} \\
& N_{t}^{3}\left(\xi_{1}, \xi_{2}\right)=N_{d}^{3}\left(\xi_{1}, \xi_{2}\right) / \sqrt{1+\xi_{2}} \\
& N_{t}^{4}\left(\xi_{1}, \xi_{2}\right)=N_{d}^{4}\left(\xi_{1}, \xi_{2}\right) / \sqrt{1+\xi_{2}} \\
& N_{t}^{j}\left(\xi_{1}, \xi_{2}\right)=\sqrt{2} N_{d}^{5}\left(\xi_{1}, \xi_{2}\right) / \sqrt{1+\xi_{2}} \\
& N_{t}^{6}\left(\xi_{1}, \xi_{2}\right)=\sqrt{2} N_{d}^{6}\left(\xi_{1}, \xi_{2}\right) / \sqrt{1+\xi_{2}} \\
& N_{t}^{7}\left(\xi_{1}, \xi_{2}\right)=\sqrt{2} N_{d}^{7}\left(\xi_{1}, \xi_{2}\right) / \sqrt{1+\xi_{2}} \\
& N_{t}^{8}\left(\xi_{1}, \xi_{2}\right)=N_{d}^{8}\left(\xi_{1}, \xi_{2}\right) / \sqrt{1+\xi_{2}}
\end{aligned}
$$


The shape functions are as follows if nodes 3-4-5 are along the crack front.

$$
\begin{aligned}
& N_{d}^{1}\left(\xi_{1}, \xi_{2}\right)=\frac{1}{4}\left(1-\xi_{2}\right)\left[-\sqrt{2}\left(\xi_{2}+2+\sqrt{2}\right) \sqrt{1-\xi_{1}}+(2+\sqrt{2})\left(1-\xi_{1}\right)\right] \\
& N_{d}^{2}\left(\xi_{1}, \xi_{2}\right)=\frac{1}{2}\left(1-\xi_{2}\right)\left[(2+\sqrt{2}) \sqrt{1-\xi_{1}}-(1+\sqrt{2})\left(1-\xi_{1}\right)\right] \\
& N_{d}^{3}\left(\xi_{1}, \xi_{2}\right)=\frac{1}{2}\left(1-\xi_{2}\right)\left[-\xi_{2}-\left(1-\frac{\sqrt{2}}{2} \xi_{2}\right) \sqrt{1-\xi_{1}}+\frac{\sqrt{2}}{2}\left(1-\xi_{1}\right)\right] \\
& N_{d}^{4}\left(\xi_{1}, \xi_{2}\right)=\left(1-\xi_{2}^{2}\right)\left[1-\frac{\sqrt{2}}{2} \sqrt{1-\xi_{1}}\right] \\
& N_{d}^{j}\left(\xi_{1}, \xi_{2}\right)=\frac{1}{2}\left(1+\xi_{2}\right)\left[\xi_{2}-\left(1+\frac{\sqrt{2}}{2} \xi_{2}\right) \sqrt{1-\xi_{1}}+\frac{\sqrt{2}}{2}\left(1-\xi_{1}\right)\right] \\
& N_{d}^{6}\left(\xi_{1}, \xi_{2}\right)=\frac{1}{2}\left(1+\xi_{2}\right)\left[(2+\sqrt{2}) \sqrt{1-\xi_{1}}-(1+\sqrt{2})\left(1-\xi_{1}\right)\right] \\
& N_{d}^{7}\left(\xi_{1}, \xi_{2}\right)=\frac{1}{4}\left(1+\xi_{2}\right)\left[\sqrt{2}\left(\xi_{2}-2-\sqrt{2}\right) \sqrt{1-\xi_{1}}+(2+\sqrt{2})\left(1-\xi_{1}\right)\right] \\
& N_{d}^{8}\left(\xi_{1}, \xi_{2}\right)=\frac{\sqrt{2}}{2}\left(1-\xi_{2}^{2}\right) \sqrt{1-\xi_{1}}
\end{aligned}
$$

$$
\begin{aligned}
& N_{(}^{1}\left(\xi_{1}, \xi_{2}\right)=\sqrt{2} N_{d}^{1}\left(\xi_{1}, \xi_{2}\right) / \sqrt{1-\xi_{1}} \\
& N_{t}^{2}\left(\xi_{1}, \xi_{2}\right)=N_{d}^{2}\left(\xi_{1}, \xi_{2}\right) / \sqrt{1-\xi_{1}} \\
& N_{,}^{3}\left(\xi_{1}, \xi_{2}\right)=N_{d}^{3}\left(\xi_{1}, \xi_{2}\right) / \sqrt{1-\xi_{1}} \\
& N_{,}^{4}\left(\xi_{1}, \xi_{2}\right)=N_{d}^{4}\left(\xi_{1}, \xi_{2}\right) / \sqrt{1-\xi_{1}} \\
& N_{t}^{s}\left(\xi_{1}, \xi_{2}\right)=N_{d}^{s}\left(\xi_{1}, \xi_{2}\right) / \sqrt{1-\xi_{1}} \\
& N_{t}^{6}\left(\xi_{1}, \xi_{2}\right)=N_{d}^{6}\left(\xi_{1}, \xi_{2}\right) / \sqrt{1-\xi_{1}} \\
& N_{t}^{7}\left(\xi_{1}, \xi_{2}\right)=\sqrt{2} N_{d}^{7}\left(\xi_{1}, \xi_{2}\right) / \sqrt{1-\xi_{1}} \\
& N_{t}^{8}\left(\xi_{1}, \xi_{2}\right)=\sqrt{2} N_{d}^{8}\left(\xi_{1}, \xi_{2}\right) / \sqrt{1-\xi_{1}}
\end{aligned}
$$


The shape functions are as follows if nodes 5-6-7 are along the crack front.

$$
\begin{aligned}
& N_{d}^{1}\left(\xi_{1}, \xi_{2}\right)=\frac{1}{4}\left(1-\xi_{1}\right)\left[-\sqrt{2}\left(\xi_{1}+2+\sqrt{2}\right) \sqrt{1-\xi_{1}}+(2+\sqrt{2})\left(1-\xi_{2}\right)\right] \\
& N_{d}^{2}\left(\xi_{1}, \xi_{2}\right)=\frac{\sqrt{2}}{2}\left(1-\xi_{1}^{2}\right) \sqrt{1-\xi_{2}} \\
& N_{d}^{3}\left(\xi_{1}, \xi_{2}\right)=\frac{1}{4}\left(1+\xi_{1}\right)\left[\sqrt{2}\left(\xi_{1}-2-\sqrt{2}\right) \sqrt{1-\xi_{2}}+(2+\sqrt{2})\left(1-\xi_{2}\right)\right] \\
& N_{d}^{4}\left(\xi_{1}, \xi_{2}\right)=\frac{1}{2}\left(1+\xi_{1}\right)\left[(2+\sqrt{2}) \sqrt{1-\xi_{2}}-(1+\sqrt{2})\left(1-\xi_{2}\right)\right] \\
& N_{d}^{5}\left(\xi_{1}, \xi_{2}\right)=\frac{1}{2}\left(1+\xi_{1}\right)\left[\xi_{1}-\left(1+\frac{\sqrt{2}}{2} \xi_{1}\right) \sqrt{1-\xi_{2}}+\frac{\sqrt{2}}{2}\left(1-\xi_{2}\right)\right] \\
& N_{d}^{6}\left(\xi_{1}, \xi_{2}\right)=\left(1-\xi_{1}^{2}\right)\left[1-\frac{\sqrt{2}}{2} \sqrt{1-\xi_{2}}\right] \\
& N_{d}^{7}\left(\xi_{1}, \xi_{2}\right)=\frac{1}{2}\left(1-\xi_{1}\right)\left[-\xi_{1}-\left(1-\frac{\sqrt{2}}{2} \xi_{1}\right) \sqrt{1-\xi_{2}}+\frac{\sqrt{2}}{2}\left(1-\xi_{2}\right)\right] \\
& N_{d}^{8}\left(\xi_{1}, \xi_{2}\right)=\frac{1}{2}\left(1-\xi_{1}\right)\left[(2+\sqrt{2}) \sqrt{1-\xi_{2}}-(1+\sqrt{2})\left(1-\xi_{2}\right)\right]
\end{aligned}
$$

$$
\begin{aligned}
& N_{t}^{1}\left(\xi_{1}, \xi_{2}\right)=\sqrt{2} N_{d}^{1}\left(\xi_{1}, \xi_{2}\right) / \sqrt{1-\xi_{2}} \\
& N_{1}^{2}\left(\xi_{1}, \xi_{2}\right)=\sqrt{2} N_{d}^{2}\left(\xi_{1}, \xi_{2}\right) / \sqrt{1-\xi_{2}} \\
& N_{t}^{3}\left(\xi_{1}, \xi_{2}\right)=\sqrt{2} N_{d}^{3}\left(\xi_{1}, \xi_{2}\right) / \sqrt{1-\xi_{2}} \\
& N_{t}^{4}\left(\xi_{1}, \xi_{2}\right)=N_{d}^{4}\left(\xi_{1}, \xi_{2}\right) / \sqrt{1-\xi_{2}} \\
& N_{1}^{5}\left(\xi_{1}, \xi_{2}\right)=N_{d}^{5}\left(\xi_{1}, \xi_{2}\right) / \sqrt{1-\xi_{2}} \\
& N_{t}^{6}\left(\xi_{1}, \xi_{2}\right)=N_{d}^{6}\left(\xi_{1}, \xi_{2}\right) / \sqrt{1-\xi_{2}} \\
& N_{,}^{7}\left(\xi_{1}, \xi_{2}\right)=N_{d}^{7}\left(\xi_{1}, \xi_{2}\right) / \sqrt{1-\xi_{2}} \\
& N_{,}^{8}\left(\xi_{1}, \xi_{2}\right)=N_{d}^{8}\left(\xi_{1}, \xi_{2}\right) / \sqrt{1-\xi_{2}}
\end{aligned}
$$


The shape functions are as follows if nodes 7-8-1 are along the crack front.

$$
\begin{aligned}
& N_{d}^{1}\left(\xi_{1}, \xi_{2}\right)=\frac{1}{2}\left(1-\xi_{2}\right)\left[-\xi_{2}-\left(1-\frac{\sqrt{2}}{2} \xi_{2}\right) \sqrt{1+\xi_{1}}+\frac{\sqrt{2}}{2}\left(1+\xi_{1}\right)\right] \\
& N_{d}^{2}\left(\xi_{1}, \xi_{2}\right)=\frac{1}{2}\left(1-\xi_{2}\right)\left[(2+\sqrt{2}) \sqrt{1+\xi_{1}}-(1+\sqrt{2})\left(1+\xi_{1}\right)\right] \\
& N_{d}^{3}\left(\xi_{1}, \xi_{2}\right)=\frac{1}{4}\left(1-\xi_{2}\right)\left[-\sqrt{2}\left(\xi_{2}+2+\sqrt{2}\right) \sqrt{1+\xi_{1}}+(2+\sqrt{2})\left(1+\xi_{1}\right)\right] \\
& N_{d}^{4}\left(\xi_{1}, \xi_{2}\right)=\frac{\sqrt{2}}{2}\left(1-\xi_{2}^{2}\right) \sqrt{1+\xi_{1}} \\
& N_{d}^{5}\left(\xi_{1}, \xi_{2}\right)=\frac{1}{4}\left(1+\xi_{2}\right)\left[\sqrt{2}\left(\xi_{2}-2-\sqrt{2}\right) \sqrt{1+\xi_{1}}+(2+\sqrt{2})\left(1+\xi_{1}\right)\right] \\
& N_{d}^{6}\left(\xi_{1}, \xi_{2}\right)=\frac{1}{2}\left(1+\xi_{2}\right)\left[(2+\sqrt{2}) \sqrt{1+\xi_{1}}-(1+\sqrt{2})\left(1+\xi_{1}\right)\right] \\
& N_{d}^{7}\left(\xi_{1}, \xi_{2}\right)=\frac{1}{2}\left(1+\xi_{2}\right)\left[\xi_{2}-\left(1+\frac{\sqrt{2}}{2} \xi_{2}\right) \sqrt{1+\xi_{1}}+\frac{\sqrt{2}}{2}\left(1+\xi_{1}\right)\right] \\
& N_{d}^{8}\left(\xi_{1}, \xi_{2}\right)=\left(1-\xi_{2}^{2}\right)\left[1-\frac{\sqrt{2}}{2} \sqrt{1+\xi_{1}}\right]
\end{aligned}
$$

$$
\begin{aligned}
& N_{t}^{1}\left(\xi_{1}, \xi_{2}\right)=N_{d}^{1}\left(\xi_{1}, \xi_{2}\right) / \sqrt{1+\xi_{1}} \\
& N_{t}^{2}\left(\xi_{1}, \xi_{2}\right)=N_{d}^{2}\left(\xi_{1}, \xi_{2}\right) / \sqrt{1+\xi_{1}} \\
& N_{t}^{3}\left(\xi_{1}, \xi_{2}\right)=\sqrt{2} N_{d}^{3}\left(\xi_{1}, \xi_{2}\right) / \sqrt{1+\xi_{1}} \\
& N_{t}^{4}\left(\xi_{1}, \xi_{2}\right)=\sqrt{2} N_{d}^{4}\left(\xi_{1}, \xi_{2}\right) / \sqrt{1+\xi_{1}} \\
& N_{t}^{5}\left(\xi_{1}, \xi_{2}\right)=\sqrt{2} N_{d}^{j}\left(\xi_{1}, \xi_{2}\right) / \sqrt{1+\xi_{1}} \\
& N_{t}^{6}\left(\xi_{1}, \xi_{2}\right)=N_{d}^{6}\left(\xi_{1}, \xi_{2}\right) / \sqrt{1+\xi_{1}} \\
& N_{t}^{7}\left(\xi_{1}, \xi_{2}\right)=N_{d}^{7}\left(\xi_{1}, \xi_{2}\right) / \sqrt{1+\xi_{1}} \\
& N_{t}^{8}\left(\xi_{1}, \xi_{2}\right)=N_{d}^{8}\left(\xi_{1}, \xi_{2}\right) / \sqrt{1+\xi_{1}}
\end{aligned}
$$

